University of Louisville

ThinkIR: The University of Louisville's Institutional Repository

Electronic Theses and Dissertations

$12-2014$

\title{
Modulation of host polyubiquitination by the ankb f-box protein of Legionella pneumophila.
}

\author{
William M. Bruckert \\ University of Louisville
}

Follow this and additional works at: https://ir.library.louisville.edu/etd

Part of the Bacteriology Commons, and the Pathogenic Microbiology Commons

\section{Recommended Citation}

Bruckert, William M., "Modulation of host polyubiquitination by the ankb f-box protein of Legionella pneumophila." (2014). Electronic Theses and Dissertations. Paper 1724.

https://doi.org/10.18297/etd/1724

This Doctoral Dissertation is brought to you for free and open access by ThinkIR: The University of Louisville's Institutional Repository. It has been accepted for inclusion in Electronic Theses and Dissertations by an authorized administrator of ThinkIR: The University of Louisville's Institutional Repository. This title appears here courtesy of the author, who has retained all other copyrights. For more information, please contact thinkir@louisville.edu. 
MODULATION OF HOST POLYUBIQUITINATION BY THE

ANKB F-BOX PROTEIN OF LEGIONELLA PNEUMOPHILA

By

\author{
William M Bruckert \\ B.Sc., Middle Tennessee State University, 2009 \\ M.S., University of Louisville, 2011
}

\begin{abstract}
A Dissertation
Submitted to the Faculty of the School of Medicine of the University of Louisville in Partial Fulfillment of the Requirements for the Degree of

Doctor of Philosophy

Department of Microbiology and Immunology

University of Louisville

Louisville, Kentucky
\end{abstract}

December 2014 
Copyright 2014 by William Bruckert

All rights reserved 

MODULATION OF HOST POLYUBIQUITINATION BY THE

ANKB F-BOX PROTEIN OF LEGIONELLA PNEUMOPHILA

By

William M Bruckert

B.Sc., Middle Tennessee State University, 2009

M.S. University of Louisville, 2011

A Dissertation Approved on

December 1, 2014

By the following Dissertation Committee

Dissertation Director. Dr. Yousef Abu-Kwaik

Dr. Don Demuth

Dr. Matthew Lawrenz

Dr. Richard Miller

Dr. Jonathan Warawa 


\section{DEDICATION}

This dissertation is dedicated to my parents

Mr. William Bruckert

And

Mrs. Judy Bruckert

Who have given me every possible opportunity, and without their support this would not have been possible. My parents have been my best friends and role models throughout my life, and they have encouraged me to pursue my dreams, which included completing this $\mathrm{PhD}$. 


\section{ACKNOWLEDGEMENTS}

I would like to first thank my mentor Dr. Yousef Abu-Kwaik for allowing me to perform the research I chose in his laboratory. If it was not for his commitment to me as a student the process would have been much more complicated. Many thanks to my committee members for their advice, encouragement and support during this process. I would also like to leave a whole hearted thank you to Dr. Jim Graham, who has been a great mentor and friend the past 6 years. I would like to thank all members, former and current of the AbuKwaik lab, that have been great peers and friends throughout the process. Thanks to the Department of Microbiology and Immunology for giving me the opportunity to achieve my academic goal. 


\section{ABSTRACT \\ MODULATION OF HOST POLYUBIQUITINATION BY THE ANKB F-BOX PROTEIN \\ OF LEGIONELLA PNEUMOPHILA}

William M Bruckert

December 1, 2014

Legionella pneumophila is a facultative intracellular pathogen that infects a wide array of protozoan hosts and human alveolar macrophages. L. pneumophila is dependent on a functional Dot/Icm type IVB secretion system that translocates bacterial effector proteins into the host cell cytosol. L. pneumophila genomes encode more than 250 effector proteins, many of which inhibit host cellular processes to form a favorable niche termed the Legionella-containing vacuole (LCV). The eukaryotic-like Dot/Icm translocated effector AnkB contains two eukaryotic-like ankyrin protein-protein interacting domains, one eukaryotic-like F- box domain and an eukaryotic C-terminal CaaX motif. Immediately following attachment of extracellular bacteria, AnkB is translocated into the host cell where it is rapidly farnesylated and anchored to the plasma membrane beneath the attached extracellular bacteria. AnkB recruits the host cell SCF1 E3 ubiquitin ligase machinery to the point of attachment and promotes the lysine ${ }^{48}$-linked polyubiquitination of the AnkB substrates. Interestingly, the proteasomal degradation of the lysine ${ }^{48}$-liked polyubiquitinated proteins increases the levels of intracellular free amino acids within 15 minutes of attachment of extracellular bacteria. This early increase in free cellular amino acids is needed to prevent a starvation response and inhibits differentiation into the non-replicative phase which 
facilitates intracellular replication. The polyubiquitinated proteins surrounding the LCV have a wide range of cellular functions, and include the amino acid transporters SLC1A4 and SLC3A2 and the sodium bicarbonate transporter SLC4A7. In addition the ubiquitinated proteome of the WT strain, the LCV contains proteins involved in the immune response, including interferon regulatory factor 7 and Interleukin-1 receptor-associated kinase 1a. The complete LCV proteome of the WT strain as well as the ankB mutant strain contained E2 ubiquitin-conjugation enzymes, E3 ubiquitin ligases and ubiquitin peptidases. Bioinformatic analysis determined the major metabolic networks within the LCV proteome, including the phosphatidylinositol 4,5 diphosphate pathway and multiple amino acid synthesis pathways. These data showed that AnkB is polyubiquitinated on lysine 67 through lysine ${ }^{11}$-linked polyubiquitination. While lysine ${ }^{11}$-linked polyubiquitination has been shown to target the modified protein for proteasomal degradation, stability of AnkB is not affected following ubiquitination. This highlights a novel example of an F-box effector protein that is modified though lysine ${ }^{11}$-linked polyubiquitination. Taken together, AnkB manipulates multiple eukaryotic cellular pathways to enable intra-vacuolar proliferation of $L$. pneumophila. 


\section{TABLE OF CONTENTS}

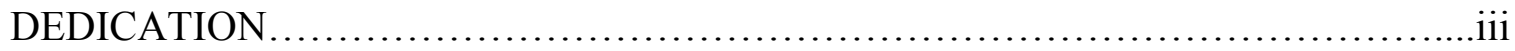

ACKNOWLEDGEMENTS .....................................................

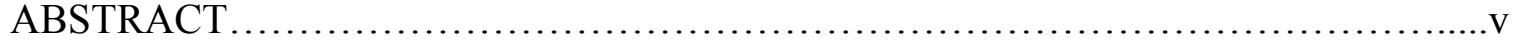

LIST OF FIGURES ..............................................................

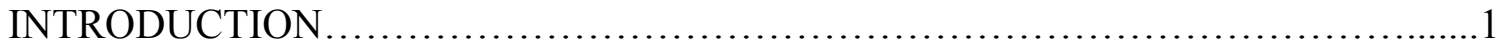

I. Etiology and historical aspects of Legionnaires' disease..$\ldots \ldots \ldots \ldots \ldots \ldots \ldots \ldots \ldots 1$

II. Epidemiology and clinical manifestations of legionellosis.........................2

III. Ecology of L. pneumophila within the aquatic environment......................

IV. Transmission and entry of L. pneumophila to amoeba and macrophages..............4

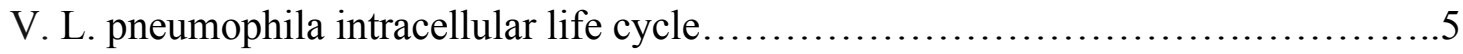

VI. Characterization of the LCV proteome..................................6

VII. L. pneumophila metabolism............................................

VIII. L. pneumophila biphasic life cycle.......................................8

IX. L. pneumophila type II and $1 \mathrm{~V}$ secretion systems...........................

X. L. pneumophila Dot/Icm substrate identification and functions...................11

X1. L. pneumophila eukaryotic-like ankyrin proteins........................... 13

X11. L. pneumophila manipulation of host prenylation machinery.....................15

XIII. Bacterial manipulation of host ubiquitination machinery $\ldots \ldots \ldots \ldots \ldots \ldots \ldots \ldots \ldots \ldots$ 
XIV. L. pneumophila T4SS effector AnkB

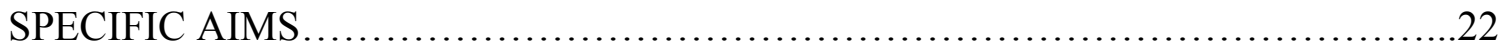

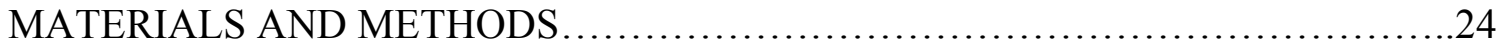

Bacterial strains and cell cultures................................................

Plasmids and DNA manipulations ............................................ 24

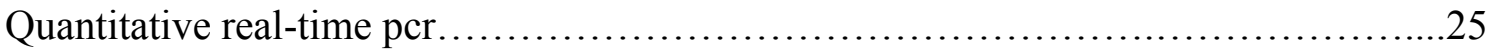

Preferential plasma membrane permeabilization and

loading of the cytosol with antibodies......................................26

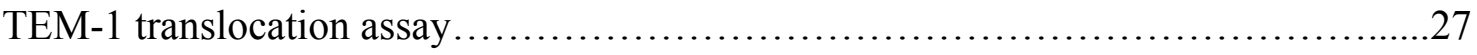

Recruitment of host farnesylation and ubiquitination machinery to

sites of $\mathrm{L}$.pneumophila attachment....................................... 27

Gas chromatography-mass spectrometry analyses

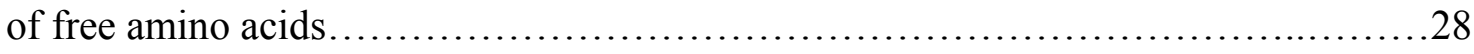

Legionella-containing vacuole purification.................................29

Antibodies and confocal microscopy for LCV

isolation........................................................................

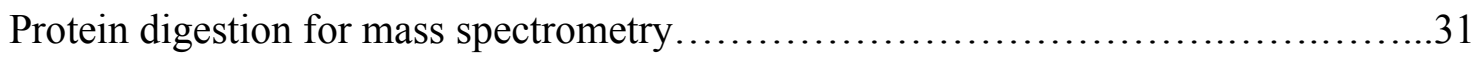

Ubiquitinated protein immunoprecipitation for ubiquitinated LCV

proteome analysis...................................................... 32

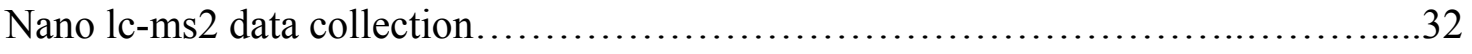

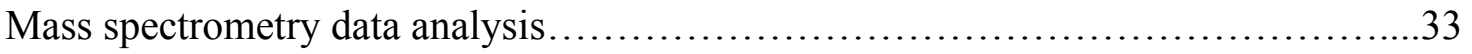

Metacore software analysis.............................................. 34

Transfection of HEK293T cells.................................................. 35 


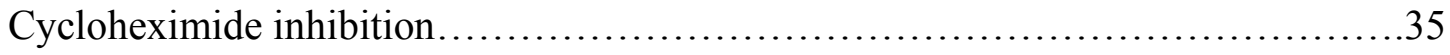

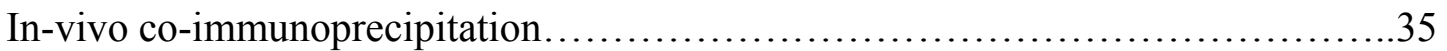

Antibodies and western blot......................................................

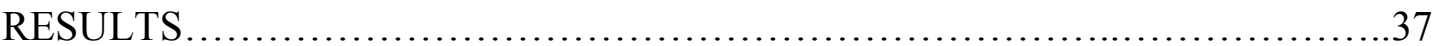

Section 1. Rapid Nutritional Remodeling of Macrophages

Upon Attachment of Legionella pneumophila......................................37

Triggering transcription of ankB upon attachment of L. pneumophila

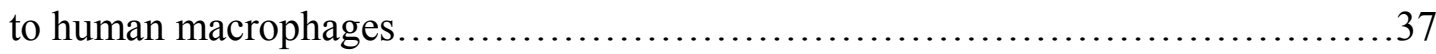

Translocation of AnkB into macrophages upon bacterial attachment...................39

Host-mediated farnesylation and anchoring of AnkB to the cytosolic side of the

plasma membrane beneath attachment sites of extracellular bacteria...................41

Recruitment of the host farnesylation machinery to the plasma membrane

beneath attached extracellular L. pneumophila..................................45

Recruitment of the host SCF1 ubiquitin ligase complex beneath attached

extracellular L. pneumophila...................................................46

Elevated levels of free amino acids following attachment of extracellular

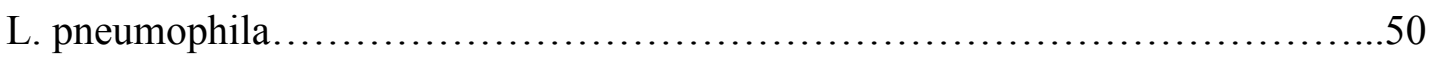

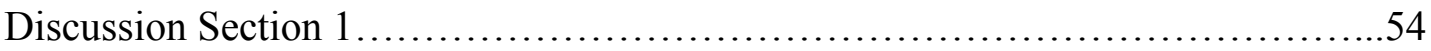

Section 2. The complete and ubiquitinated proteome of the Legionella-containing

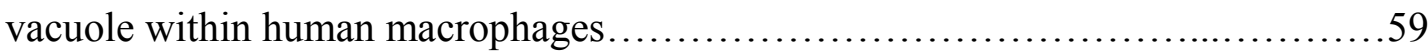

Purification of the LCV from human macrophages................................59

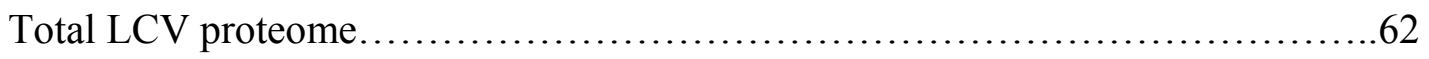

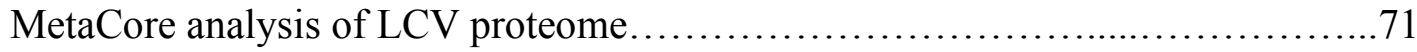


Ubiquitinated proteome of the LCV.

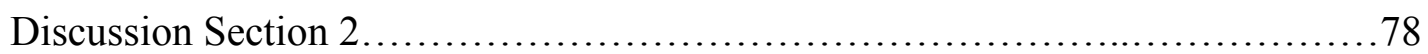

Section 3. Lysine ${ }^{11}$-linked polyubiquitination of the AnkB effector

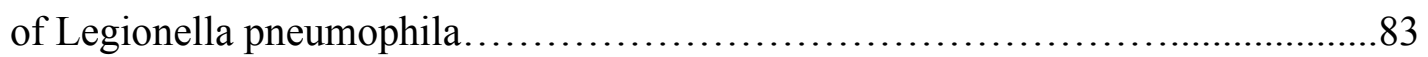

Ubiquitination of the L. pneumophila translocated effector AnkB...............83

Interaction of AnkB with the E3 ubiquitin ligase Trim $21 \ldots \ldots \ldots \ldots \ldots \ldots \ldots \ldots \ldots . \ldots . \ldots . \ldots . \ldots$

Ubiquitinated AnkB is not degraded by the proteasome........................ 88

Polyubiquitin linkages of polyubiquitinated AnkB..............................91

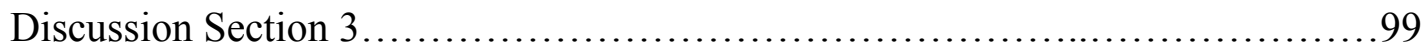

CONCLUSSIONS AND FUTURE DIRECTIONS ............................ 104

REFERENCES..................................................... 108

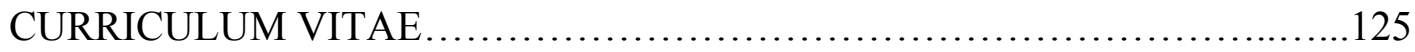




\section{LIST OF FIGURES}

Section 1. Rapid Nutritional Remodeling of Macrophages

Upon Attachment of Legionella pneumophila............................... 37

Figure 1: Figure 1: Expression of ankB upon intimate attachment to hMDMs........39

Figure2: Translocation of BlaM-AnkB fusion protein by L. pneumophila upon

intimate attachment to U937 cells........................................40

Figure 3: Anchoring of native AnkB injected by attached L. pneumophila to the cytosolic side of the plasma membrane of hMDMs beneath bacterial attachment

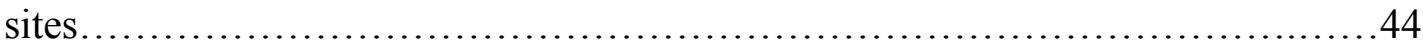

Figure 4: The farnesylation machinery components, FT $\alpha$, RCE1 and IcmT are recruited beneath attachment sites of L. pneumophila to hMDMs.................46

Figure 5: Recruitment of the SCF ubiquitin ligase components Skp1 and Cul1 beneath attachment sites of L. pneumophila to hMDMs and their subsequent

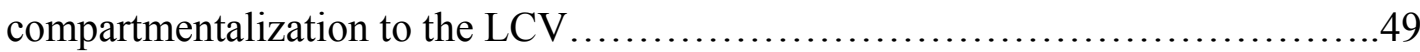

Figure 6: Intimate attachment of L. pneumophila to hMDMs triggers an increase in

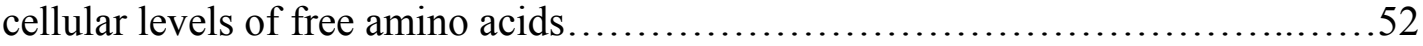

Figure 7: A working model of AnkB-mediated nutritional preparation of the host cell by attached extracellular L. pneumophila................................53

Section 2. The complete and ubiquitinated proteome of the Legionella-containing vacuole within human macrophages 
Figure 8: LCV purification using a discontinuous sucrose gradient...

Figure 9: Functional classification of eukaryotic proteins localized to the

WT strain LCV

Figure 10: Functional classification of eukaryotic proteins localized to the ankB

mutant strain LCV .68

Figure 11: MetaCore enrichment analysis of metabolic networks in the WT strain

LCV proteome .73

Section 3. Lysine ${ }^{11}$-linked polyubiquitination of the AnkB effector

of Legionella pneumophila. 83

Figure 12: Ubiquitination of ectopically expressed L. pneumophila strain AA100/130b and the Paris strain AnkB. .85

Figure 13: Ubiquitination of F-box variants of AnkB ......................... 86

Figure 14: AnkB directly interacts with the E3 ubiquitin ligase Trim $21 \ldots \ldots \ldots . .88$

Figure 15: Ubiquitinated AnkB is not degraded by the proteasome............... 90

Figure 16: AnkB is not polyubiquitinated through K48 or K63 linkages............ 93

Figure 17: Commassie stain of immunoprecipitated AnkB.....................94

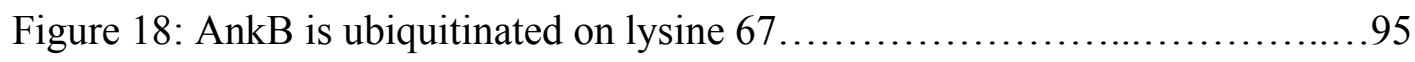

Figure 19: AnkB is polyubiquitinated through lysine ${ }^{11}$ of ubiquitin................96

Figure 20. Substitution of AnkB K ${ }^{67}$ does not inhibit polyubiquitination ...........98 


\section{INTRODUCTION}

\section{Etiology and historical aspects of Legionnaires' disease}

Legionnaires' disease was first described in 1976 following an outbreak of pneumonia at the American Legion Convention in Philadelphia, Pennsylvania, which included 34 death $[1,2]$. The causative agent was determined to be a facultative intracellular Gram-negative bacterium, assigned the name Legionella pneumophila and the strain was designated Philadelphia-1 [1]. L. pneumophila belongs to the Legionellaceae family in the gamme-2 subdivision of the Proteobacteria, in which the Legionellales order has been created [3-5]. The Legionellales order contains two families: Legionellaceae and Coxiellaceae, each having the characteristics of being intracellular parasites infecting humans, animals and protozoa [6]. Within the Legionellaceae family there are $\sim 60$ species and 72 serogroups of bacteria which have been isolated from clinical and environmental conditions $[7,8]$. L. pneumophila consists of 16 serogroups, of which serogroup 1 is the most common in infection, followed by 4 and $6[9,10]$. In the United States L. pneumophila represents more than $90 \%$ of community or hospital acquired Legionnaires' disease cases, with serogroup 1 responsible for approximately 70\% [10]. However, in Western Australia and New Zealand L. longbeachae accounts for 30.4\% of community acquired Legionnaires' disease cases $[11,12]$. 


\section{Epidemiology and clinical manifestations of legionellosis}

L. pneumophila is capable of causing Legionnaires' disease and Pontiac fever, both known as legionellosis. Humans acquire legionellosis through the inhalation of L.pneumophila containing aerosols, primarily from mechanical means such as showers, water fountains, spas and air conditioning towers [13-15]. Following an incubation period of 2-10 days the initial clinical symptoms of Legionnaires' disease, often nonspecific, include mild cold symptoms, malaise, low fever, headache, anorexia and muscle aches $[8,16,17]$. Without treatment, symptoms progress to a high fever accompanied by a life threatening pneumonia, fibrinolysis, cellular infiltrations of macrophages and neutrophils, and alveolar damage $[8,18,19]$. Pontiac fever is an acute, self-limiting, non fatal respiratory infection without pneumonia [20]. The symptoms of Pontiac Fever, which mimic influenza infection, are fever, chills, sore throat, abdominal pain and a nonproductive cough [20-22]. The immune system of the host is critical for L. pneumophila infection, as healthy individuals typically clear the infection, individuals with chronic lung diseases, diabetes or chronic renal failure have a high risk of disease development if infected [10, 23]. Annually there are an estimated 25,000 worldwide cases of pneumonia caused by L. pneumophila, with approximately 10,000 cases in the USA according to the Center of Disease Control and Prevention, with a mortality rate ranging from 7-24\% [24]. However, it is assumed that only 2-10\% of Legionnaires disease cases are reported [25]. Nosocomial L. pneumophila infections have a fatality rate reaching $50 \%$ and are associated with recent surgeries, mechanical ventilation, and transplant procedures such as heart and liver [26-28]. Legionnaires' disease is always transmitted from the environment to humans; therefore, 
environmental monitoring of cooling towers and related water sources should be routinely surveyed to control the spread of the disease.

\section{Ecology of $L$. pneumophila within the aquatic environment.}

L. pneumophila is ubiquitous in natural water sources such as rivers and lakes, as well as manmade water systems such as air conditioning units, water fountains and cooling towers $[13,14,29,30]$. L. pneumophila and other bacterial species form biofilms in the aquatic environment, which have been shown to increase their resistance to microbial disinfection techniques such as chlorination and UV treatment $[31,32]$. Within aquatic environments $L$. pneumophila invade and replicate within many species of protozoa, which play a pivotal role in the amplification of the organism as well as providing an intracellular habitat capable of providing adequate nutrients and protecting the bacteria from high temperature, drying, and chlorination of the extracellular environment $[33,34]$.

L. penumophila grown in amoeba have a greatly increased resistance to the antimicrobial properties of the biocides polyhexamethylene biguanide (PHMB) and benzisothiazolone (BIT), and are 1,000 fold more resistant to the antimicrobial effects of rifampin and ciproflaxin $[35,36]$. Furthermore, L. pneumophila grown in amoeba are 100 and 10 fold more invasive for epithelial cells and macrophages, respectively [37]. This increase in infectivity is associated with changes in bacterial morphology, cell wall composition and structure, and the route of entry into macrophages [34, 38, 39]. 


\section{Transmission and entry of $L$. pneumophila to amoeba and macrophages}

Inhalation of aerosolized water droplets containing infectious $L$. pneumophila can occur through multiple routes following the bacteria-protozoan interaction. Following escape from the protozoan host or biofilm, infectious extracellular L. pneumophila can be transmitted to humans through a contaminated water source. Other possible routes of infection include the inhalation of excreted L. pneumophila-filled vesicles or amoeba filled with $L$. pneumophila $[40,41]$. L. pneumophila uptake into macrophages is mediated by human monocyte complement receptors CR1 and CR3 which trigger microfilamentdependent phagocytosis, while L. pneumophila uptake by amoeba is microfilamentindependent [42-44]. L. pneumophila attachment to the protozoan host Hartmannella vermiformis is associated with a $170-\mathrm{kD}$ galactose/ $N$-acetyl-D-galactosamine (Gal/ GalNAc) inhibitable lectin and subsequent tyrosine dephosphorylation of multiple host cytoskeletal proteins [45-47]. However, in Acanthamoeba polyphaga bacterial attachment is not associated with the galactose/ $N$-acetyl-D-galactosamine (Gal/ GalNAc) inhibitable lectin, and there is only a slight tyrosine dephosphorylation of a single host protein [45]. Uptake into $H$. vermiformis occurs mainly through cup-shaped invaginations (zipper phagocytosis), while coiling phagocytosis is used by Acanthamoeba castellanii and human macrophages [37, 4749]. Thus, $L$. pneumophila has evolved diverse mechanisms to invade different species of amoeba and human macrophages by using different ligands and receptors on the host cell for attachment. 


\section{L. pneumophila intracellular life cycle}

In contrast to the diverse mechanisms of attachment and uptake of L. pneumophila by protozoa and human cells, intracellular trafficking and survival techniques of the bacterium are indistinguishable between the two evolutionary distant hosts [50-52]. Upon inhalation of infectious L. pneumophila water droplets, human alveolar macrophages use coiling phagocytosis to ingest the bacterium $[53,54]$. Following entry, L. pneumophila intercepts ER-to-Golgi vesicular traffic and resides in a membrane-bound vacuole (Legionellacontaining vacuole) that becomes surrounded with endoplasmic reticulum derived smooth vesicles, mitochondria and other host cell organelles within five minutes $[51,55,56]$. Within 4 hours following infection the smooth vesicles on the Legionella containing vacuole (LCV) are replaced with a ribosome studded multilayer membrane derived from the rough endoplasmic reticulum $[48,52,57]$. Importantly, wild type L. pneumophila evades phagosome-lysosome fusion in human macrophages or amoeba as seen by the absence of the lysosomal markers LAMP-1, LAMP-2 and cathepsin D on the LCV [48, 58-60]. L. pneumophila begins multiplying after a four hour log phase, and continues until the LCV membrane ruptures, releasing bacteria into the cytosol where two-three rounds of additional replication occur $[13,61]$. Eventually the lack of nutrients causes a starvation response and differentiation into the post exponential phase, resulting in the bacteria lysing the macrophage and release of bacteria into the extracellular environment to infect a new host $[51,52]$. During infection of macrophages, L. pneumophila causes activation of caspase-3 throughout the infection, however, apoptotic cell death is not initiated until late stages of the infection [62-66]. L. pneumophila prevents premature caspase-3 induced apoptotic cell death by triggering anti-apoptotic signaling through NF-kappaB activation and inhibition of the 
type I interferon response [67-69]. L. pneumophila has been shown to kill human macrophages and A. polyphaga through temporal induction of necrosis, mediated by the pore-forming toxin activity of $L$. pneumophila upon termination of intracellular replication $[61,70,71]$. Mutants defective in pore-forming toxin activity replicate to the same extent as the wild type strain, however, they fail to lyse the host cell and remain trapped within the LCV [61, 72]. Therefore, intracellular replication and phagosomal remodeling through interception of host cell vesicular trafficking is independent of host cell lysis.

\section{Characterization of the LCV proteome}

Using the JR32 Philadelphia-derived strain of L. pneumophila the LCV proteome has been profiled from RAW264.7 mouse macrophages [73, 74]. The LCV proteome has also been generated using the Corby strain of L. pneumophila in the amoeba host Dictyostelium discoideum [73-75]. While the LCV proteome from RAW264.7 macrophages presents information on LCV biogenesis, RAW264.7 macrophages originate from the BALB/C mouse, whose primary macrophages are non-permissive to L. pneumophila infection [76, 77]. The LCV proteome analysis from Dictyostelium discoideum contained 566 host proteins [73], while the LCV proteome from RAW264.7 macrophages contained 1156 proteins [74]. A large proportion (>50\%) of the identified proteins were associated with cellular metabolism, while proteins involved in signaling and trafficking were found in lower abundance ( 10\% each) [74]. The LCV recruits small GTPases, such as Rab1, Sar1 and ADP ribosylation factor 1, which are critical for ER-to-Golgi trafficking [56, 78, 79]. In addition, 14 other Rab GTPases were identified in the proteome which include Rab2, Rab4, Rab5, Rab7, Rab8 [74]. These Rab GTPases indicate the fusion of the LCV with early and 
late endosomes and distinct vesicle trafficking pathways in the host cell. The LCV is decorated with phospatidylinositol-4 phosphate (PI(4)P), which multiple Dot/Icm bacterial secreted effector proteins (SidC, SidM, SdcA, Rid) bind on the LCV membrane [80-82]. While there were 60 Dot/Icm effector proteins identified in the RAW264.7 LCV proteome analysis, some of these effectors have transmembrane domains which could allow them to localize to the LCV (MavP, MavE), it is unknown how the other effector proteins anchor into the LCV $[74,83]$. Post-translational modifications, such as farnesylation or ubiquitination, of effector proteins could alter their sub-cellular localization and allow the effectors to localize with the LCV, or with vesicles that fuse with the LCV during vacuole biogenesis. The effectors on the LCV could interact with specific host proteins critical for intracellular survival.

\section{L. pneumophila metabolism}

L. pneumophila obtains carbon and energy from amino acids, primarily through the TCA cycle, with Ser, Glu, Tyr and Thr used for growth in vitro, and Cys, Gln, Ser, and Arg supporting growth in vivo [84, 85]. L. pneumophila is auxotrophic for 7 amino acids (Cys, Met, Arg, Thr, Val, Ile and Leu), and expresses $~ 12$ classes of ATP-binding cassette transporters [86] and amino acid permeases encoded in the genome [87, 88]. Intracellular replication of L. pneumophila is dependent upon the host cell amino acid transporter SLC1A5 during intracellular infection of human macrophages [85, 89]. With L. pneumophila having a doubling time of $\sim 40$ minutes in macrophages and amoeba, acquisition of host amino acids is critical to provide the essential nutrients to support the energy spent during intracellular survival and proliferation [90]. It has been shown that L. pneumophila 
incorporates host cell amino acids during infection of Acanthamoeba castelannii,[91].

Therefore it is likely that host amino acid transporters are localized to the LCV; however, this has not been evaluated. While amino acids are the major source of carbon and energy, $L$. pneumophila can convert exogenous glucose to pyruvate through the Entner-Doudoroff pathway [88, 92]. The Entner-Doudoroff pathway plays an important role in the infection process since Entner-Doudoroff pathway mutants have a significant growth defect in Acanthamoeba culbertstoni, A/J mouse macrophages and A549 human epithelial cells [92]. Interestingly, the $a n k B$ mutant which is defective for intracellular replication due to a lack of amino acids, can be rescued for growth with the addition of pyruvate to the growth medium [86]. Therefore, intracellular replication of L. pneumophila is dependent upon more than amino acids, and is a combination of other carbon and energy sources, although amino acids are the major source of carbon and energy.

\section{L. pneumophila biphasic life cycle}

In natural environments, $L$. pneumophila alternates between a replicative phase and a highly infectious nonreplicative transmissive phase [34, 93]. As vacuole nutrients become limiting during late stages of intra-vacuolar proliferation, exponential phase bacteria respond by producing the alarmone ppGpp, which stimulates signaling pathways responsible for the differentiation and phenotypic phase variation seen upon entering the post-phase [94, 95]. The ppGpp synthases RelA and SpoT are essential for phenotypic variation and become activated during nutrient limitation at the end of intracellular replication, which causes an increase in the alarmone ppGpp [94-96]. In response to the increase of ppGpp the sigma factor RpoS and the LetA/S two component system coordinate the activation of many 
transmission virulence traits (cytotoxicity, flagellum synthesis, increased osmotic resistance, increased resistance to extracellular stresses, and evasion of the endocytic pathway). These transmission virulence traits are not expressed by exponential replicating bacteria, and are thought to be required for escaping a nutrient-deprived host, gaining entry into the next host, and suppressing traits dedicated to intracellular replication [94-96]. The repressor of transmission traits, CsrA, is active exclusively during the replicative phase while lysosomal evasion traits are inactive during intracellular replication [60, 97]. The flagella sigma factor FliA, which activates several genes needed for flagellum development, is required for the post-exponential traits of motility, cytotoxicity, infectivity and lysosome evasion [98, 99]. Genes regulated by FliA could explain how L. pneumophila can exit one host and block phagolysosomal fusion in the next.

\section{L. pneumophila type II and 1V secretion systems}

Gram-negative bacteria have developed sophisticated secretion systems in order to deliver effector molecules from the bacteria cell to the cytoplasm of the host cell or to the extracellular environment. Of the eight well characterized secretion systems in Gramnegative bacteria, L. pneumophila contains a functional type II (Lsp) and type IV (Dot/Icm) secretion system [100-103]. The L. pneumophila type II secretion system involves the Sec or Tat pathway, which secretes proteins across the bacterial inner membrane to the periplasm, where proteins are unfolded and secreted across the outer bacterial membrane through the type II secretion system complex $[104,105]$. The L. pneumophila type II secretion system is responsible for the secretion of many degradative enzymes such as acid phosphatases, lipases, aminopeptidases, and a zinc metalloprotease [106-109]. L. pneumophila type II 
secretion is required for the intracellular infection of Acanthamoebae and Hartmannellae, optimal intracellular infection of human macrophages, and virulence in the murine model $[100,110,111]$.

The Dot/Icm Type IVB secretion system was coincidentally discovered by 2 groups who sought to determine L. pneumophila virulence traits by generating spontaneous mutants and assessing their ability to replicate within macrophages, and to avoid phagosomelysosome fusion $[112,113]$. The L. pneumophila spontaneous avirulent strain 25D was restored for intracellular replication when a region of the chromosome (intracellular multiplication (Icm)) was complemented with the wild type strain region of the chromosome [113-115]. Subsequently, a second region of the genome (defect in organelle trafficking (Dot)) was able to restore phagosome-lysosome evasion and association with host cell organelles in an avirulent strain $[112,116]$. The Dot/Icm loci are composed of 26 genes responsible for the assembly of a sophisticated type IVB secretion system [103, 117, 118]. Type IV secretion systems (T4SS) are macromolecular systems with homology to the conjugation machinery required for the conjugation of plasmids between bacteria $[103,119$, 120]. The Dot/Icm secretion system is made up of 22 structural proteins, many of which are membrane associated, and 5 chaperone proteins that interact with effector proteins in the bacteria cytoplasm [121, 122]. In contrast to the Lsp Type II secretion system which secretes approximately 20 proteins, primarily degradation enzymes to the extracellular environment, the Dot/Icm secretion system translocates more than 250 bacterial proteins directly into the host cell cytoplasm [123]. L. pneumophila is naturally competent in the environment, therefore the ability to transfer the Dot/Icm effector proteins from one bacterium to another could account for this extraordinary number of effectors compared to other bacteria [124]. 
Despite the requirement for a functional type IV secretion system for intracellular replication and phagosome-lysosome evasion, loss of single effectors rarely cause a significant growth defect $[125,126]$. This is thought to primarily result from redundancy among the effector proteins, however single deletion mutants of many effectors have not been analyzed [125].

\section{L. pneumophila Dot/Icm substrate identification and functions}

The requirement of the Dot/Icm secretion system for intracellular infection has lead research into the identification and characterization of the translocated substrates. Techniques utilized to identify L. pneumophila translocated effectors include genetic assays in yeast, proteins interacting with Dot/Icm components, bioinformatic searches for genes encoding eukaryotic domains, and direct translocation assays looking for the presence of the effector protein within host cells [123, 127-130]. In addition, L. pneumophila effector proteins were identified by using the T4SS chaperone-like IcmW protein as bait in a yeast two-hybrid screen [127]. Translocation has been determined using fusion reporters such as the adenylate cyclase and beta-lactamase assays, where the bacterial gene of interest is fused to the Bordetella pertussis adenylate cyclase catalytic domain, or the carboxyl end of TEM-1 betalactamase, respectively $[123,131,132]$. The calmodulin-dependent adenylate cyclase assay causes a dramatic increase in intracellular cyclicAMP levels when the fusion protein is translocated into host cells [133]. In the fluorescent resosnance energy transfer (FRET) assay based on TEM-1 $\beta$-lactamase activity, the host cells are loaded with a $\beta$-lactamase substrate (CCF4-AM), which emits green fluorescence $(520 \mathrm{~nm})$. If the effector protein containing the TEM-1 fusion reporter is translocated into loaded cells, the substrate will be cleaved and emit blue fluorescence $(447 \mathrm{~nm})$, allowing translocation to be determined by measuring the ratio of 
cleaved to uncleaved substrate in infected cells. Antibodies specific for Dot/Icm substrate proteins have been utilized in confocal microscopy to determine sub-cellular localization in the host cell [124, 134-136].

Sequence analysis of L. pneumophila genomes identified many of the predicted and experimentally verified Dot/Icm substrates are similar to eukaryotic proteins or have motifs commonly found in eukaryotic proteins $[137,138]$. These effectors are thought to have been acquired through horizontal gene transfer during the evolution of $L$. pneumophila with primitive eukaryotic host cells such as amoeba $[137,138]$. L. pneumophila encodes effectors with eukaryotic like motifs such as Set domains, ankyrin domains, U-box and F-box motifs, and eukaryotic like proteins such as sphingosine kinase and phosphatases [139, 140]. Many of the eukaryotic like proteins interfere with host cell pathways contributing to intracellular trafficking, and manipulation of host cell processes that are advantageous to intracellular bacterial survival [141, 142].

Despite the requirement for a functional type IV secretion system for intracellular replication and phagosome-lysosome evasion, loss of a single effector rarely causes a significant defect in intracellular replication $[125,126]$. The effector protein DrrA (SidM) is necessary for host cell Rab1 recruitment to the LCV, while the effector protein LidA enhances this Rab1 recruitment, showing a specific redundancy in the functions of different effector proteins [143-145]. The host guanosine triphosphatase (GTPase) Rab1 is recruited to the LCV within 5 minutes of uptake, and is involved in the recruitment of ER-derived vesicles to the $L C V$ to create a replicative niche $[78,146,147]$. A lidA mutant of $L$. pneumophila strain has a slight growth defect in human macrophages, while intracellular replication of a $\operatorname{Drr}$ (sidM) mutant strain was similar to wild-type L. pneumophila [134, 
143]. The L. pneumophila effector protein SdhA has been shown to be involved in maintaining the integrity of the LCV and preventing host cell death through suppression of type 1 interferon, while the effector SidF also inhibits host cell death by inhibiting proapoptotic signaling through Bcl2 $[69,148,149]$. Interestingly, a L. pneumophila sdhA mutant strain was severely impaired for intracellular growth within A/J mouse macrophages due to caspase-1 dependent host cell death [150, 151]. However, in U937 macrophages and D. discoideum intracellular replication was only partially impaired in the $s d h A$ mutant strain [150]. In contrast to the effectors causing anti-apoptotic signaling at least five effectors of $L$. pneumophila are capable of causing caspase 3 activation [152]. Inhibiting host protein translation is one of the main mechanisms of $L$. pneumophila effectors [153, 154]. At least 5 effectors (Lgt1, Lgt2, Lgt3, SidI, SidL) inhibit host protein translation by modifying the host elongation factor eEF1A or by binding the host elongation factor EF1B $\gamma$, which blocks protein translation $[153,155]$. Importantly, the effectors that block translation lead to an exasperated Map kinase response where host transcribed mRNA fail to be translated [155]. During L. pneumophila induced translation inhibition, selected genes such as IL-1 $\alpha$ and IL$1 \beta$ are still translated, which is likely due to them being the most abundant transcripts during L. pneumophila infection $[155,156]$. These examples highlight the ability of $L$. pneumophila to manipulate a wide variety of host cellular pathways to obtain a favorable intracellular environment during infection.

\section{L. pneumophila eukaryotic-like ankyrin proteins}

Through bioinformatic analysis of L. pneumophila genomes, many eukaryotic-like proteins have been identified, which are thought to have been acquired through horizontal 
gene transfer $[129,137,138,142]$. Eukaryotic proteins containing ankyrin domains are broadly distributed in the cytoplasm and the nucleus and act as adaptors to mediate specific protein-protein interactions $[157,158]$. The 33 amino acid ankyrin repeat (ANK) is the most prevalent motif found in protein databases [159-163]. Eukaryotes contain the majority of ankyrin repeat proteins, while bacteria, archaea and viruses have ankyrin repeat proteins as well [159]. Eukaryotic ankyrin proteins are involved in development, cell signaling, inflammatory response, inhibition or development of tumors, cell-cycle regulation and signal transduction [164-166]. The ankyrin motif exhibits helix-turn-helix conformation, with the beta loop projected outward to bind the target protein [167-169]. The ANK repeat binding site consists of six non-conserved amino acid residues, which determine the specific target of the ankyrin protein $[170,171]$. Ankyrin proteins have been identified in many different bacterial pathogens such as Wolbachia, Pseudomonas, Coxiella, Anaplasma and Legionella [129, 172-175]. Coxiella burnetti translocated ankyrin proteins localize to different host cell organelles, where they are hypothesized to manipulate host cell processes such as apoptosis and vesicular fusion events critical for infection [174].

L. pneumophila strains Corby, Lens, Paris and Philadelphia contain at least eleven common ankyrin genes (ankB, ankC, ankD, ankE, ankF, ankG, ankH, ankI, ankJ, ankK, $\operatorname{ankN})$, which exhibit extensive size and sequence variation $[129,159]$. The ankyrin proteins in L. pneumophila vary extensively, such as containing between 1-11 ANK repeats, one Fbox domain, and one SET motif [129]. Adenylate cyclase translocation assays revealed at least eight of the ankyrin proteins are translocated into the host cell through the Dot/Icm T4SS [176]. Most of the ankyrin genes are upregulated in vitro and within A. polyphaga during the transition to the post-exponential phase in an RpoS-dependent mechanism [129, 
177]. While eight of the ankyrin genes are dispensable for intracellular growth, AnkH, AnkJ, and AnkB play crucial roles in the infection of hMDMs and multiple protozoan hosts [129, 131]. The L. pneumophila ankH and ankJ mutant strains exhibit a major defect in intracellular replication within human macrophages, a partial defect within A. polyphaga and H.vermiformis, and are attenuated in the mouse model of Legionnaires' disease $[129,176]$. The ankyrin domains and the last ten C-terminal amino acid residues of AnkH and AnkJ are required for translocation and proficient intracellular replication [176]. The ankH and ankJ mutant strains exhibited similar intracellular trafficking as the wild-type strain, indicating these effector proteins are not involved in vacuole formation or trafficking, but are needed to promote intracellular replication within the LCV [176].

\section{L. pneumophila manipulation of host prenylation machinery}

Prenylation is an irreversible post-translational lipid modification of a protein, involving the covalent addition of a 20-carbon geranygeranyl group or a 15-carbon farnesyl isoprenoid group, to a cysteine residue within the conserved C-terminal CaaX motif (in which "a" is any aliphatic amino acid and " $X$ " is any amino acid) $[178,179]$. This modification increases protein hydrophobicity, which results in anchoring of the protein to the lipid bilayer of cell membranes or organelle membranes $[180,181]$. In humans three different protein prenyltransferases facilitate protein prenylation: two geranylgeranyl protein transferases (GGtase I and II) and one farnesyltransferase (FTase) [182, 183]. Following prenylation of the cysteine, the terminal "aaX" tripeptide is cleaved by RAS-converting enzyme 1 (RCE-1), and the prenylcysteine is carboxymethylated by isoprenyl cysteine carboxyl methyl transferase (ICMT) $[184,185]$. Labeling experiments have estimated that $2 \%$ of the 
mammalian proteome is prenylated, and the characterized farnesylated or geranylgeranylated proteins include kinases, phosphatases, GTP-binding proteins and nuclear lamins [186, 187]. The most characterized CaaX motif proteins are Ras GTPases and trimeric G proteins, which require an additional signal to target specific cellular compartments $[188,189]$. The signal in Ras proteins is located immediately upstream from the CaaX motif known as the hypervariable domain, while trimeric $\mathrm{G}$ proteins must become modified through the attachment of the fatty acid palmitate or myristate on the $\alpha$ subunit $[190,191]$. CaaX motifcontaining proteins are involved in cellular signaling processes and regulatory events including cell proliferation, differentiation, metabolism, apoptosis and nuclear stability [192194].

Genomic analyses of 17 intracellular and extracellular pathogens predicted 54 proteins from 14 species, including Salmonella, Legionella, Yersinia, Francisella among others, have the C-terminal CaaX motif that could be subjected to host prenylation [195, 196]. The Salmonella enterica T3SS effector protein SifA is geranylgeranylated, resulting in localization to the Salmonella containing vacuole (SCV) and Salmonella-induced filaments, where it interacts with host cell Rab7, and plays a role in preventing Salmonella-containing vacuole-lysosome fusion $[197,198]$. In the L. pneumophila strains Philadelphia, Lens, Corby, and Paris eleven CaaX motif-containing proteins (CMPs) were identified, with six of the proteins being conserved among the sequenced L. pneumophila strains [199, 200]. The adenylate cyclase translocation assay identified seven of the Legionella CMPs are translocated into the host cell through the Dot/Icm T4SS [199, 200]. Legionella CMPs are substrates of eukaryotic prenylatransferases, causing lipidation and enabling their targeting to specific cellular membranes [199]. When ectopically expressed in human cells, Legionella 
CMPs localize as intense punctuate spots or around the cell periphery, indicating vesicular or plasma membrane localization, respectively [199]. Inhibition of prenylatransferases cause a significant but modest increase in wild-type LCV's acquiring the lysosomal markers LAMP1 and Cathepsin D [199]. Therefore, manipulation of host cell prenylation machinery through L. pneumophila CMPs contributes to evasion of phagosome-lysosome fusion and enhances the ability of remodeling of the LCV.

\section{Bacterial manipulation of host ubiquitination machinery}

Posttranslational modifications of proteins are conserved mechanisms for regulating protein activity, localization, conformation, or stability. Protein modifications, usually driven by enzymes, include phosphorylation, lipidation, protonation, ubiquitination, and prenylation. Many pathogenic bacteria use translocated effectors to manipulate host cell posttranslational machineries to promote intracellular bacterial replication and survival.

Ubiquitination is a conserved eukaryotic post-translational protein modification involving the addition of a 76 amino acid ubiquitin moiety onto a substrate protein via isopeptide bond between free carboxy group on the terminal glycine of ubiquitin and the $\varepsilon$ amino on lysine of the target protein [201, 202]. Polyubiquitination is the covalent linkage of ubiquitin monomers through 1 of 7 lysine residues in ubiquitin (K6, K11, K27, K29, K33, K48, K63) [202, 203]. The fate of the polyubiquitinated protein depends on the lysine residue within ubiquitin that is involved in the ubiquitin-ubiquitin chain linkage [203, 204]. Polyubiquitination through $\mathrm{K}^{63}$ regulates nonproteolytic cellular processes, such as 'transcriptional activation, protein localization, innate immune response, cell cycle progression and DNA damage response [205-208]. If ubiquitin monomers are linked through 
$\mathrm{K}^{48}$ residues, the substrate protein is recognized by the $26 \mathrm{~S}$ proteasome and degraded [209, 210]. While polyubiquitination through $\mathrm{K}^{6}, \mathrm{~K}^{11}, \mathrm{~K}^{27}, \mathrm{~K}^{29}$ and $\mathrm{K}^{33}$ is less studied, however with new techniques it is becoming more common to observe these types of polyubiquitination, it has been shown these linkage patterns can cause substrate degradation, endocytosis, signaling and regulation [211-213]. Polyubiquitination occurs through a threestep process involving activation of ubiquitin by an E1 enzyme [214], allowing for transfer of the ubiquitin moiety to a ubiquitin-conjugating enzyme (E2) [215], followed by transfer onto the targeted protein via substrate specific ubiquitin-protein ligases (E3) [216]. There are $>1,000$ estimated substrate-specific ubiquitin ligases, classified into two major groups, RING-type and HECT-type [216, 217]. The SKP1-CUL1-F-box (SCF) complex is a well characterized RING-type E3 ubiquitin ligase complex [218]. The E2 ubiquitin conjugating enzyme binds the RBX1 RING finger protein, which associates with the $\mathrm{C}$ terminus of the cullin (CUL1) [215]. The F-box domain of the F-box protein binds directly to SKP1, which links the F-box protein to CUL1 to cause ubiquitination of the substrate protein that is bound to another domain of the F-box protein [219, 220]. F-box containing proteins generally have a recognizable substrate protein binding domain, such as a WD40 or a leucine rich repeat, which bind specific substrates to be ubiquitinated [221, 222].

Bioinformatic analyses have revealed eukaryotic-like F-box proteins in human pathogens, plant pathogens, viruses, and amoebal endosymbionts [223-225]. The plant pathogen Agrobacterium encodes 694 potential F-box proteins, while there have been $\sim 70 \mathrm{~F}$ box proteins identified in humans [226, 227]. L. pneumophila encodes five F-box proteins (AnkB, LegU1, LicA, Lpg2224, Lpg2525) that are translocated into host cells through the Dot/Icm T4SS [228]. LegU1 and AnkB associate with the SKP1 and CUL1 components of 
the SCF E3 ubiquitin ligase complex, while LicA only interacts with SKP1 [228]. LegU1 specifically interacts with and causes ubiquitination of the host cell chaperone protein BAT3 [228]. In BAT3 knockout mammalian cells there is an increased resistant to ER stress, therefore, multiple L. pneumophila F-box proteins could target BAT3 for proteasomal degradation to modulate the host ER stress response [228] The L. pneumophila Dot/Icm translocated effector LubX contains 2 domains very similar to the U-box, a domain found in eukaryotic E3 ubiquitin ligases [229]. LubX functions as an E3 ubiquitin ligase, causing polyubiquitination and proteasomal degradation of the L. pneumophila effector protein SidH [230]. A number of bacterial pathogens such as Yersinia, Salmonella and Pseudomonas have effector proteins that manipulate eukaryotic ubiquitination machinery [231]. The Salmonella effector SopA is ubiquitinated in a proteasomal dependent manner [232, 233], while SopB is ubiquitinated through $\mathrm{K}^{63}$-linked polyubiquitination which alters its subcellular localization [234]. The Pseudomonas aeruginosa effector ExoU could be modified through a diubiquitin chain following translocation $[235,236]$. To date no bacterial effector protein has been found to be modified through $\mathrm{K}^{6}, \mathrm{~K}^{11}, \mathrm{~K}^{27}, \mathrm{~K}^{29}$ or $\mathrm{K}^{33}$-linked polyubiquitination. Interestingly, intracellular replication by L. pneumophila is dependent upon $\mathrm{K}^{48}$-linked polyubiquitination of the LCV in a proteasome-dependent mechanism [237, 238]. Therefore, L. pneumophila utilizes acquired eukaryotic-like F-box proteins to manipulate the host cell ubiquitination machinery for regulation of bacterial effector proteins and host cell targets. 


\section{L. pneumophila T4SS effector AnkB}

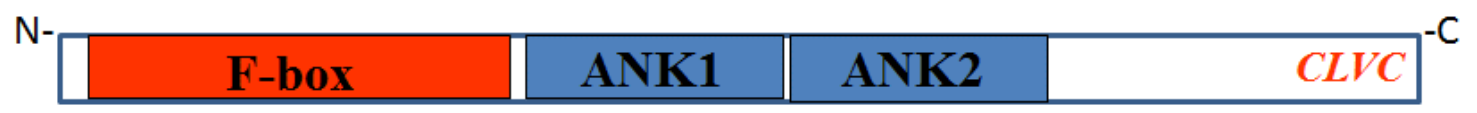

\section{Figure 1. A model of the domain structure of AnkB. AnkB is a eukaryotic-like F-box protein that contains 2 ankyrin domains. The $C$-terminus of AnkB contains a eukaryotic CaaX motif (CLVC).}

The L. pneumophila ankB gene, conserved among all sequenced strains, encodes a 172 amino acid protein that contains two eukaryotic-like ankyrin domains and a conserved $\mathrm{N}$ terminus eukaryotic-like F-box domain $[129,131]$. In the Paris strain and the AA100/130b strain of L. pneumophila, a mutation in ankB causes a severe defect in intracellular replication in human macrophage and $A$. polyphaga $[131,238,239]$. However, in the Philadelphia-derived Lp02 strain, a mutation in ankB does not result in a growth defect within human macrophages or amoeba [228]. In the AA100/130b strain, in-frame deletions of single ankyrin domains in the $a n k B$ gene result in a partial defect in intracellular replication, while a deletion in both ankyrin domains abolishes intracellular replication identical to the $a n k B$ null mutant [240]. Upon extracellular bacterial attachment, AnkB is translocated and triggers assembly of polyubiquitinated proteins directly beneath bacterial attachment site [238]. The C-terminal CaaX motif of AnkB is prenylated though the addition of a farnesyl group, enabling AnkB to anchor into the plasma membrane beneath bacterial attachment and subsequently to the cytosolic face of the LCV membrane [135]. Through conserved residues in the F-box domain, AnkB directly interacts with the SKP1 component 
of the SCF1 E3 ubiquitin ligase complex to recruit $\mathrm{K}^{48}$-linked polyubiquitinated proteins to the LCV [241]. The $\mathrm{K}^{48}$-linked polyubiquitinated proteins are degraded by the proteasomes to generate a surplus of free cellular amino acids that are utilized by the bacterium to provide the carbon and energy for intracellular replication [86]. When macrophages are supplemented with an amino acid mixture, the ankB mutant strain is rescued for intracellular growth, suggesting that the function of AnkB is to provide amino acids as nutrients for intra-vacuolar bacteria [86]. Therefore, AnkB exploits eukaryotic cellular processes to trigger $\mathrm{K}^{48}$-linked polyubiquitination on the LCV. The polyubiquitinated substrates are degraded by the $26 \mathrm{~S}$ proteasome to generate free cellular amino acids that are imported into the LCV for use as a carbon and energy source. 


\section{SPECIFIC AIMS}

AnkB is the only ankyrin effector to be translocated from the site of extracellular bacterial attachment [129] and it has been shown that polyubiquitinated proteins appear within 15 minutes of bacterial attachment. Therefore, it is likely that AnkB is farnesylated immediately following bacterial attachment, and interacts with the SCF1 E3 ubiquitin ligase complex to cause polyubiquitination of its substrates. The proteasomal degradation of the polyubiquitinated substrates will generate an increase in free cellular amino acids to inhibit differentiation into the non-replicative phase and power intracellular replication.

L. pneumophila is auxotrophic for seven amino acids, therefore acquisition of host cell amino acids is critical for intracellular survival and replication. Further characterization of the ubiquitinated proteins localized to the LCV will provide potential substrates of AnkB that can be further investigated to determine their role during L. pneumophila infection.

I hypothesize, that once L. pneumophila makes contact with the host cell, AnkB is rapidly translocated and farnesylated which allows it to integrate into the LCV membrane and interact with the host SCF1 E3 ubiquitin ligase complex to cause polyubiquitination of the LCV. 
To test this hypothesis my specific aims are:

Specific Aim 1: Study the kinetics of AnkB translocation upon attachment of extracellular L. pneumophila, and determine if the host cell farnesylation and ubiquitination machineries are localized and manipulated at the site of extracellular bacterial attachment.

Specific Aim 2: Characterize the complete and ubiquitinated proteome of the Legionellacontaining vacuole from human macrophages.

Specific Aim 3: Determine if AnkB is ubiquitinated and the type of polyubiquitin chain linkage. 


\section{MATERIALS AND METHODS}

\section{Bacterial strains, cell cultures}

L. pneumophila strain AA100/130b (ATCC BAA-74), the isogenic ankB and dotA mutants were grown on buffered charcoal yeast extract (BCYE) agar plates for 3 days at $37^{\circ} \mathrm{C}$ prior to use in infections as described previously [131]. Escherichia coli strain DH5 $\alpha$ was used for cloning and plasmid preparation purposes. Human monocyte-derived macrophages (hMDMs) and U937 cells were cultured using RPMI1640 media as we described previously [238]. HEK293T cells were grown in Dulbecco's modified Eagle's medium (DMEM) supplemented with 10\% FBS (BioWest) and 200mm L-glutamine (Corning) at $37^{\circ} \mathrm{C}$ in $5 \% \mathrm{CO}_{2}$ atmosphere.

\section{Plasmids and DNA manipulations.}

The $a n k B$ gene was cloned into the mammalian expression vector p3XFlag-CMV-10. Generation of the $3 \mathrm{X}$-Flag $a n k B-{ }^{9} L^{10} P, 3 \mathrm{X}$-Flag ankB$\triangle F b o x$ and $3 \mathrm{X}$-Flag-tagged AnkH were described previously [176, 238]. The HA-tagged-Trim21 was a kind gift from Dr. Yong-Jun Liu at the University of Baylor. The plasmid PXDC61M, which contains the blaM gene encoding the mature form of TEM-1 beta-lactamase, was obtained from Dr. Zhao-Qing Luo at Purdue University. The ankB gene was PCR amplified with restriction enzymes and cloned in frame with the beta-lactamase at the BamHI-XbaI sites to generate a transcriptional fusion protein. The resulting plasmid was introduced into L. pneumophila strain AA100/130b and the 
dotA mutant. To verify expression of the fusion proteins in L. pneumophila, strains harboring pXDC61M were grown on BCYE containing chloramphenicol, IPTG $(0.5 \mathrm{mM})$ and analyzed by western blotting. Protein from $1 \mathrm{X} 10^{8}$ bacteria was transferred to nitrocellulose membranes and detected by western blot with a primary monoclonal antibody specific to TEM-1 $\beta$ lactamase (QED Bioscience) and anti-mouse peroxide conjugate as a secondary antibody.

\section{Quantitative Real Time PCR}

Real-time qPCR on attached bacteria was performed as described previously [131, 177]. Briefly, hMDMs were plated at a density of $5 \times 10^{5}$ in 24 well plates and treated with 1 $\mu \mathrm{M}$ cytochalasin $\mathrm{D}$ for 30 minutes prior to infection. The hMDMs were then infected with WT bacteria at a multiplicity of infection (MOI) of 10 for $0,7.5$ or 15 minutes, and intimate and synchronized attachment was achieved by centrifugation at 1000rpm for 5 minutes. To assess RNA expression levels of ankB, mompS and I6S RNA in response to attachment to hMDMS total RNA was extracted from infected cells at the indicated time points using the RNeasy Mini Kit (Qiagen, Valencia, CA) as recommended by the manufacturer. Total RNA was treated with DNase I (Ambion, Austin, TX) at $37^{\circ} \mathrm{C}$ for $30 \mathrm{~min}$. Equal amounts of total RNA from infected cells were used for cDNA synthesis using Superscript III Plus RNase H reverse transcriptase (RT) (Invitrogen, CA) and random primers. Real-time qPCR was done in triplicate using the Power SYBR Green PCR Master Mix Kit in a $20 \mu$ reaction volume, as recommended by the manufacturer (Applied Biosystems, CA), using specific primers. The PCR conditions were 2 min at $94^{\circ} \mathrm{C}$ initially followed by, $10 \mathrm{sec}$ at $96^{\circ} \mathrm{C}$ and $20 \mathrm{~s}$ at $47^{\circ} \mathrm{C}$ and $15 \mathrm{~s}$ at $72^{\circ} \mathrm{C}$ for 40 cycles. Changes in mRNA expression were determined by the comparative CT method (threshold cycle number at the cross-point between amplification plot and threshold) and 
values were normalized to $16 \mathrm{~S}$ RNA. Negative or positive values were considered as downregulation or up-regulation when there was a minimum of two-fold difference of gene expression.

\section{Preferential plasma membrane permeabilization and loading of the cytosol with antibodies}

Human monocyte-derived macrophages (hMDMs) were isolated and maintained as described previously [131]. Monocytes were seeded in 24 well plates at 1 X10 6 cells/well. Cells were treated with cytochalasin $\mathrm{D}(5 \mu \mathrm{g} / \mathrm{ml})$, an actin polymerization inhibitor, prior to infection and throughout the experiment. hMDM plasma membranes were selectively permeabilized for 5 minutes at room temperature with a RPMI 1640 solution containing digitonin $(50 \mu \mathrm{g} / \mathrm{ml})$ as well as anti-AnkB antiserum [135]. Following plasma membrane permeabilization cells were extensively washed with media and infected with wild type L. pneumophila as well as the $a n k B$, $a n k B{ }^{169} C / A$ and $\operatorname{dot} A$ mutant strains at a MOI 10 for 15 minutes. Monocytes were extensively washed with media and incubated an additional 30 minutes for antibody-antigen interaction. Cells were then fixed with $3.7 \%$ formaldehyde for 15 minutes at room temperature. To ensure cytochalasin D inhibited phagocytosis and bacteria remained extracellular, antibody labeling of L. pneumophila with specific rabbit polyclonal anti-serum was performed prior to permeabilization, followed by Alexa Fluor 488 donkey anti-mouse secondary antibody. (Invitrogen). Cells were then permeabilized with $0.1 \%$ Triton $\mathrm{X} 100$ for 10 minutes at room temperature, followed by anti-AnkB antiserum detection by Alexa Fluor 555-conjugated donkey anti-rabbit IgG (Invitrogen). 


\section{TEM translocation assay}

The U937 cells were grown in RPMI 1640 containing 10\% FBS and seeded in black clear- bottom 96 well plates at $1 \times 10^{5}$ cells/well and treated with phorbol ester (PMA) at $48 \mathrm{~h}$ prior to infection. L. pneumophila strains containing the TEM-1 fusion proteins were grown for 3 days on BCYE containing chloramphenicol $(5 \mu \mathrm{g} / \mathrm{ml})$ and then streaked on BCYE containing chloramphenicol and $0.5 \mathrm{mM}$ Isoprpyl $\beta$-D-1-thiogalactopyranoside (IPTG) to induce expression of the fusion proteins. Cell monolayers were loaded with the $\beta$-lactamase substrate CCF4 by adding 20 $\mu 1$ of 6X CCF4-AM solution (LiveBLAzer ${ }^{\text {TM }}$-FRET B/G Loading Kit, Invitrogen) containing $0.1 \mathrm{M}$ probenecid. Cells were incubated with the solution for 2 hours at room temperature. U937 cells were treated with the actin polymerization inhibitor, cytochalasin $\mathrm{D}(5 \mu \mathrm{g} / \mathrm{mL})$, for $1 \mathrm{hr}$ prior to infection and maintained throughout the infection using different MOIs. Plates were centrifuged (300g, $5 \mathrm{~min})$ to initiate bacterial-cell contact and incubated at $37^{\circ} \mathrm{C}$ for 10 minutes. Fluorescence was quantified on a BioTek Synergy HT Microplate Reader with excitation at 405nm, and emission was detected at 460nm and 530nm. Bacterial effector translocation was determined by the emission ratio $460 \mathrm{~nm} / 530 \mathrm{~nm}$ to normalize the $\beta$-lactamase activity to non-infected substrate loaded cells.

\section{Recruitment of host farnesylation and ubiquitination machinery to sites of $L$. pneumophila attachment.}

A total of $5 \times 10^{5} \mathrm{hMDMs}$ on glass coverslips in 24 well plates were pretreated for 30 minutes with cytochalasin D $(5 \mu \mathrm{g} / \mathrm{ml})$ and then infected with wild type L. pneumophila and the $a n k B$ and $\operatorname{dot} A$ mutants at an MOI of 10 for 15 minutes. Processing of infected cells for confocal microscopy was performed. Briefly, fixed and permeabilized cells were blocked for 
1h with $3 \%$ BSA-PBS and then mouse anti-L. pneumophila anti-serum (1/1000 dilution), and anti-Skp1, anti-Cul1, anti-FT $\alpha$, anti-RCE1 and anti-IcmT antibodies (1/200 dilution) (Abcam, Cambridge, MA) were added to $3 \%$ BSA-PBS and incubated at room temperature for $1 \mathrm{~h}$. Following extensive washing with 3\% BSA-PBS, bound antibodies were detected with Alexa Fluor 488 or 555-conjugated donkey anti-rabbit or mouse IgG antibodies (Invitrogen, Carlsbad, CA) for 1h. Following this, the glass cover slips were mounted on glass slides using ProLong Gold anti-fade reagent (Invitrogen, Carlsbad, CA). The fixed cells were examined with an Olympus FV1000 laser scanning confocal microscope as described previously [238]. On average, 8-15 0.2 um serial Z sections of each image were captured and stored for further analyses, such as cropping and centering the images, using Adobe Photoshop CS5.

\section{Gas Chromatography-Mass Spectrometry analyses of free amino acids}

The cellular levels of free amino acids was determined as part of the global metabolomics profile. The hMDMs cells were seeded in 6 well plates at $1 \times 10^{6}$ and prior to infection the cells were treated with $1 \mu \mathrm{M}$ cytochalisin $\mathrm{D}$ for 30 minutes. The hMDMs were infected with WT or ankB mutant L. pneumophila at an MOI of 100 for $1 \mathrm{~h}$ and the infected cells were lysed in aqueous $90 \%$ methanol. Lysates were stored at $-20^{\circ} \mathrm{C}$ for $1 \mathrm{~h}$ and then centrifuged $\left(21000 \mathrm{x}\right.$ g at $\left.4^{\circ} \mathrm{C}\right)$ for $10 \mathrm{~min}$. The resulting supernatants were dried using a SpeedVac and prepared for GC-MS.

All GC-MS analysis was performed at the University of Utah Metabolomics core facility using a Waters GCT Premier mass spectrometer fitted with an Agilent 6890 gas chromatograph and a Gerstel MPS2 autosampler. The dried samples were suspended in $40 \mu \mathrm{L}$ of a $40 \mathrm{mg} / \mathrm{mL}$ O-methoxylamine hydrochloride in pyridine and incubated for one hour at 
$30^{\circ} \mathrm{C}$. A $25 \mu \mathrm{L}$ sample of this solution was transferred to auto-sampler vials followed by the addition of $N$-methyl- $N$-trimethylsilyltrifluoracetamide and further incubated for 30 minutes at $37^{\circ} \mathrm{C}$ with shaking. A $1 \mu \mathrm{L}$ sample was injected to the gas chromatograph inlet in the split mode set to a 10:1 ratio. Injector temperature was held at $250^{\circ} \mathrm{C}$. The gas chromatograph had an initial temperature of $95^{\circ} \mathrm{C}$ for one minute followed by a $40^{\circ} \mathrm{C} / \mathrm{min}$ ramp to $110^{\circ} \mathrm{C}$ with a hold time of 2 minutes. This was followed by a second $5^{\circ} \mathrm{C} / \mathrm{min}$ ramp to $250^{\circ} \mathrm{C}$ then a third ramp to $350^{\circ} \mathrm{C}$ and a final hold time of 3 minutes. A 30 m Restek Rxi-5 MS column with a 5 m long guard column was employed for analysis. Data were collected by MassLynx 4.1. Data analysis for free cellular amino acids was performed using QuanLynx which quantified the area under the curve for each amino acid. All data was saved to an Excel spread sheet for further analysis. This analysis includes most of the amino acids, but His is difficult to detect and Cys makes disulfide bonds with proteins and is also difficult to detect. In our samples, Arg and Asn were not detectable by the methodology used. The GC-MS analysis gives relative results of the area under the curve for the same amino acid, and is not quantitative relative to other amino acids within the same sample. Since the GC-MS analyses compare levels of the same metabolite/amino acid between different samples/treatments, the results are presented as a ratio of infected/un-infected cells.

\section{..Legionella-containing vacuole purification}

A total of $6 \times 10^{8} \mathrm{U} 937$ macrophages were plated in T175cm flask in $60 \mathrm{ml}$ RPMI media supplemented with $10 \%$ FBS. At a multiplicity of infection of 50 bacteria per cell Legionella containing vacuoles were formed by internalization of bacteria diluted in $20 \mathrm{ml}$ RPMI media. Internalization of the bacteria was performed for $30 \mathrm{~min}$ at $37^{\circ} \mathrm{C}$ 
under 5\% CO2. Cells were washed $3 \mathrm{X}$ in PBS and then incubated in growth media for 4 hrs at $37^{\circ} \mathrm{C}$ under $5 \% \mathrm{CO} 2$. After 4 hrs cells were washed $3 \mathrm{X}$ in $\mathrm{PBS}\left(4^{\circ} \mathrm{C}\right)$ and scraped into a $50 \mathrm{ml}$ screw cap centrifuge tube and pelleted at $4^{\circ} \mathrm{C}$ for $5 \mathrm{~min}$ at $450 \mathrm{~g}$. Cells were resuspended in homogenization buffer (250mM sucrose, 20mM Hepes/KOH (pH7.2) $+0.5 \mathrm{mM}$ EGTA $(\mathrm{pH} 8.0)$ ) and pelleted by centrifugation at $675 \mathrm{~g}$ for 6 minutes at $4^{\circ} \mathrm{C}$. Cells were then resuspended in homogenization buffer with protease inhibitors (Roche cocktail) at $2 \times 10^{\wedge} 8 / \mathrm{ml}$. Cells were lysed with a dounce homogenizer on ice and visualized under light and confocal microscopy to ensure effective cell lysing and Legionella vacuole integrity. Whole cells and nuclei were then pelleted in an $1.5 \mathrm{ml}$ tube for $3.5 \mathrm{~min}$ at $344 \mathrm{~g}$. The supernatant was placed in a new $1.5 \mathrm{ml}$ tube and centrifuged for $3.5 \mathrm{~min}$ at $344 \mathrm{~g}$ resulting in the post nuclear supernatant (PNS). The PNS was brought to a final concentration of $39 \%$ sucrose. The sucrose solutions for the step gradient were made in w/v in $20 \mathrm{mM}$ Hepes $/ \mathrm{KOH}$ ( $\mathrm{pH} 7.2)$. The sucrose step gradient was made by layering the PNS (39\% sucrose) onto $2 \mathrm{ml} 55 \%$ sucrose layered onto $1 \mathrm{ml} 65 \%$ sucrose in a $14 \mathrm{~mm}$ X $89 \mathrm{~mm}$ Beckman ultracentrifuge tube. We then layered $2 \mathrm{ml} 10 \%$ sucrose onto $2 \mathrm{ml} 25 \%$ sucrose solution onto the PNS. The sucrose gradient was centrifuged for $1 \mathrm{hr}$ at $100,000 \mathrm{~g}$ at $4^{\circ} \mathrm{C}$ in a swinging bucket rotor (Beckman SW41). The LCVs were isolated from the 55\%-65\% interface using a $16 \mathrm{~g}$ needle and not disturbing any other fraction. LCVs were placed into $10 \mathrm{ml} \mathrm{PBS}\left(4^{\circ} \mathrm{C}\right)$ and centrifuged at $40,000 \mathrm{~g}(\mathrm{SW} 41)$ for $30 \mathrm{~min}$ at $4^{\circ} \mathrm{C}$. Pelleted LCVs were solubilized in $1 \%$ Triton X100 in PBS for 30 minutes on ice. Following centrifugation at 10,000g for 5 minutes to pellet bacteria, the supernatant containing eukaryotic proteins associated with the LCV was stored at $-80^{\circ} \mathrm{C}$. 


\section{...Antibodies and Confocal Microscopy for LCV isolation}

Isolated LCVs were plated onto 24 well coverslips pretreated with poly-L-lysine and allowed to adhere for $1 \mathrm{hr}$. Extravacuolar L. pneumophila were labeled with a rabbit anti- L. pneumophila antibody prior to permeabilization for $1 \mathrm{hr}$. LCVs were then permeabilized with methanol $\left(-20^{\circ} \mathrm{C}\right)$ for 5 minutes, blocked with $3 \%$ BSA for $1 \mathrm{hr}$ and then labeled with a mouse anti-L. pneumophila antibody for $1 \mathrm{hr}$. Secondary antibodies used were Alexa Flour goat anti-mouse 488 and Alexa Flour goat anti-rabbit 555. Polyubiquitinated proteins present on the LCVs were labeled with a mouse antipolyubiquitin antibody (Enzo Life Sciences) for $1 \mathrm{hr}$ prior to vacuole membrane permeabilization and visualized with Alexa Flour goat anti-mouse 488 .

\section{Protein Digestion for Mass Spectrometry}

For Mass Spectrometry analysis, TCA precipitated proteins were resuspended in $8 \mathrm{M}$ urea, 50mM Tris $\mathrm{pH}$ 8.5. Resuspended peptides were diluted 1:1 with 50mM Tris $\mathrm{pH} 8.5$ to lower the urea concentration to $4 \mathrm{M}$ prior to digestion with endoproteinase Lys-C (10ng/uL) for 8hrs. Digestions were further diluted 4:1 with 50mM Tris $\mathrm{pH} 8.5$ to lower the urea concentration to $1 \mathrm{M}$ urea prior to digestion with trypsin $(5 \mathrm{ng} / \mathrm{uL})$ for 8hr. Peptides were desalted using a 50mg tC18 Sep-Pak (Waters). 


\section{Ubiquitinated Protein immunoprecipitation for ubiquitinated LCV proteome}

analysis

Ubiquitinated peptides were immunoprecipitated using ubiquitin motif immunoaffinity beads (Cell Signaling Technology) as recommended by the manufacturer with the following modifications [242]. Desalted peptides were dissolved in 1.0-1.4 mL of IAP buffer (50 mM MOPS-NaOH, pH 7.2, $10 \mathrm{mM} \mathrm{Na2HPO4,} 50 \mathrm{mM}$ $\mathrm{NaCl}$ ). The $\mathrm{pH}$ was adjusted to 7.2 with $1 \mathrm{M} \mathrm{NaOH}$. The peptide solution was cleared by centrifugation for $5-10 \mathrm{~min}$ at $13,200 \mathrm{~g}$. The supernatant was transferred to a new tube and cooled on ice for 10 minutes. The cooled peptide solution was transferred to the tube with the antibody beads and incubated on a rotator at $4^{\circ} \mathrm{C}$ for $60 \mathrm{~min}$. The beads were washed 4 times with $1 \mathrm{~mL}$ IAP buffer, followed by 1 wash with $1 \mathrm{~mL}$ water. The peptides were eluted from the beads by adding $55 \mu 15 \%$ Formic Acid (FA), mixed and incubated at room temperature for $10 \mathrm{~min}$. The beads and elution buffer were transferred to a Teflon spin column and the eluate was collected by centrifugation. The beads were washed with $45 \mu 10.1 \%$ TFA and combined with the first eluate [242]. The peptides were desalted using StageTips [243] and nanoLC-MS2 data was collected.

\section{nanoLC-MS2 data collection}

In collaboration with Dr. Steve Gygi, Dr. Ryan Kunz and Ross Tomaino at the Harvard University Taplin Mass Spectrometry Facility mass spectrometry data were collected on an Orbitrap Fusion mass spectrometer equipped with an easy nano-LC 1000 for sample handling and liquid chromatography. Peptides were separated on a 75 $\mu \mathrm{m} \times 30 \mathrm{~cm}$ hand-pulled fused silica microcapillary column with a needle tip diameter 
less than $10 \mu \mathrm{m}$ and packed with $1.8 \mu \mathrm{m} 120 \AA$ GP-C18 beads from Sepax Technologies Inc. The column was equilibrated with buffer A ( $3 \% \mathrm{ACN}+0.125 \% \mathrm{FA})$. Peptides were loaded onto the column at $100 \%$ buffer A. Separation and elution from the column was achieved using a 90 min 3-25\% gradient of buffer B (100\% ACN + 0.125\% FA). Survey scans of peptide precursors from 400 to $1400 \mathrm{~m} / \mathrm{z}$ were performed at $120 \mathrm{~K}$ resolution (at $200 \mathrm{~m} / \mathrm{z}$ ); AGC, 50k; max injection time, 100ms; monoisotopic precursor selection turned on; charge state, 2-6; dynamic exclusion, $45 \mathrm{~s}$ with a $10 \mathrm{ppm}$ tolerance. Tandem MS was performed in top speed mode (2 second cycles) starting with the most intense precursor having an intensity greater than $5 \mathrm{k}$. Parent ions were isolated in the quadrupole $(0.7 \mathrm{~m} / \mathrm{z}$ isolation window). Collision induced dissociation was performed in the ion trap with a rapid scan rate; $35 \%$ collision energy; AGC, 10k; max injection time, 35ms; parallelizable time was turned on.

\section{Mass spectrometry data analysis}

A suite of in-house software tools, created by Dr. Steve Gygi, was used for .RAW file processing, controlling peptide and protein false discovery rates, and assembling peptide level data into protein level data [242, 244]. The MS/MS spectra were searched using the SEQUEST algorithm [245] against a composite protein database consisting of all protein sequences from the Uniprot human database (88,501 proteins) along with common contaminating proteins (111 proteins) in both the forward and reverse direction. Sequest parameters used to search the MS data were: precursor tolerance, 50ppm; fragment ion tolerance, 1Da; fully tryptic; 2 missed cleavages; variable modifications of oxidized methionine (15.9949 Da), alkylation of cysteine (57.0214), 
and diGlycine motif on lysine (114.0429). A target-decoy strategy was used to determine false discovery rates [246]. The peptide-level false discovery rate was restricted to $<1 \%$ by using linear discriminate analysis based on several different SEQUEST parameters including Xcorr $\geq 1.0$, deltaXcorr, charge state, and a minimum peptide length of 7 amino acids [244]. An algorithm similar to Ascore was used for diGlycine localization and site quantification [242, 247]. The localization score is based on the Ascore algorithm, where a localization score $\geq 19$ indicated $>99 \%$ certainty in site localization [247]. Protein identifications for the complete proteome of the WT strain and $a n k B$ mutant strain are based on at least 2 unique peptides.

\section{MetaCore Software Analysis}

MetaCore is an integrated software suite for functional analysis of various aspects of screening data [248, 249]. MetaCore software determines the most significant relationships among the proteins analyzed such as Pathway Maps, GO Processes, Process Networks and Metabolomic Networks. For our analysis the software determined the most significant relationships shared among the WT strain LCV proteome and a separate analysis for the $a n k B$ mutant strain. MetaCore software is available online and was obtained through the University of Louisville license. The excel spreadsheet containing the complete WT strain LCV proteome and the ankB mutant stain LCV proteome were separately uploaded into the start page and an enrichment analysis workflow was generated. 


\section{Transfection of HEK293T cells.}

The ankB gene was cloned into the mammalian expression vector p3XFlag-CMV-10.

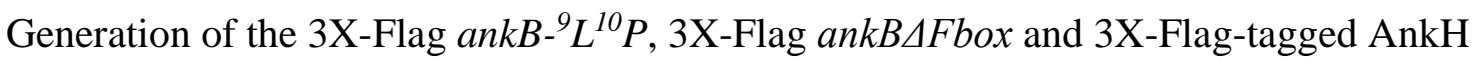
were described previously [176, 238]. The HA-tagged-Trim21 was a kind gift from Dr. Yong-Jun Liu at the University of Baylor. HEK293T cells were grown to 70\% confluency and plated onto poly-L lysine treated 6 well plates. Following $24 \mathrm{~h}$ incubation HEK293T cell monolayers were transfected with 2ug plasmid DNA/well using polyethylenimine (Polysciences) for $24 \mathrm{~h}$.

\section{Cycloheximide inhibition}

HEK293T cells plated in 6 well plates were transfected with 3XFlag-AnkB for 24 hr. The proteasomal inhibitor MG132 (Selleckchem) used at 20uM was added to the indicated cells 2 hours prior to cycloheximide treatment at $100 \mathrm{ug} / \mathrm{ml}$. At indicated time points cells were lysed and subjected to SDS-PAGE and immunoblotting with anti-Flag and anti-actin antibodies.

\section{In vivo co-immunoprecipitation}

HEK293T cells were transfected with3X-Flag AnkB, AnkB ${ }^{9} \mathrm{~L}^{10} \mathrm{P}, \mathrm{AnkB} \Delta \mathrm{Fbox}$, AnkH, HA-tagged Trim 21 for $24 \mathrm{hr}$ and collected in lysis buffer containing $50 \mathrm{mM}$ Tris pH 7.4, $0.25 \mathrm{M} \mathrm{NaCl}, 0.5 \%$ Triton X-100, $1 \mathrm{mM}$ EDTA, 50mM NaF, $0.1 \mathrm{mM} \mathrm{Na}_{3} \mathrm{VO}_{4}$ and EDTA-free protease inhibitor cocktail (Roche). Flag-tagged and HA-tagged proteins were immunoprecipitated using anti-Flag M2 agarose (Sigma) or anti-HA affinity gel (Sigma) according to the manufacturer's instructions. 


\section{Antibodies and Western Blot}

Immunoprecipitated proteins were heated at $99^{\circ} \mathrm{C}$ for 5 minutes in sample buffer (Pierce) and separated by 10.4-15\% SDS-PAGE electrophoresis and transferred to a PVDF membrane. Anti-Flag (Sigma) used at 1:1000, anti-actin (Proteintech) used at 1:15000, anti-ubiquitin (Cell Signaling) used at 1:1000, anti-K $\mathrm{K}^{48}$ ubiquitin (Cell Signaling) used at 1:1000, anti- $\mathrm{K}^{63}$ ubiquitin (Cell Signaling) used at 1:1000, anti-M45 used at 1:50 were incubated overnight in $5 \%$ milk at $4^{\circ} \mathrm{C}$. Anti-HA (Cell Signaling) was used at 1:1000 incubated in 5\% BSA overnight at $4^{\circ} \mathrm{C}$. 


\title{
RESULTS
}

\author{
Section 1. Rapid Nutritional Remodeling of Macrophages \\ Upon Attachment of Legionella pneumophila
}

\section{Triggering transcription of ankB upon attachment of $L$. pneumophila to human macrophages}

Upon entry of the ankB mutant to macrophages or amoeba, it exhibits a dramatic starvation response and differentiation into the non-replicative phase, but both phenotypes are circumvented by amino acids supplementation [86]. Similar phenotypes are also exhibited by the WT strain upon entry into proteasome-inhibited cells, and in both cases the respective phenotypes are circumvented upon supplementation of amino acids [86]. L. pneumophila is auxotrophic for seven amino acids, therefore, acquisition of host cell amino acids is critical to provide the carbon and energy needed during intracellular replication [250]. Previous data indicates AnkB as the major contributor to host amino acid retrieval, therefore, translocating AnkB upon intimate attachment with the host cell will increase the host cell amino acid levels prior to LCV formation [86]. Investigating the function of AnkB from attached extracellular bacteria can lead clues into how L. pneumophila manipulates the host cell polyubiquitination machinery to increase the host amino acid levels prior to bacterial entry.

We tested the hypothesis that WT L. pneumophila likely employs AnkB during initial stages of interaction with the host cell to circumvent the amino acids starvation response and the associated phenotypic modulations. We determined whether attachment of L. pneumophila 
to host cells triggered transcription of AnkB. To determine this, human monocytes-derived macrophages (hMDMs) were pre-treated with cytochalasin $\mathrm{D}$ for 30 minutes to block phagocytosis and then infected with WT L. pneumophila at an MOI of 10 for $0,7.5$ and 15 minutes. This bacterial attachment protocol resulted in attachment of 1-2 bacteria/ cell in 50\% of the cells in the monolayers. Block of bacterial entry into hMDMs by cytochalasin D was confirmed by treatment of the infected cytochalasin D-treated monolayers by gentamicin, indicating that the bacteria were extracellular [86]. Following RNA purification and cDNA synthesis, expression of the $a n k B$ gene was determined by Real-Time qPCR using expression of the 16S RNA as an internal control, as we described previously [131, 176, 251]. Expression of the constitutively expressed mompS gene was used as a control. Compared to expression of ankB at 0 minutes post-attachment, its expression was increased by 9 -fold and 26 -fold at 7.5 and 15 minutes, respectively, post-bacterial attachment (Fig. 1). During the course of the attachment experiment no change was observed in expression of mompS (Fig. 1). These data show that transcription of $a n k B$ is triggered immediately upon attachment of L. pneumophila to hMDMs. 


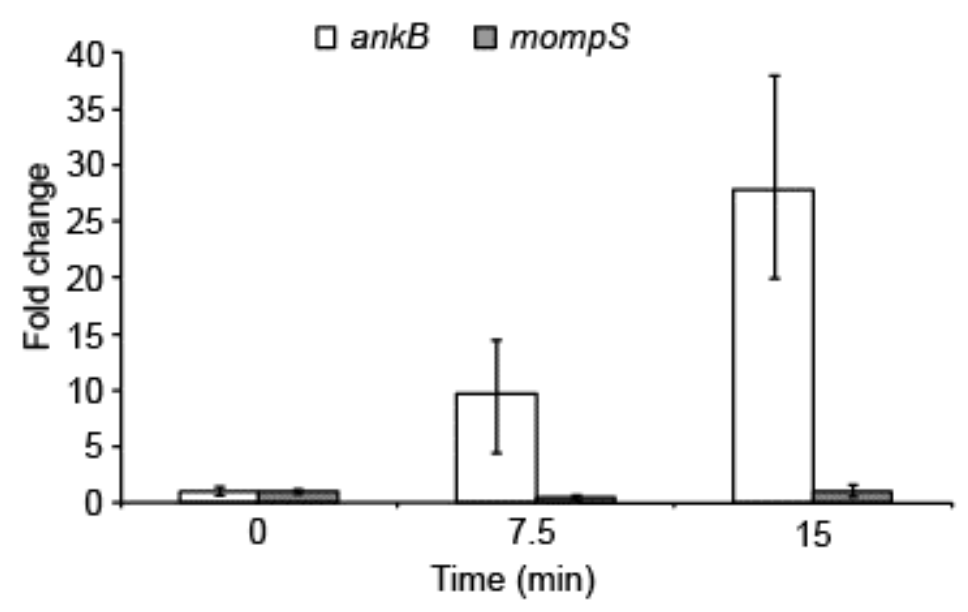

Figure 1: Expression of ankB upon intimate attachment to hMDMs. Quantitative RealTime PCR of ankB gene transcription by L. pneumophila at $0,7.5$ and 15 minutes postattachment to hMDMs. The hMDMs were pre-treated with cytochalasin D and infected for the indicated time periods followed by total isolation of RNA and RT-PCR. Transcription of ankB, or the control gene mompS was normalized to $16 \mathrm{~S}$ RNA levels. Fold changes in gene expression were compared to levels measured at time 0 minutes. Error bars indicate SEM. The data are representative of three independent experiments.

\section{Translocation of AnkB into macrophages upon bacterial attachment}

To determine temporal and spatial translocation of AnkB upon intimate attachment of $L$. pneumophila to macrophages, we generated a $L$. pneumophila strain expressing a $\beta$ lactamase-AnkB reporter fusion construct to monitor real-time translocation in cells pre-loaded with the CCF4 fluorometric $\beta$-lactamase substrate [132]. The human macrophage U937 cell line preloaded with CCF4 was pretreated with cytochalasin D, and then infected with $L$. pneumophila expressing $\beta$-lactamase fusions at various MOIs for 15 minutes (Fig. 2). 
Hydrolysis of CCF4 was measured by quantifying blue (460nm) and green (530nm) fluorescence emission, and is reported as the $460 \mathrm{~nm} / 530 \mathrm{~nm}$ ratio where a ratio greater than 1 represents positive translocation. The data showed that AnkB was equally translocated into untreated or cytochalasin D-treated U937 macrophages. Translocation of AnkB by attached extracellular bacteria was completely dependent on a functional Dot/Icm T4SS, since the dotA translocation-defective mutant failed to translocate the effector (Fig. 2). As expected, increasing the MOI resulted in increased AnkB translocation in a dose-response manner, as evident by greater CCF4 hydrolysis (Fig. 2). No translocation was detected for the fatty acid biosynthetic enzyme, enoyl-CoA reductase (FabI), which was used as a negative control (Fig. 2) [132]. These data show that attachment of L. pneumophila to macrophages triggers rapid translocation of the AnkB effector into the host cell.

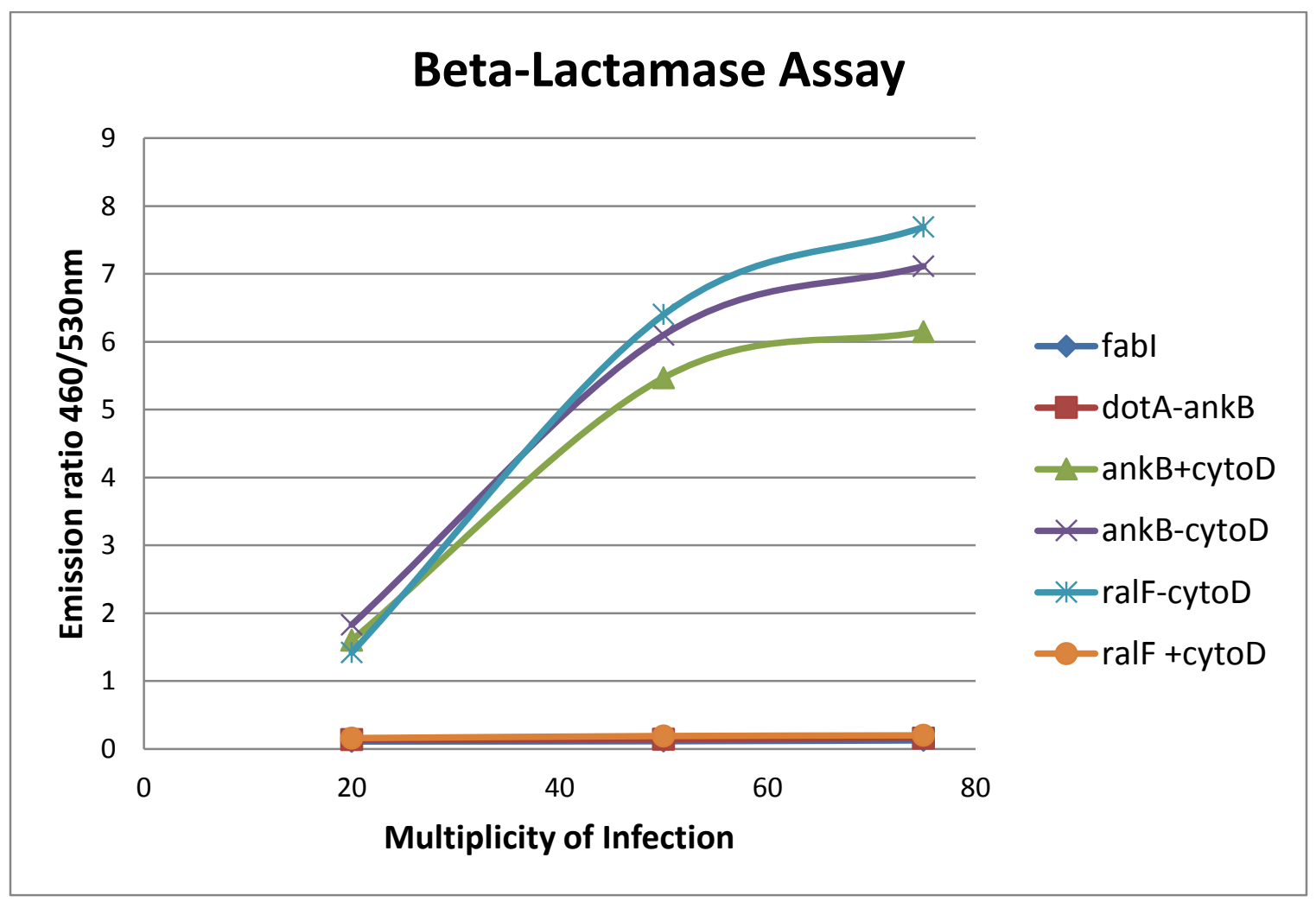


Figure 2: Translocation of $\beta$ lactamase -AnkB fusion protein by L. pneumophila upon intimate attachment to U937 cells. Cytochalasin D-treated U937 cells were infected with increasing MOIs of L. pneumophila strains expressing various $\beta$ lactamase-fusion constructs and translocation was measured by monitoring hydrolysis of the fluorogenic substrate CCF4. A $460 \mathrm{~nm} / 530 \mathrm{~nm}$ ratio greater than 1 indicates translocation of the fusion construct. Data represent mean values of three independent experiments.

\section{Host-mediated farnesylation and anchoring of AnkB to the cytosolic side of the plasma membrane beneath attachment sites of extracellular bacteria}

We decided to determine the cellular location of the native AnkB effector injected by attached extracellular bacteria. Using anti-AnkB antiserum on cytochalasin D-treated infected cells to detect cellular location of AnkB injected by attached extracellular bacteria was difficult to interpret by confocal microscopy. This was due to our findings that the injected AnkB was not detected in the cytosol but seemed to be localized beneath the attached extracellular WT bacteria, which also bound the anti-AnkB antibody. The resolution was not sufficient to differentiate injected AnkB localized intracellularly beneath bacterial attachment from AnkB contained in the attached extracellular bacteria. Since the $\beta$-lactamase-AnkB reporter fusion was clearly injected by attached extracellular bacteria (Fig. 2), our microscopy findings suggested that the injected AnkB was likely localized exclusively beneath bacterial attachment sites. 
To overcome the above mentioned caveat and to determine whether the injected native AnkB by attached extracellular bacteria was located beneath bacterial attachment sites, the cytosol of live hMDMs was pre-loaded with anti-AnkB antibodies prior to bacterial attachment. This would allow the antibody to bind AnkB upon its injection by attached extracellular bacteria [252-254]. This strategy also provides a clear and more solid interpretation of the data, since the anti-AnkB antibody is loaded to the host cell cytosol prior to inoculation of the bacteria. To load the host cell cytosol with the anti-AnkB antibodies prior to infection, the plasma membrane of live hMDMs was preferentially permeabilized with a low concentration of digitonin [252-254]. After loading the cells with the antibody and allowing the cells to heal the membrane damage for a few minutes, integrity of the plasma membrane was confirmed by impermeability to Trypan blue (data not shown). Without digitonin treatment, the plasma membrane of hMDMs was impermeable to anti-AnkB antibodies, as expected (Fig. 3). The antibody-loaded hMDMs were treated with cytochalasin D to prevent phagocytosis and then infected at a MOI of 10 with WT L. pneumophila or the isogenic mutants, dotA or ankB. The cells were then fixed and processed for confocal microscopy. This bacterial attachment protocol resulted in an average attachment of 1-2 bacteria/ cell in $\sim 50 \%$ of the cells in the monolayers. To allow differentiation between extracellular and intracellular bacteria, extracellular L. pneumophila were labeled with specific antibodies prior to permeabilization of the infected cells. When the hMDMs were permeabilized with digitonin and loaded with anti-AnkB antibodies, the loaded antibody was detectable as red patches throughout the cytosol of $\sim 98 \%$ of the cells, indicating successful loading of the cells with anti-AnkB antibodies to prior to infection (Fig. 3). When hMDMs were infected with wild type L. pneumophila, $52 \%$ of attached extracellular bacteria co- 
localized with AnkB exclusively beneath the site of bacterial attachment (Fig. 3). The $48 \%$ of extracellular bacteria that do not not co-localize with anti-AnkB could be due to the low amount of AnkB translocated, the level of antibody detection, or the bacteria not yet translocating AnkB into the cell. In addition, AnkB must be farnesylated to anchor into the plasma membrane beneath attached extracellular bacteria, therefore, multiple biological and technical reasons can account for the $48 \%$ of extracellular bacteria that lack co-localization to the site of extracellular bacterial attachment. As expected, the dotA translocation-defective mutant and the ankB mutant did not co-localize with AnkB (5\% and 0\% respectively) (Student $t$-test, $P<0.007$ and 0.003 , respectively) (Fig. 3).

Since AnkB is hydrophilic and its anchoring to the LCV membrane is mediated by host farnesylation within amoeba and macrophages [135, 255], we determined whether host farnesylation was required for the exclusive localization of AnkB to the cytosolic side of the plasma membrane beneath the sites of bacterial attachment. The cytosol of hMDMs was preloaded with anti-AnkB antiserum prior to infection, as described above. Cytochalasin-D treated cells were infected by the farnesylation-defection $a n k B-{ }^{169} \mathrm{C}-\mathrm{A}$ substitution mutant in the CaaX motif, which has been shown to be translocated into the host cell [135]. The data showed that infection by the $a n k B-{ }^{169} \mathrm{C}-\mathrm{A}$ substitution mutant resulted in failure to anchor AnkB to the plasma membrane beneath bacterial attachment sites (Student $t$-test, $P<0.005$ ) (Fig. 3). Therefore, host farnesylation anchors the injected AnkB by attached extracellular $L$. pneumophila to the cytosolic side of the plasma membrane directly and exclusively beneath bacterial attachment sites. This is the first demonstration of farnesylation-mediated anchoring of an injected bacterial effector to the inner leaflet of the plasma membrane beneath bacterial attachment sites. 

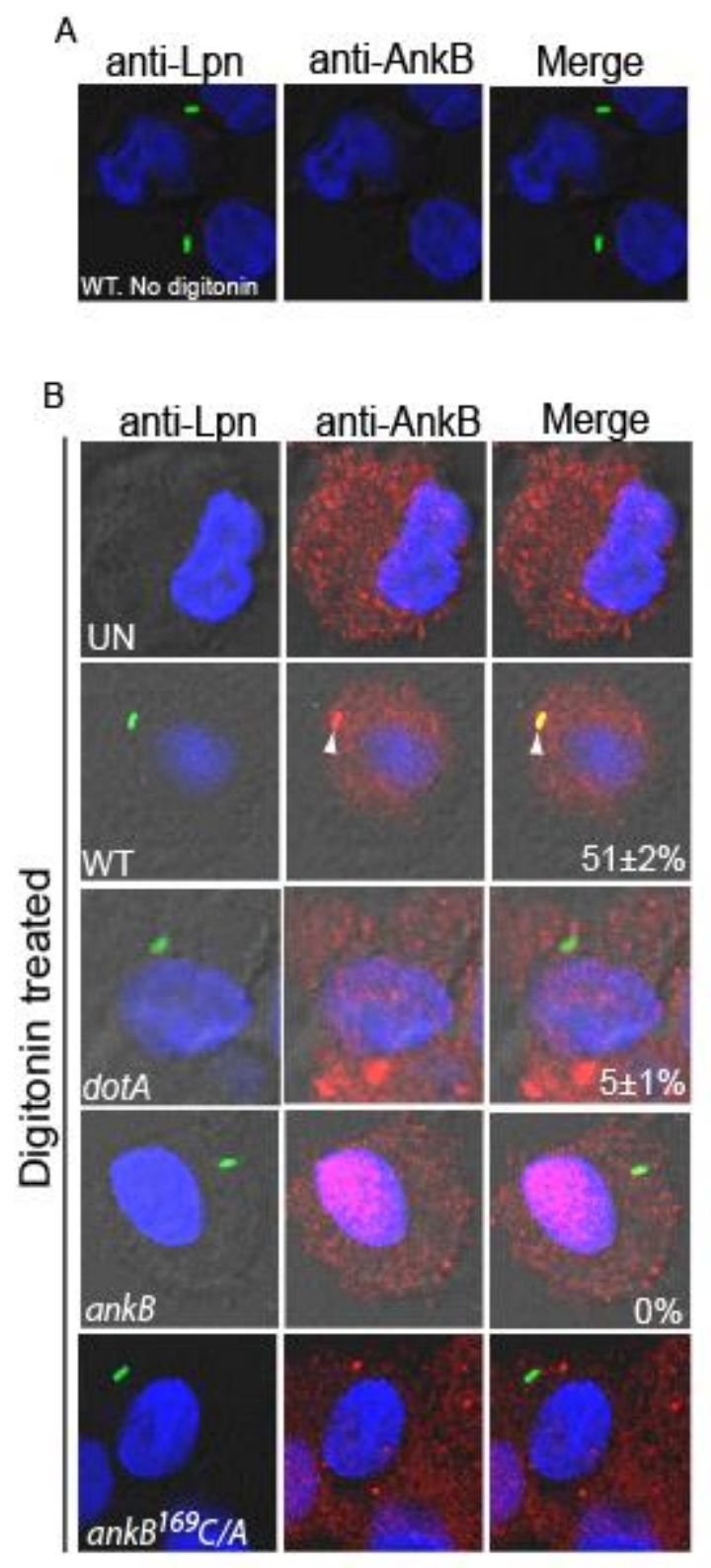

Figure 3: Anchoring of native AnkB injected by attached L. pneumophila to the cytosolic side of the plasma membrane of hMDMs beneath bacterial attachment sites. Representative confocal microscopy images of cytochalasin D-treated infected hMDMs that were preloaded with anti-AnkB antisera (red) in the absence of (A) or presence (B) of digitonin. The hMDMs were infected by L. pneumophila strains (green) for 15 minutes. The numbers in 
the merged images of all panels are mean and standard deviation of the frequency of anchoring AnkB to the plasma membrane beneath attached extracellular bacteria. The data represent analyses of 100 infected cells and are representative of three independent experiments.

\section{Recruitment of the host farnesylation machinery to the plasma membrane beneath attached extracellular L. pneumophila}

Since during infection host-mediated farnesylation of AnkB anchors it to the LCV membrane, and AnkB was exclusively localized beneath bacterial attachment sites, we tested the hypothesis that the host-farnesylation enzymes FTase, IcmT and RCE1 were recruited to the plasma membrane by attached extracellular bacteria to anchor AnkB to the plasma membrane. Cytochalasin D-treated hMDMs were infected with wild-type L. pneumophila and the isogenic mutants $a n k B$ and $\operatorname{dot} A$ at an MOI of 10 for 15 minutes. The cells were then immediately fixed and processed for confocal microscopy to determine if FTase, IcmT and RCE1 were recruited beneath the sites of bacterial attachment. The data showed that FTase, Icm T and RCE1 were all recruited beneath attachment sites of wild-type bacteria at a frequency of $\sim 85 \%$ (Fig. 4A, B, C). In contrast, FTase, Icm T and RCE1 were recruited at a frequency of only $\sim 10 \%$, beneath attachment sites of the $\operatorname{dotA}$ mutant (Student $t$-test, $P<0.006,0.007,0.006$, respectively) (Fig. 4). FTase, IcmT and RCE1 were recruited at a significantly reduced frequency of 43, 47 and $41 \%$, respectively (Student $t$-test, $P<0.01$ ), beneath attachment sites of the ankB mutant bacteria (Fig. 4). The moderate reduction in recruitment of the host enzymes by the ankB mutant is most likely due to the fact that the other $\sim 12$ farnesylated 
effectors of Legionella injected by the ankB mutant [200, 256] interact with the host farnesylation enzymes, while the translocation-defective dotA mutant is severely defective in recruitment of the host enzymes [135]. These data indicate that upon attachment of $L$. pneumophila the Dot/Icm apparatus is essential for recruitment of the host enzymes into the plasma membrane beneath the sites of bacterial attachment. This is the first example of recruitment of the host farnesylation machinery by attached extracellular bacteria to anchor an injected effector to the cytosolic side of the plasma membrane exclusively beneath bacterial attachment sites.

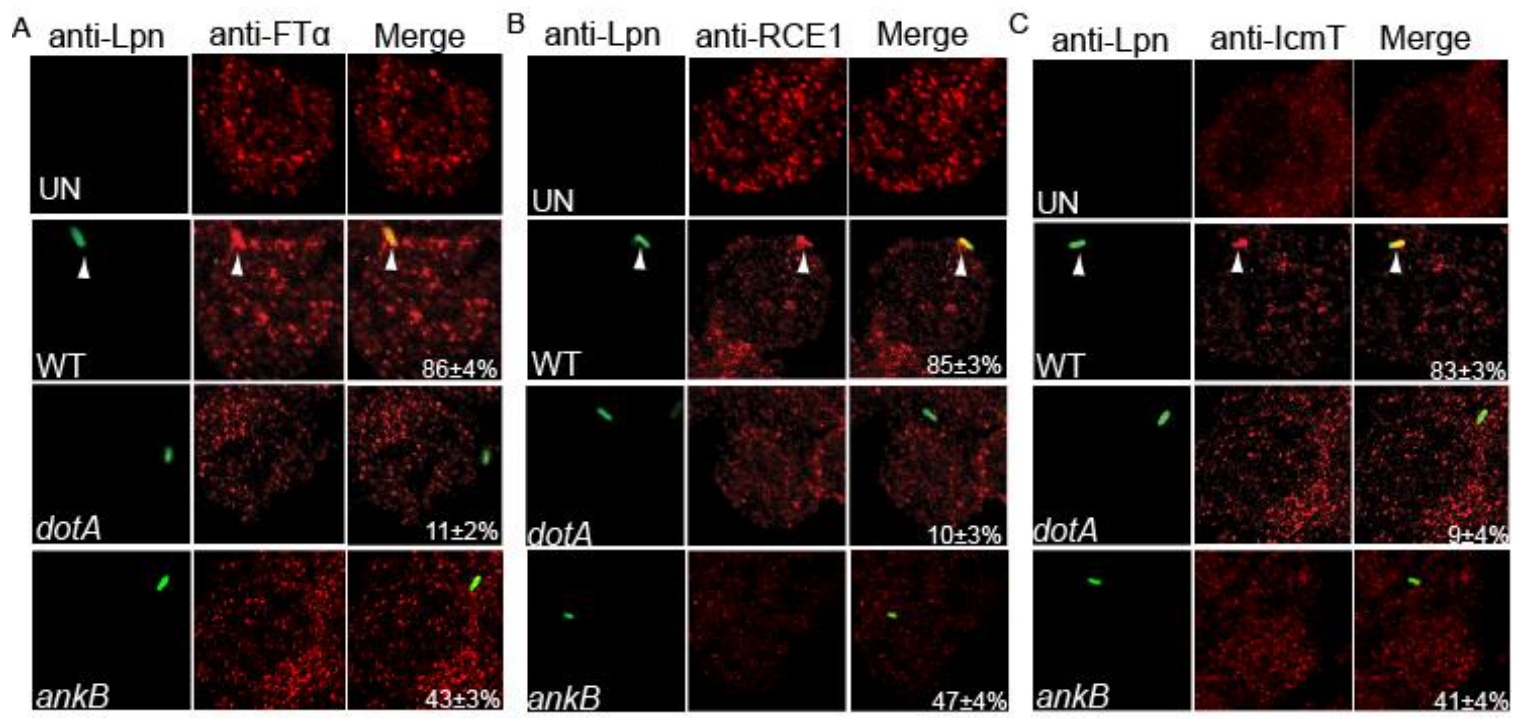

Figure 4: The farnesylation machinery components, FT $\alpha$, RCE1 and IcmT are recruited beneath attachment sites of $L$. pneumophila to hMDMs. The hMDMs pre-treated with cytochalasin D were infected by the wild-type (WT) L. pneumophila and the isogenic dotA or ankB mutants for 15 minutes. Representative confocal microscopy images of infected hMDMs cells showing co-localization of (A) FTa, (B) RCE1 and (C) IcmT proteins to attached WT, $a n k B$ or $\operatorname{dot} A$ bacteria. Bacteria were labeled with anti-Lpn antibody (green) and FT $\alpha$, RCE1 
or IcmT were labeled with the respective specific antibodies (red) and then analyzed by confocal microscopy. The arrowheads indicate intense co-localization of FT $\alpha$, RCE1 or IcmT with the WT strain. The numbers in the merged images of all panels are mean and standard deviation of the frequency of recruitment of FT $\alpha, \mathrm{RCE} 1$ or Icm T beneath attached extracellular bacteria. The data represent analyses of 100 infected cells and are representative of three independent experiments.

\section{Recruitment of the host SCF1 ubiquitin ligase complex beneath attached extracellular \\ L. pneumophila}

During ectopic expression, the AnkB effector interacts with the host Skp1 component of the SCF1 E3-ubiquitin ligase complex, but the location of this interaction during infection is not known [238, 239]. Since AnkB is exclusively localized to the Legionella-containing vacuole (LCV) membrane during infection and to the plasma membrane beneath attached extracellular L. pneumophila, we tested the hypothesis that the SCF1 ubiquitin ligase was recruited to the LCV where it interacts with $\mathrm{AnkB}$ and that this recruitment was rapidly initiated at the plasma membrane beneath bacterial attachment sites. To determine recruitment of SCF1 to the LCV, hMDMs were infected at an MOI of 10 for 1 h with wild-type $L$. pneumophila or the ankB or $\operatorname{dotA}$ translocation-defective isogenic mutant. The data showed that both Skp1 and Cul1 components of the SCF1 were recruited to the LCV of the WT strain at a frequency of $82-84 \%$ (Fig. 5). Recruitment of both host cell components was dependent of a function Dot/Icm translocation system, since the $\operatorname{dot} A$ translocation-defective mutant was severely defective in this recruitment (Fig. 5). To determine whether recruitment of the SCF1 
components was initiated by attached extracellular bacteria, cytochalasin D-treated hMDMs were infected at an MOI of 10 for 15 minutes. Gentamicin treatment of cytochalasin D-treated control cells sterilized the monolayer, which confirms effectiveness of blocking bacterial entry by cytochalasin D (data not shown). The cells were fixed immediately and processed for confocal microscopy. The data showed that Skp1 and Cul1 were recruited beneath bacterial attachment sites at a frequency of $\sim 80 \%$. This recruitment was dependent on a functional Dot/Icm type IV secretion system, since Skp1 and Cul1 were recruited at a frequency of only $\sim 10 \%$ by attached translocation-defective $\operatorname{dot} A$ mutant bacteria (Student $t$-test, $P<0.008$ ) (Fig. 5). Only 34 and $30 \%$ of attached ankB mutant bacteria recruited Skp1 and Cul1, respectively (Fig. 5), which was significantly less than wild-type bacteria (Student $t$-test, $P<0.01$ ). These data show that the Dot/Icm translocation system of L. pneumophila is essential for recruitment of the SCF1 ubiquitin ligase to the LCV and that this recruitment is initiated at the plasma membrane beneath sites of bacterial attachment. This is the first example of recruitment of the host SCF1 to a pathogen-containing vacuole and the initiation of this process at the cytosolic side of the plasma membrane beneath bacterial attachment sites. 
A
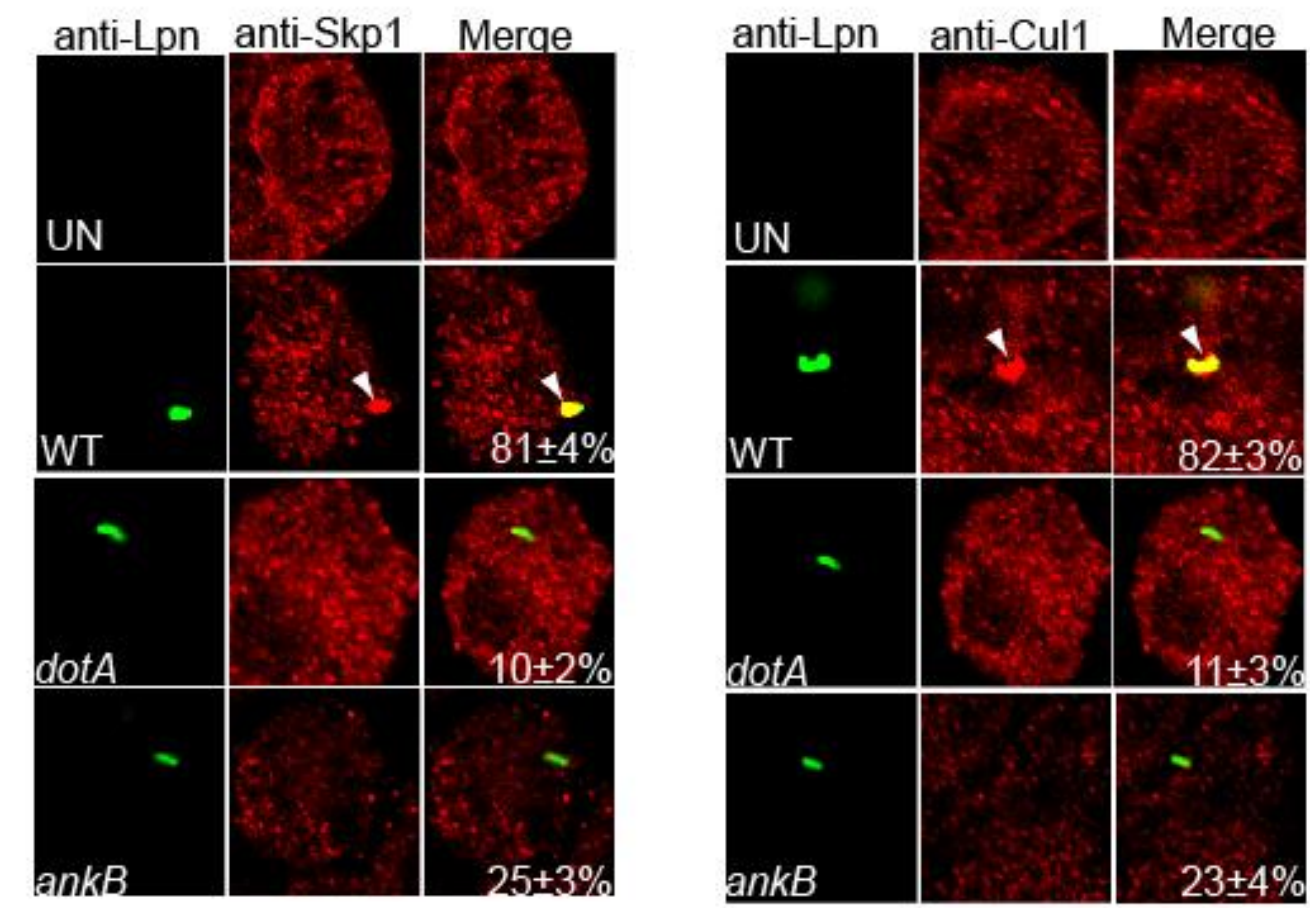

$\mathrm{B}$
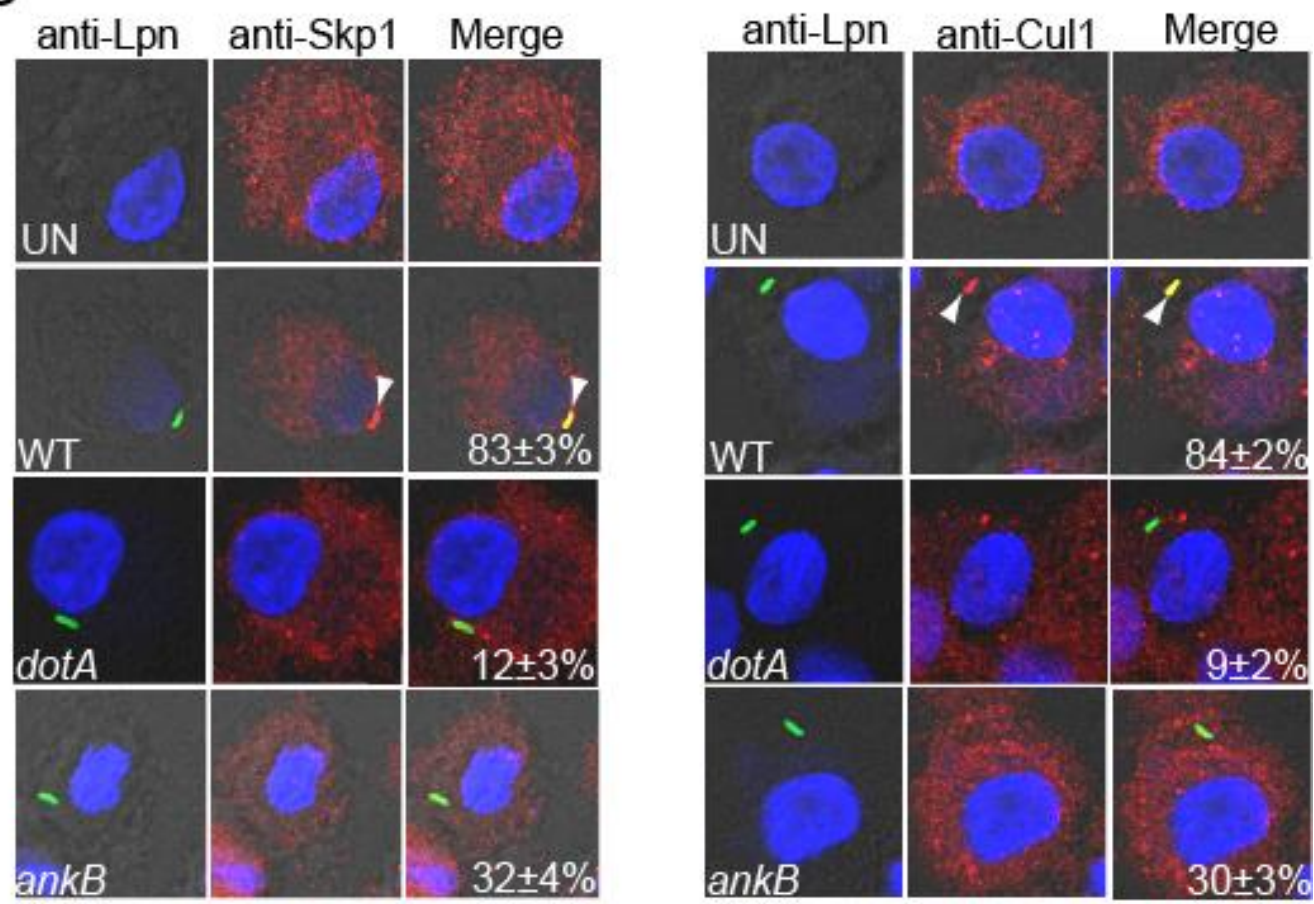

Figure 5: Recruitment of the SCF ubiquitin ligase components Skp1 and Cul1 beneath attachment sites of $L$. pneumophila to hMDMs and the LCV. Untreated (A) or cytochalasin D pre-treated (B) hMDMs were infected by the wild-type (WT) L. pneumophila and the 
isogenic $\operatorname{dotA}$ or ankB mutants for 2 hours (A) or 15 minutes (B), followed by fixation and processing for confocal microscopy. Representative confocal microscopy images of infected hMDMs cells show co-localization of Skp1 and Cul1 proteins (red) with the LCV (green) (A) and with attached extracellular L. pneumophila (B). The arrowheads indicate intense colocalization of Skp1 or Cul1 with the WT bacteria. The numbers in the merged images of all panels are quantification of the frequency of recruitment of Skp1 or Cul1 beneath attached extracellular bacteria. The data represent analyses of 100 infected cells and are representative of three independent experiments.

\section{Elevated levels of cellular amino acids triggered by attached extracellular $L$. pneumophila}

The ultimate function of the LCV membrane-anchored AnkB effector is to generate high levels of cellular amino acids through host proteasomal degradation of $\mathrm{K}^{48}$-linked polyubiquitinated proteins [86]. Therefore, we determined whether the injected AnkB by attached extracellular L. pneumophila resulted in elevated levels of cellular amino acids through degradation of the polyubiquitinated proteins assembled beneath bacterial attachment sites [238]. To achieve this, cytochalasin D-treated hMDMs were infected by the wild-type strain or the isogenic ankB mutant L. pneumophila. Trypan blue straining of the cells showed that there was no detectable effect of cytochalasin $\mathrm{D}$ on permeability of the plasma membrane (data not shown). The hMDMs were lysed and the relative levels of free amino acid were

determined by GC-MS. The data showed that attached wild-type L. pneumophila triggered a rapid rise in the levels of amino acids, relative to uninfected cells (Student $t$-test, $p<0.001$ ) 
(Fig. 6). In contrast, attachment of the ankB mutant bacteria to hMDMs did not alter cellular levels of amino acids relative to uninfected cells (Fig. 6). A detailed working model of AnkBdependent nutritional preparation of the host cell is shown, which depicts the translocation, rapid farnesylation and polyubiquitination of the AnkB targets directly beneath the attachment sites of extracellular bacteria (Fig. 7). Thus, translocation of AnkB by attached extracellular $L$. pneumophila results in increased levels of cellular amino acids, which are needed to block a potential starvation response and differentiation of $L$. pneumophila into the non-replicative phase (Fig. 7). This is the first example of a strategy by an intracellular pathogen to trigger rapid nutritional remodeling of the host cell upon attachment to the plasma membrane, and as a result, a gratuitous surplus of cellular amino acids is generated to support proliferation of the incoming pathogen. In addition, the AnkB-dependent increase in free cellular amino acids can be achieved within 15 minutes of extracellular bacterial attachment. 


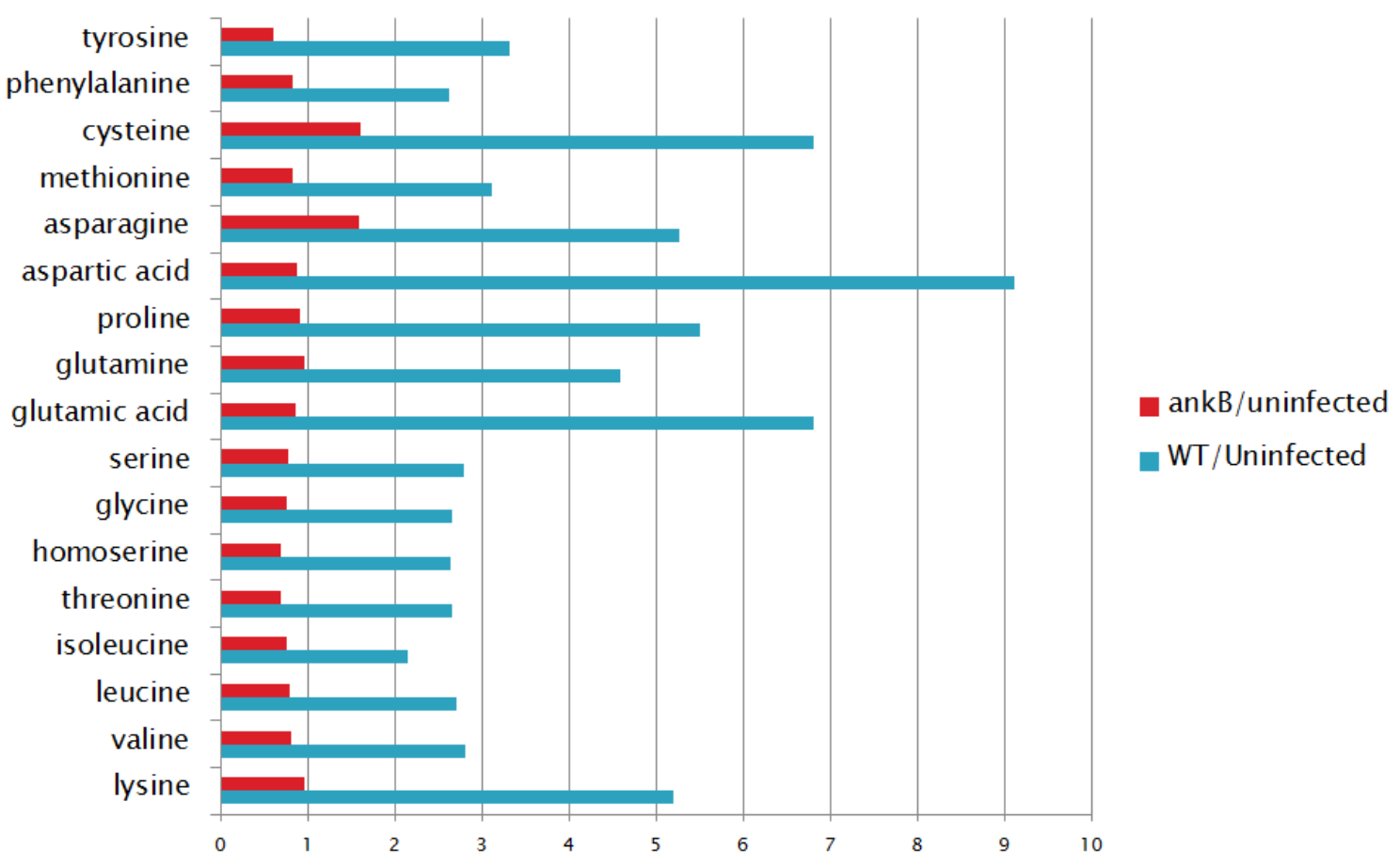

Fold Ratio of Amino Acid Levels

Figure 6: Intimate attachment of $L$. pneumophila to hMDMs triggers an increase in cellular levels of free amino acids. The hMDMs pre-treated with cytochalasin D were infected for $15 \mathrm{~min}$, followed by preparation of cellular lysates and determination of the relative levels of cellular amino acids by GC-MS. Amino acid levels are expressed as the fold ratio of infected/uninfected hMDMs. The analyses were performed in triplicates, and the data shown are one of three representative experiments. The data represent analyses of 100 infected cells and are representative of three independent experiments. 


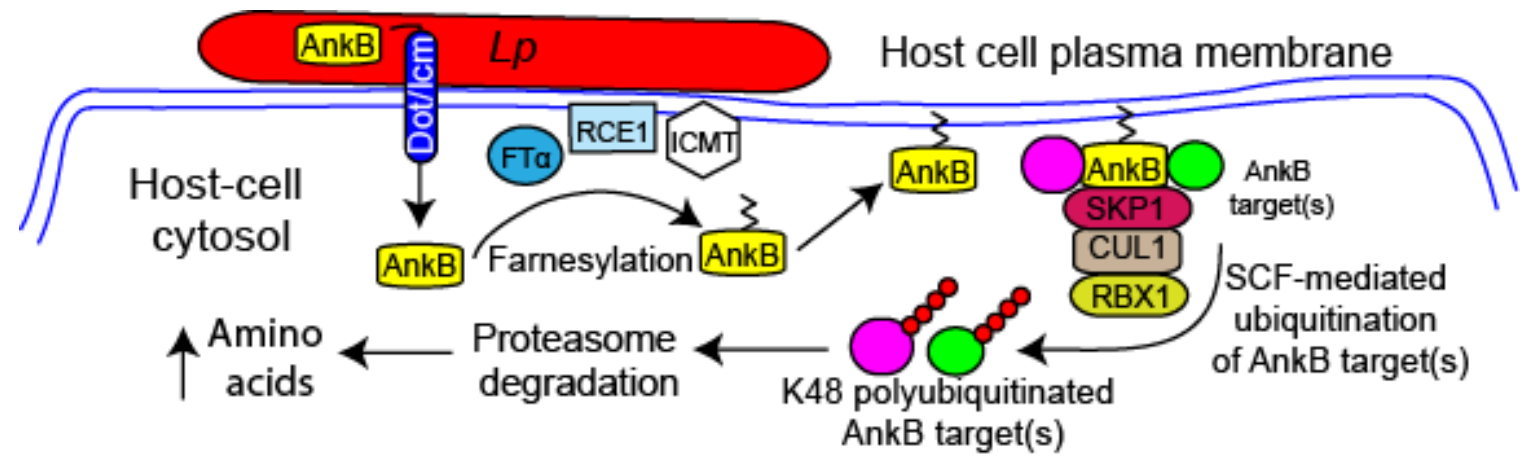

Figure 7: A working model of AnkB-mediated nutritional preparation of the host cell

by attached extracellular $L$. pneumophila. Upon bacterial attachment, the Dot/Icm

translocation system is triggered to inject bacterial effectors, one of which is AnkB. The host farnesylation enzymes are recruited beneath bacterial attachment sites and are essential for farnesylation of AnkB, which enables anchoring this effector to the cytosolic side of the plasma membrane beneath bacterial attachment sites. The host SCF1 ubiquitin ligase complex is recruited to the plasma membrane where it interacts with the F-box domain of AnkB to trigger assembly of $\mathrm{K}^{48}$-linked polyubiquitinated proteins beneath bacterial attachment sites. Proteasomal degradation of the $\mathrm{K}^{48}$-linked polyubiquitinated proteins generates higher levels of cellular amino acids, particularly the limiting ones, such as cysteine, which is a metabolically preferable source of carbon and energy for $L$. pneumophila. The availability of higher levels of cell amino acids upon entry of $L$. pneumophila circumvents the entering bacterium from a potential starvation response and differentiation into the motile non-replicative phase. The elevated cellular levels of amino acids trigger differentiation of L. pneumophila into the replicative phase and are main sources of carbon and energy that feed the TCA cycle to power intracellular bacterial proliferation. 


\section{Discussion Section 1}

L. pneumophila has acquired a large array of eukaryotic-like proteins possibly through horizontal gene transfer through evolution with protozoan host $[137,138]$. While $\sim 300$ effectors are translocated through the Dot/Icm type IVB secretion system, intracellular replication of L. pneumophila strain AA100/130b and the Paris stain is dependent upon the bona fide F-box effector AnkB [131, 239]. This unique ability for a single effector to dictate the fate of intracellular survival shows the importance to characterize the function of AnkB during infection. L. pneumophila utilizes AnkB, which interacts with the SCF1 E3 ubiquitin ligase complex to trigger $\mathrm{K}^{48}$-linked polyubiquitination that are targeted for proteasomal degradation. This increases the levels of free cellular amino acids above the threshold needed for intracellular replication [86]. This increase in free cellular amino acids is crucial to prevent a starvation response and differentiation into the non-replicative phase [86]. When the host cell proteasomes are inhibited WT L. pneumophila undergoes a starvation response, similar to the ankB mutant strain, that can be reversed through the addition of amino acids into the cell culture medium [86].We have shown AnkB is translocated from attached extracellular bacteria, where it anchors directly beneath attached extracellular bacteria through host-mediated farnesylation of the CAAX motif in the C-terminus of AnkB [241]. The $\mathrm{K}^{48}$-linked polyubiquitinated proteins surrounding the LCV have been observed throughout the infection, therefore unlike some Dot/Icm effectors such as RalF and SidC, AnkB is functional throughout the infection [257, 258].

The translocation of AnkB from attached bacteria shows a necessity for the immediate increase of amino acid sources, which provides the carbon and energy required for 
intracellular replication. While it is not uncommon for L. pneumophila effectors to be translocated from attached extracellular bacteria, this has only been shown for a relatively small number of the $\sim 300$ effectors [258]. Interestingly, AnkB is the only ankyrin effector shown to be translocated from attached bacteria when seven L. pneumophila ankyrin effectors were analyzed [176]. It is possible that L. pneumophila translocates a subpopulation of the effectors upon bacterial attachment that are needed for critical aspects of infection, such as early LCV maturation and nutrient acquisition. Due to the biphasic life cycle of $L$. pneumophila, which uses nutrient deprivation as a major signal for switching from the replicative phase to the transmissive phase, the immediate AnkB-dependent increase in free cellular amino acids can be easily justified. When the AnkH and AnkJ effectors are deleted, there is a slight defect in intracellular replication, however neither are translocated upon attachment [176]. Future studies investigating effector translocation kinetics will provide crucial details to how L. pneumophila determines when certain effectors are translocated.

The host cell ubiquitination and farnesylation machinery are recruited within 15 minutes to the sites of extracellular bacterial attachment. While AnkB is the only CaaX motif containing effector shown to be translocated upon bacterial attachment, some of the other CaaX motif containing effectors are also probably translocated upon attachment, since $\sim 40 \%$ of the ankB mutant strain attached extracellular bacteria recruit the host cell farnesylation machinery enzymes FT $\alpha$, RCE1 and ICMT. The recruitment of host farnesylation machinery is critical for the function of AnkB, since farnesylation of AnkB is required in order to anchor into the cytosolic face of the LCV membrane [135] and to function as a platform for the recruitment of $\mathrm{K}^{48}$-linked polyubiquitinated proteins to the LCV. L. pneumophila is the only intracellular pathogen known to recruit the farnesylation enzymes to the point of 
extracellular bacterial attachment, as well as to the LCV, to allow anchoring of bacterial effector proteins. Interestingly, the Salmonella effectors Ssel and $\mathrm{SspH} 2$ are S-palmitoylated, through the addition of a lipid moiety to the effector, which enables the effector to alter its sub-cellular localization by anchoring into the plasma membrane [259]. Similar to AnkB, this host-mediated post-translational lipidation is required for the function of the Salmonella protein in vivo $[135,259]$. This shows a unique ability of a pathogen to exploit the host cell by manipulating the farnesylation machinery for modification of bacterial effector proteins. Further investigation into effectors that are translocated from attached extracellular bacteria could prove valuable to determine host cellular processes that are manipulated from attached bacteria that could benefit the infection.

While there are seven L. pneumophila effectors harboring the eukaryotic CaaX motif, there are only five F-box proteins in L. pneumophila (Lpg2525, LicA, PpgA, LegU1 and LegAU13 which is the AnkB homologue in the Philadelphia L. pneumophila strain) [131, 228]. Three of these are bona fide F-box proteins (LicA,AnkB LegU1) that interact with the Skp1 component of the SCF1 E3 ubiquitin ligase complex, while the predicted F-box motifs identified in PpgA and Lpg2525 may not be functional in the eukaryotic hosts, as they do not bind Skp1 [228]. Therefore, in addition to AnkB, L. pneumophila has acquired 3 additional functional F-box proteins that likely ubiquitinate other host proteins in a proteasomal dependent manner similar to AnkB [228]. The F-box protein LegU1 has been shown to ubiquitinate the eukaryotic protein BAT3 [228], but the effect of this ubiquitination is not known. Importantly, unlike AnkB in the AA100/130b strain and the Paris which is required for $\mathrm{LCV}$ polyubiquitination and intracellular replication, the homologue to AnkB in the Philadelphia strain LegAU13 does not cause polyubiquitination of the LCV and is not 
required for intracellular replication $[131,228,239,260]$. Therefore, the genetic diversity within $a n k B$ demonstrates the capability of L. pneumophila to survive intracellularly through several different mechanisms not shared among the strains. Determining the targets of AnkB in each of the strains could provide a solution for the phenotypic differences observed among the L. pneumophila strains. It is possible that the substrates of AnkB are different among the strains and specific substrates recognized by AnkB in the AA100/130b strain and the Paris strain provide the amino acids needed to support intracellular replication. L. pneumophila is auxotrophic for seven amino acids, indicating the preference and importance of obtaining host amino acids during the infection. In the Philadelphia strain of L. pneumophila the LCV is polyubiquitinated by the effector SidC, however the consequence of this polyubiquitination is not known [261].

Similar to the farnesylation enzymes, the Skp1, Cul1 and Rbx1 components of the SCF1 E3 ubiquitin ligase complex are cytosolic enzymes in mammalian cells [218]. The recruitment of the SCF1 complex to the plasma membrane beneath extracellular bacteria most likely results from the strong interaction the F-box proteins with the cytosolic Skp1 enzyme. This highlights the first example of the host cell ubiquitination machinery being recruited to the site of attachment of extracellular bacteria to provide a function critical to the subsequent intracellular survival of L. pneumophila.

Taken together, the recruitment of the eukaryotic farnesylation and ubiquitination machineries to the point of extracellular bacterial attachment show a unique ability of an intracellular pathogen to manipulate host cell machineries to provide altered sub-cellular localization of bacterial effector proteins. The strategies are utilized by L. pneumophila to create a surplus of amino acids and prevent a starvation response. Further research will 
probably lend evidence to other intracellular pathogens to trigger a surplus of host cell nutrients in order to support the high energy demands needed for intracellular replication. 
Results Section 2. The complete and ubiquitinated proteome of the Legionella-containing vacuole within human macrophages.

\section{Purification of the LCV from human macrophages}

Due to the requirement of lysine ${ }^{48}$-linked polyubiquitination of the LCV in the AA100/130b L. pneumophila strain, we sought to determine the identity of the ubiquitinated proteins localized to the LCV. Some of these ubiquitinated proteins localized to the WT strain LCV are possible targets of the F-box effector protein AnkB which acts as the scaffold protein to cause $1 y \operatorname{sine}^{48}$-linked polyubiquitination of its ankyrin domain substrates on the LCV. To determine the identity of the ubiquitinated proteome, LCV's must be isolated from the WT strain and ankB mutant strain, therefore we sought to determine the identity of all host proteins on the LCV in addition to the ubiquitinated proteome. In addition, this work will provide the first proteome of the LCV isolated from human macrophages, the only known mammalian host.

U937 human macrophages were infected with L. pneumophila WT strain AA100/130b or its isogenic ankB mutant strain. The ankB mutant strain evades lysosomal fusion and is localized within an ER-derived LCV, similar to the WT strain [131]. However, the ankB mutant strain fails to replicate within human macrophages and amoeba due to the levels of cellular amino acids being below the threshold needed as the major source of carbon and energy to support intra-vacuolar proliferation of $L$.

pneumophila [86, 250, 262]. The U937 human macrophage cell line is widely used in studies on Legionella-human macrophage interaction and was used in this study instead of primary human monocytes due to the need of a large number of cells to isolate LCVs, 
since only $\sim 20 \%$ of the cells become infected. Following a $4 \mathrm{~h}$ infection of $6 \times 10^{8} \mathrm{U} 937$ macrophages, LCVs were purified according to previously reported protocols with minor modifications $[147,263]$. Cells were lysed through dounce homogenization and the postnuclear supernatant (PNS) was used to isolate LCVs through density ultracentrifugation on a discontinuous sucrose gradient which resulted in purified LCVs at the 55-65\% interface (Fig. 8A). To ensure vacuole integrity following purification, isolated LCVs were evaluated using confocal microscopy after differential membrane permeabilization as well as vacuole marker staining to ensure the LCV membranes were intact (Fig. 8 BC). Vacuoles were labeled with a polyclonal anti-Legionella antibody prior to vacuolar permeabilization, which resulted in $\sim 20 \%$ of bacteria being labeled (data not shown), while $100 \%$ of bacteria were labeled after vacuolar membrane permeabilization, indicating the LCV membrane is intact on $\sim 80 \%$ of the isolated LCVs (Fig. 8B). We next evaluated the presence of polyubiquitinated proteins, which showed that $\sim 70 \%$ of isolated LCVs were decorated with polyubiquitinated proteins (Fig. 8C). Isolated LCVs were solubilized in $1 \%$ Triton X-100 and the eukaryotic proteins associated with the LCV were identified by high throughput Liquid Chromatography coupled with tandem Mass Spectrometry (LC-MS). The MS was loaded with $2 \mu \mathrm{g}$ of protein for each LCV sample. The proteome was obtained from analyses of two LCV preparations of the WT strain and the ankB mutant, and only the proteins that were reproducible in both preparations were included in our analyses. A positive protein identification for the proteome was based on at least two unique peptides. Although numerous proteins were identified, it is likely that some proteins present with scarce quantities on the LCV are not detectable by our analyses. 
A

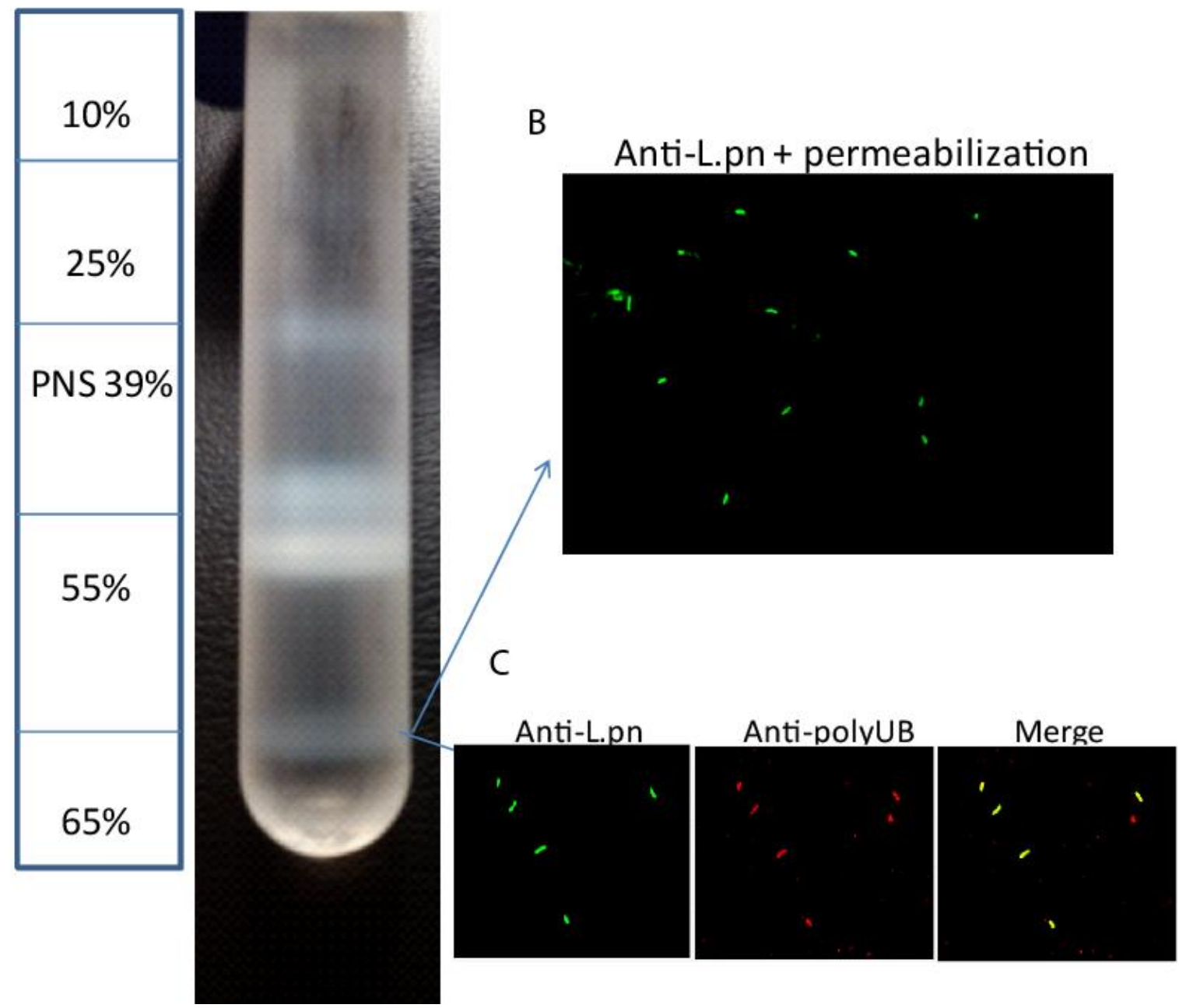

Fig. 8. LCV purification using a discontinuous sucrose gradient. U937 macrophages

were infected with WT L. pneumophila or the isogenic ankB strain at an MOI of 50 for 30 minutes washed and the infection proceeded for 4 hours. Cells were lysed through dounce homogenization and the post-nuclear supernatant was used to isolate LCVs through density ultracentrifugation on a discontinuous sucrose gradient. A) Diagram of the sucrose gradient showing the isolated LCVs at 55-65\% interface and B) confocal microscopy of isolated LCVs labeled with mouse anti-L. pneumophila following vacuole 
membrane permeabilization. C) Confocal microscopy of isolated LCVs labeled with rabbit anti-L. pneumophila antiserum and mouse anti-polyubiquitin antibodies.

\section{The Total LCV Proteome}

The proteome of WT strain AA100/130b LCV contained 1,193 eukaryotic proteins, while the LCV of the ankB mutant strain contained 1,546 eukaryotic proteins (Supp Tables 1 and 2 that are found in Bruckert et al. Journal of Proteome Research) [264]. We profiled the proteins according to various cellular functions, including transcription/translation, vesicle trafficking, immune response, ribosomal proteins, ubiquitination, proteasome machinery components, signaling, cytoskeleton arrangement, and metabolism (Fig. 9 and 10). Using these categories we identified the largest proportion of WT strain LCV proteins to be involved in metabolism (21\%) while cytoskeleton arrangement (12\%), signaling (12\%) and transcription/translation (11\%) were highly represented (Fig. 9). Proteins involved in immune response (8\%), ribosome machinery $(6 \%)$, vesicle trafficking $(6 \%)$ and ubiquitin-dependent proteolysis $(6 \%)$ were significantly ( $\mathrm{p}$ value $\leq 0.05$ ) represented among the WT strain LCV proteome (Fig. 9). Despite the presence of additional proteins on the ankB mutant LCV, the overall distribution of the proteins based on cellular function were very similar to the WT strain LCV proteome (Fig. 9 and 10). The large numbers of shared proteins on isolated LCVs of WT strain and the ankB mutant were consistent with the findings that the ankB mutant strain is localized in an ER-derived LCV that evades lysosomal fusion [131] but lacks 
sufficient levels of amino acids for intra-vacuolar proliferation [86]. The ankB mutant LCV contained $80 \%$ of the proteins on the WT strain LCV with an additional 354 proteins primarily involved in regulation and initiation of translation, transcription, apoptosis, and immune response signaling. It is likely that degradation of lysine ${ }^{48}$-linked polyubiquitinated proteins on the WT strain LCV renders them undetectable by proteomic analysis. The degradation of lysine ${ }^{48}$-linked polyubiquitinated proteins on the WT strain LCV also alters the relative abundance of identifiable proteins in the proteome of the two strains. However, it is unknown if any of the additional 354 proteins on the ankB mutant strain LCV are the targets of AnkB.

Proteome data of the WT strain LCV regarding metabolism identified proteins involved in neutral amino acid transport (SLC1A5, SLC38A2, SLC3A2), cationic amino acid transport (SLC7A1) and monocarboxylate transport (SLC16A1, SLC16A3) as well as a variety of ATPases involved in calcium, sodium and potassium transport. A large proportion of metabolism related proteins are involved in carbohydrate metabolism, especially glycolysis or glucose transport (7\%), while 5\% are involved in lipid metabolism. Both the ankB mutant and WT strain LCV proteomes harbor phosphatidylinositol phosphatases (INPP5D, INPPL1), kinases (PI4KA), phosphatidylinositol 3,4,5-trisphosphate-dependent GTPase-activating proteins (ARAP1, ASAP1) and guanine exchange factors (SWAP70, PREX1, DEF6) (Supp Tables 1 and 2 that are found in Bruckert et al. Journal of Proteome Research) [264]. There are also proteins that bind to membranes or vesicles enriched in phosphatidylinositol that function in early endosomal trafficking (CLINT1, RUFY1) and receptor-mediated endocytosis (EPN1). Consistent with the findings that the LCV of the WT strain and the ankB mutant 
strain is RER-derived, the proteome data revealed 70-80 (6\%) 40S and 60S ribosomal associated proteins present on WT and $a n k B$ mutant LCVs (Fig. 2 and 3). Our data identified 6-7\% of the WT and $a n k B$ mutant LCV proteomes play a role in vesicular trafficking, which included 8 small Rab GTPases (Rab1, Rab2, Rab5, Rab6, Rab7, Rab10, Rab11, Rab14) as well as 4 sorting nexins and multiple ADP ribosylation factors involved in endocytic recycling, vesicle transport and vesicle budding. The ankB mutant LCV had 4 additional Rab GTPases (Rab8, Rab13, Rab27 and Rab35) (Supp Tables 1 and 2 that are found in Bruckert et al. Journal of Proteome Research) [264]. The high similarity in ribosomal and vesicular trafficking proteins in WT and ankB mutant LCV proteomes is very consistent with the findings on biogenesis of the ankB mutant LCV is very similar to the WT strain [131].

Proteins involved in apoptosis and immune responses, which comprise $8 \%$ of the WT strain LCV proteome, were also identified. These include multiple major histocompatibility complex proteins, ligands and receptors involved in T cell adhesion (intercellular adhesion molecule 1, 3) and signaling (leukocyte-associated immunoglobulin-like receptor 1, HCLS1 binding protein 3), promoters of cell apoptosis (BCL2-associated athanogene 6, caspase recruitment domain family member 6), inhibitors of apoptosis (defender against cell death 1, FAM129B) and TNF- $\alpha$ regulators and proteins, which promote LPS-induced TNF- $\alpha$ production (thymocyte selection associated family member 2). The proteome of the ankB mutant LCV contained 84 of the 92 immune response proteins on the WT strain LCV with an additional 42 proteins. These include 7 cluster of differentiation molecules (CD109, CD47, CD58, CD63, CD82, CD83, CD84), 2 BCL2 associated proteins (BAG3, BAX) and 2 negative regulators of 
apoptosis (TNFAIP8 and AVEN) (Supp Tables 1 and 2 that are found in Bruckert et al. Journal of Proteome Research) [264].

Proteins involved in cellular signaling pathways on the WT strain and the ankB mutant LCV include tyrosine-protein kinases that play a role in the response to environmental stress and cytokines such as TNF- $\alpha$ (MAP4), regulation of cell growth, differentiation, migration and the immune response (CSK), cytoskeleton remodeling in response to extracellular stimuli, cell motility and receptor endocytosis (ABL2, ARAP1). Additional signaling proteins included Rho GTPase activating proteins (ARHGAP1, ARHGAP17, ARHGAP18), Rab GTPase activating proteins (TBC1D10B, TBC1D15, TBC1D5) and guanine nucleotide-binding proteins (G proteins).

The WT strain and ankB mutant LCV proteomes contained proteins involved in cytoskeletal membrane integrity and organization (actin, coronin) and proteins that regulate actin and microtubule polymerization (ARP2/3 complex, KANK1, LASP1,). Interestingly, molecular chaperones that play a role in the folding of actin and tubulin (CCT2-CCT8) were present on the LCV, possibly indicating following initial LCV formation various cytoskeleton proteins are constantly recruited to the LCV membrane [265].

Validation of many of the proteins detected in the proteome of the LCV in our analyses comes from published studies that showed that at least 17 of the proteins identified in our proteome have been already shown to be localized to the LCV using different strategies, such as confocal microscopy $[74,79,135,147,237]$. The WT strain and ankB mutant LCV contain a significant portion (6\%) of proteins involved in ubiquitination and proteasomal degradation (Fig. 9 and 10). In agreement with findings of 
colocalization of farnesyltransferase $\alpha$ [135], SKP1 [241] and p97 [237] with the LCV by confocal microscopy, our proteome data identified the SKP1 component of the SCF E3 ubiquitin ligase complex and the ATPase p97 on the LCV. In addition, both proteomes contained RAD23A and RAD23B which serve as multiubiquitin chain receptors that bind to the $26 \mathrm{~S}$ proteasome and delivers $\mathrm{K}^{48}$-linked polyubiquitinated proteins for proteasomal degradation. Four E2 ubiquitin conjugating enzymes and eight E3 ubiquitin ligases were identified on the WT strain LCV (Table 1). The E3 ubiquitin ligases identified on the WT strain LCV regulate apoptosis, NF- $\kappa \beta$ activation and IFN- $\beta$ production [266, 267]; while the E2 ubiquitin conjugation enzymes catalyze the synthesis of $\mathrm{K}^{48}$ and $\mathrm{K}^{63}$-linked polyubiquitin chains $[260,268]$ (Table 1). The eight ubiquitin specific peptidases on the WT strain LCV can act as deubiquitinases (VCPIP1(p97), USP14, USP15, USP9X), that are able to remove ubiquitin moieties from polyubiquitin chains, which prevents proteasomal degradation, or are involved in lysine ${ }^{48}$-linked polyubiquitination disassembly (USP5) (Table 1). The ankB mutant LCV contained nine E3 ubiquitin ligases of which three were found on the WT strain LCV (Table 2). The E3 ubiquitin ligases on the ankB mutant LCV are involved in regulation of DNA, p53 activation and mTORC1 signaling pathway [269, 270]. The ankB mutant LCV contained four additional ubiquitin-conjugating enzymes including two atypical ubiquitin conjugating enzymes (UBE2H, UBE2O) and UBE2M, which catalyze the attachment of the ubiquitin like protein NEDD8 onto other proteins (Table 2). 


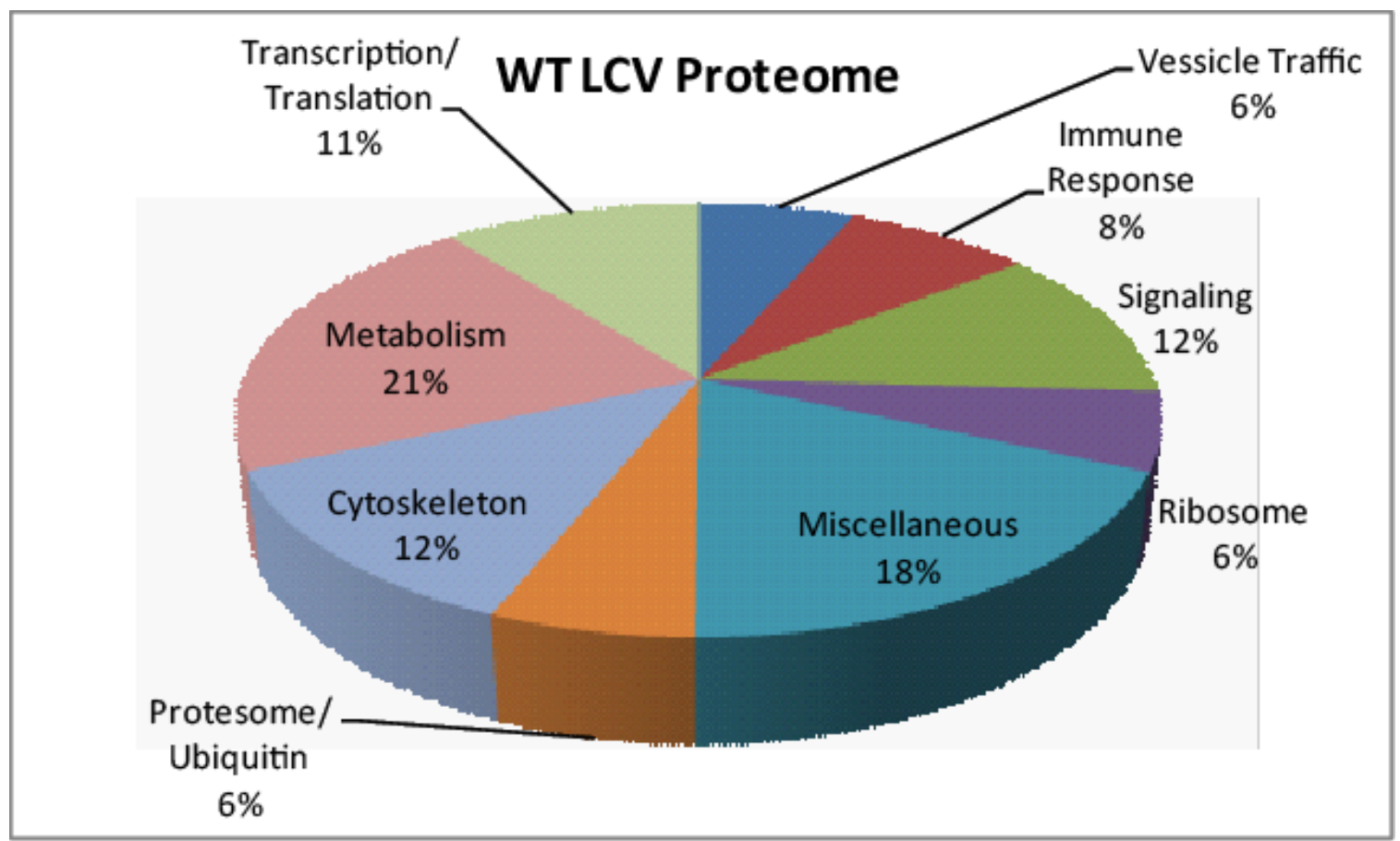

Fig. 9. Functional classification of eukaryotic proteins localized to the WT strain

LCV. The 1193 eukaryotic proteins localized to the WT strain LCV identified by High Throughput LC/MS were grouped according to their cellular function according to the UniProt and GeneCards database sets. 


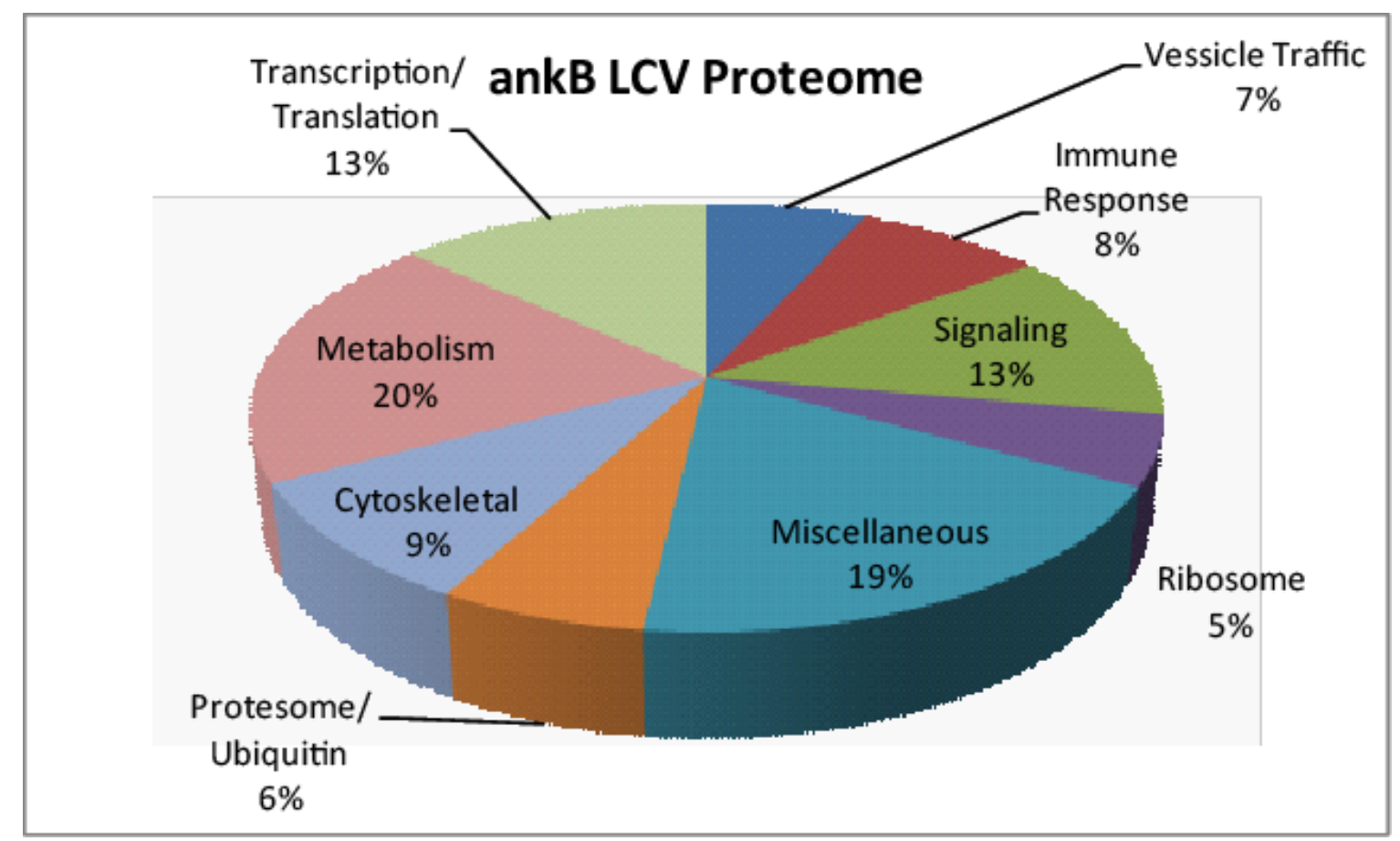

Fig. 10. Functional classification of eukaryotic proteins localized to the ankB mutant strain LCV. The 1546 localized to the ankB mutant LCV identified by High Throughput LC/MS were grouped according to their cellular function according to the UniProt and GeneCards database sets. 
Table 1. Ubiquitin-conjugation and degradation enzyme WT LCV

E3 ubiquitin ligase

ARIH1 Ariadne RBR E3 ubiquitin protein ligase 1

HERC1 E3 ubiquitin protein ligase HERC1

HUWE1 E3 ubiquitin protein ligase HUWEI

NEDD4 E3 ubiquitin-protein ligase NEDD4

RNF31 ring finger protein 31

TRIM21 tripartite motif containing 21

UBR4 ubiquitin protein ligase E3 component $\mathrm{n}$-recognin 4

E2 ubiqutin-conjugating enzymes

UBE2K ubiquitin-conjugating enzyme E2K

UBE2L3 ubiquitin-conjugating enzyme E2L3

UBE2N ubiquitin-conjugating enzyme E2N

UBE2V1 ubiquitin-conjugating enzyme E2

variant 1

Ubiquitin specific peptidase

USP10 ubiquitin specific peptidase 10

USP14 ubiquitin specific peptidase 14

USP15 ubiquitin specific peptidase 15

USP47 ubiquitin specific peptidase 47

USP5 ubiquitin specific peptidase 5

USP8 ubiquitin specific peptidase 8

USP9X ubiquitin specific peptidase 9

VCP valosin containing protein $(p 97)$ 
Table 2. Ubiquitin-conjugation and degradation enzymes

\begin{tabular}{|c|c|}
\hline \multicolumn{2}{|c|}{ ankB LCV } \\
\hline \multicolumn{2}{|c|}{ E3 ubiquitin ligase } \\
\hline \multirow[t]{2}{*}{ ARIH 2} & Ariadne RBR E3 ubiquitin protein \\
\hline & ligase 2 \\
\hline \multirow[t]{2}{*}{ BIRC6 } & Baculoviral IAP repeat \\
\hline & containing 6 \\
\hline HUWE1 & E3 ubiquitin protein ligase HUWEI \\
\hline \multirow[t]{2}{*}{ HECTD3 } & HECT domain containing E3 \\
\hline & ubiquitin protein ligase 3 \\
\hline \multirow[t]{2}{*}{ MYCBP2 } & MYC binding protein 2, E3 \\
\hline & ubiquitin protein ligase \\
\hline RNF130 & ring finger protein 130 \\
\hline TRIP12 & E3 ubiquitin protein ligase TRIP12 \\
\hline \multirow[t]{2}{*}{ UBR4 } & ubiquitin protein ligase E3 \\
\hline & component n-recognin 4 \\
\hline \multirow[t]{2}{*}{ UBR5 } & ubiquitin protein ligase E3 \\
\hline & component n-recognin 5 \\
\hline \multicolumn{2}{|c|}{ E2 ubiqutin-conjugating enzymes } \\
\hline \multicolumn{2}{|c|}{ UBE2H ubiquitin-conjugating enzyme E2H } \\
\hline \multicolumn{2}{|c|}{ UBE2J1 ubiquitin-conjugating enzyme E2J1 } \\
\hline \multicolumn{2}{|c|}{ UBE2K ubiquitin-conjugating enzyme E2K } \\
\hline \multicolumn{2}{|c|}{ UBE2L3 ubiquitin-conjugating enzyme E2L3 } \\
\hline \multicolumn{2}{|c|}{ UBE2M ubiquitin-conjugating enzyme E2M } \\
\hline \multicolumn{2}{|r|}{ tin-conjugating enzyme E2N } \\
\hline \multicolumn{2}{|c|}{ UBE2O ubiquitin-conjugating enzyme $E$} \\
\hline \multicolumn{2}{|c|}{ UBE2V1 ubiquitin-conju } \\
\hline \multicolumn{2}{|r|}{ E2 variant 1} \\
\hline \multicolumn{2}{|c|}{ Ubiquitin specific peptidase } \\
\hline USP10 & ubiquitin specific peptidase 10 \\
\hline USP14 & ubiquitin specific peptidase 14 \\
\hline USP15 & ubiquitin specific peptidase 15 \\
\hline USP19 & ubiquitin specific peptidase 19 \\
\hline USP24 & ubiquitin specific peptidase 24 \\
\hline USP34 & ubiquitin specific peptidase 34 \\
\hline USP47 & ubiquitin specific peptidase 47 \\
\hline USP5 & ubiquitin specific peptidase 5 \\
\hline USP8 & ubiquitin specific peptidase 8 \\
\hline USP9X & ubiquitin specific peptidase 9 \\
\hline VCP & valosin containing protein ( $p 97)$ \\
\hline
\end{tabular}




\section{MetaCore Analysis of the total LCV proteome}

Bioinformatic analysis of the LCV proteome was performed using MetaCore (Thomson Reuters) [248, 249]. MetaCore software determined the most significant relationships among pathway maps, process networks, GO processes and metabolomic pathways from eukaryotic proteins localized to the LCV. The top pathway maps identified in WT and $a n k B$ mutant LCV proteomes include regulation of the actin cytoskeleton by Rho GTPases, the role of PKA in cytoskeleton remodeling and integrin mediated cell adhesion. Signaling pathways including G-protein signaling - RhoA regulation pathway and $G$ protein- $\alpha 12$ signaling pathways were also identified in the proteome pathway enrichment analysis. The generated pathway maps correlate with the proteome data that reveal a large proportion (12\%) of LCV proteins are involved in cytoskeletal formation of the LCV.

Bioinformatic analysis of metabolic networks in the WT strain LCV proteome revealed the phosphatidylinositol-4,5-diphosphate pathway and phosphatidylinositol3,4,5-triphosphate pathway as two of the top five (Fig. 11). These data reflect the aforementioned results of a wide array of phosphatidylinositol-dependent proteins in the WT strain and the ankB mutant LCV proteomes. This is also consistent with findings that have shown the LCV is decorated with phospatidylinositol-4 phosphate (PI(4)P) to which multiple effector proteins (SidC, SidM, SdcA, RidL) bind on the LCV membrane [74, 80]. Carbohydrate metabolism (glycolysis, gluconeogenesis and glucose transport) was the third most abundant metabolomic network identified; while valine, tryptophan and methionine synthesis and transport were identified in the top eight metabolic pathways represented in the LCV proteome (Fig. 11). Performing the MetaCore analysis on the 
ankB mutant strain LCV proteome gave similar results to the WT strain LCV, such as the phosphatidylinositol-4,5-diphosphate pathway and phosphatidylinositol-3,4,5triphosphate pathway.

When proteins found only on the WT strain LCV but not the ankB mutant LCV were analyzed through MetaCore, many of the pathways were consistent with the total WT strain LCV proteome, such as phosphatidylinositol-4,5-diphosphate pathway. The finding that the $a n k B$ mutant proteome contained $80 \%$ of the proteins present in the WT strain LCV proteome is consistent with the MetaCore analysis of very similar pathways in both of the proteomes. Interestingly, Ubiquitin-proteasomal proteolysis was identified as the second most abundant process network involved in WT strain LCV specific proteins despite the findings that the $a n k B$ mutant LCV proteome contains a majority of the proteins found in the WT strain LCV proteome. These results are consistent with the requirement of polyubiquitination of the WT strain LCV for intracellular replication. Another explanation could be due to the WT strain LCV containing 350 less proteins than the ankB mutant strain, therefore the increase in lysine ${ }^{48}$-linked polyubiquitinated proteins, proteasomal subunit proteins or the SCF1 E3 ubiquitin ligase components recruited to the WT LCV would be more significant due to the decreased number of total proteins identified in the WT LCV proteome. 


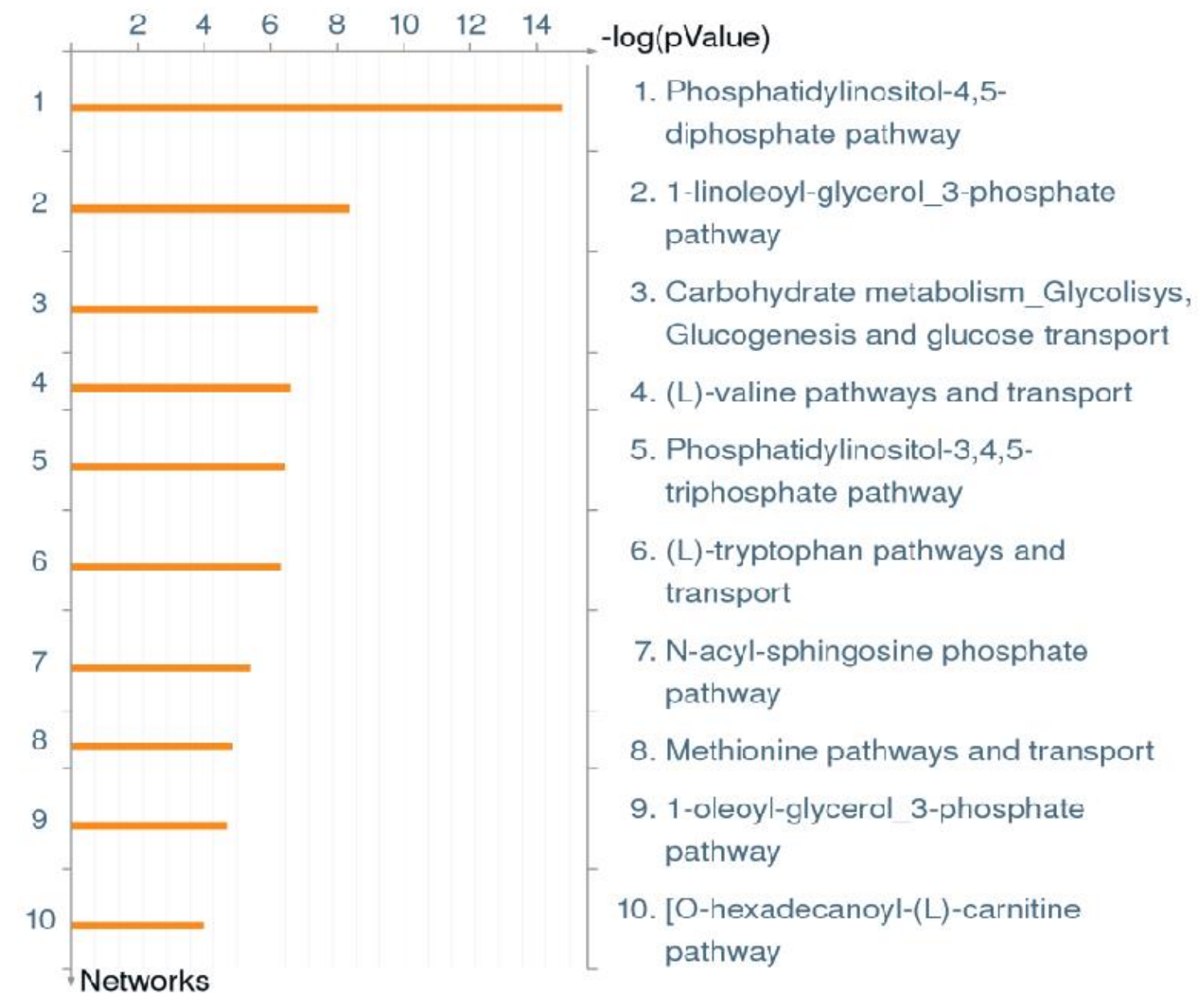

Fig. 11. MetaCore enrichment analysis of metabolic networks in the WT Strain

LCV proteome. The complete WT strain LCV proteome was analyzed through MetaCore software, using the enrichment analysis function, to identify the most significant metabolic networks. 


\section{The ubiquitinated proteome of the LCV}

To identify ubiquitinated proteins on the WT and ankB mutant LCV, the solubilzed LCV proteins were immunoprecipitated with anti-ubiquitin antibodies and identified by LC/MS. We identified 24 ubiquitinated proteins on the WT strain LCV such as Annexin A2, Plasminogen activator inhibitor, Rab5 GDP/GTP exchange factor and Transitional endoplasmic reticulum ATPase (p97) (Table 2). The polyubiquitinated proteins identified on the $\mathrm{LCV}$ were $\mathrm{K}^{6}, \mathrm{~K}^{11}, \mathrm{~K}^{27}$ and $\mathrm{K}^{48}$-linked as evident from the findings that ubiquitin was ubiquitinated on the 4 lysine residues $\mathrm{K}^{6}, \mathrm{~K}^{11}, \mathrm{~K}^{27}$ and $\mathrm{K}^{48}$. Interestingly, 6 (25\%) of the ubiquitinated proteins on the WT strain LCV play a role in the immune response such as interleukin-1 receptor-associated kinase 1 and Interferon regulatory factor 7 , which play a key role in the signaling and regulation of the immune system against pathogens. Furthermore, we identified 2 amino acid transporters (SLC3A2 and SLC1A4) and a sodium bicarbonate transporter (SLC4A7) to be ubiquitinated on the WT strain LCV. We analyzed these proteins through the Mammalian Ubiquitination Site Database [271] which contains a comprehensive list of all ubiquitination sites on mammalian proteins. The search revealed that 17 of the identified ubiquitinated proteins have been shown to be ubiquitinated on the same lysine residues as identified in our LC/MS analyses. However, three of the ubiquitinated proteins identified (Plasminogen activator inhibitor 2, Mast cell-expressed membrane protein 1, Isoform 13 of Sodium bicarbonate cotransporter 3) were not in the database, while four (Vimentin, Target of Myb protein 1, Receptor-type tyrosine-protein phosphatase C, Transient receptor potential cation channel subfamily $\mathrm{V}$ member 2 ) were in the database but have been previously shown to be ubiquitinated on other lysine residues than the ones we identified 
in our analysis. The ankB mutant LCV contained 29 ubiquitinated proteins primarily involved in signaling (Integrin beta-1, beta-2 and alpha-5, Rho GDP-dissociation inhibitor 2) and ubiquitination (Cullin-5, the E3 ubiquitin ligase LAPTM5 and the E2 Ubiquitin-conjugating enzyme E2N) (Table 3). The ankB mutant LCV had multiple ubiquitinated transporters, including the monocarboxylate transporter (SLC16A3), the glucose transporter (SLC2A3), and neutral amino acid transporters (SLC3A2, SLC1A5) as well as 2 ATPases (ATP2B4 and ATP13A3) (Table 3).

While the WT strain and ankB mutant strain LCV had some common ubiquitinated proteins (p97, SLC3A2, tubulin), there was a significant difference in the ubiquitinated proteome among the two strains, as can be seen by the superscript alpha designation in the WT strain ubiquitinated proteome table (Table 3). The major differences in the ubiquitinated host proteins between the two strains could be attributed to the proteasomal degradation of $\mathrm{K}^{48}$-linked polyubiquitinated proteins on the WT strain $\mathrm{LCV}$, and the dynamic changing nature of LCV. The ankB mutant LCV had multiple ubiquitinated proteins involved in intracellular trafficking (Rab1A, Rab14) and two GTPases (Rac1, RhoG), while the WT strain only contained one ubiquitinated protein involved in intracellular trafficking (Rab5 GDP/GTP exchange factor) (Table 3-4). Furthermore, the ubiquitinated proteins on the WT strain LCV that were excluded from the ankB mutant strain LCV can be utilized to determine the AnkB substrates, that are required for intracellular replication. 
Table 3.

Ubiquitinated proteome of the WT strain LCV

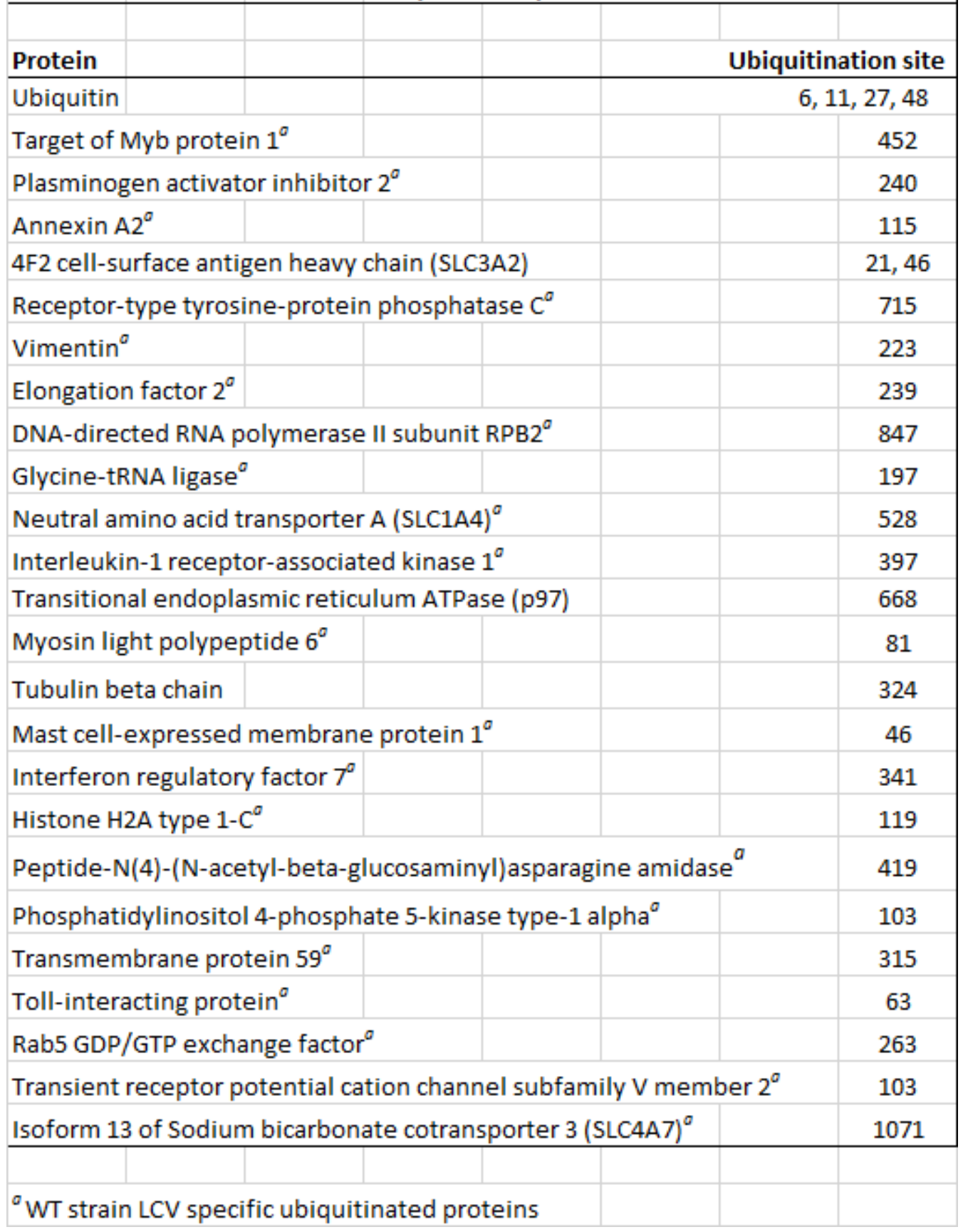


Table 4.

Ubiquitinated proteome of the ankB mutant LCV

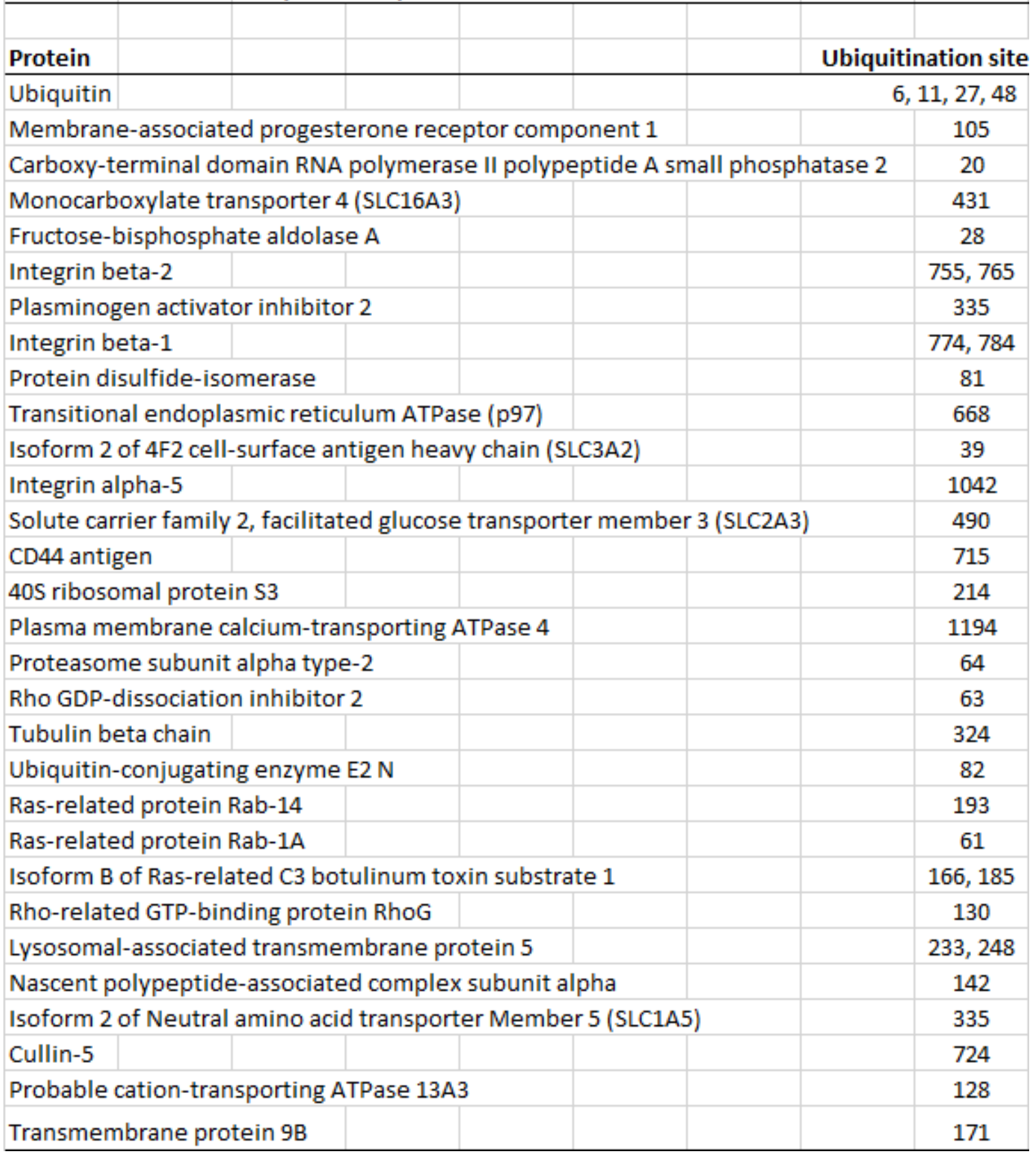




\section{Discussion Section 2}

While the LCV proteome has been characterized from the mouse macrophage cell line RAW264.7 and D. discoedium $[73,74]$, there has not been a complete LCV proteome analysis from human macrophages. While RAW264.7 mouse macrophages support intracellular replication, they originate from the BALB/C mouse, which are non-permissive to L. pneumophila infection [76, 272]. Therefore, generation of the LCV proteome from human macrophages is a valuable resource for studies focusing on LCV biogenesis within human cells. Due to the requirement of $\mathrm{K}^{48}$-linked polyubiquitinated proteins on the $\mathrm{LCV}$, determining the ubiquitinated proteome of the LCV will show host proteins modified during infection that could be crucial for intracellular replication. Utilizing the ankB mutant strain as a control for generating the proteome is valid due to the similarity in LCV biogenesis and trafficking to the WT strain. In addition, utilizing the $a n k B$ mutant strain allowed the identification of WT strain-specific ubiquitinated proteins, some of which could be ankB substrates that are required for intracellular replication.

The WT strain LCV proteome contained a unique assortment of E2 ubiquitinconjugation enzymes and E3 ubiquitin ligases. Out of the 4 E2 ubiquitin-conjugation enzymes (UBE2K, UBE2N, UBE2L3, UBEV1) only UBE2K and UBE2L3 synthesize K ${ }^{48}$ linked polyubiquitinated chains [268]. The WT strain LCV proteome confirmed previous results of the SKP1 component of the SCF1 E3 ubiquitin ligase complex and VCP (p97) localized to the LCV [237, 241]. Interestingly, SKP1 and VCP are needed for intracellular replication, due to SKP1 ubiquitinating AnkB substrates and VCP removing the $\mathrm{K}^{48}$-linked polyubiquitinated proteins from the LCV for proteasomal degradation [237, 238]. Therefore, UBE2K and UBE2L3 could be the missing piece of LCV polyubiquitination, and function to 
polymerize the $\mathrm{K}^{48}$-linked polyubiquitination of the AnkB substrates. Interestingly, we identified multiple ubiquitin specific peptidases on the LCV which function in a regulatory role to prevent proteasomal degradation of the ubiquitinated protein [204]. Ubiquitin peptidases remove conjugated ubiquitin from linkages other than $\mathrm{K}^{48}$, which complicates the identification of LCV associated ubiquitinated proteins. These peptidases could also alter the state of LCV ubiquitination, thereby influencing protein turnover in the infected cell. The function of the E3 ubiquitin ligases on the LCV could prove valuable to determine if they are modifying bacterial proteins or if they are recruited to the LCV by effector proteins in a manner to direct ubiquitination of specific target proteins. The E3 ubiquitin ligase Huwe1 found in the WT strain LCV proteome regulates apoptosis through ubiquitination of Mcl-1 [267], while the E3 ubiquitin ligase Birc6 found on the WT strain and ankB mutant strain LCV ubiquitinates apoptotic proteins in a proteasomal dependent manner [266]. Other antiapoptotic proteins on the LCV such as defender against cell death could play an important role in preventing host cell apoptosis and therefore limiting bacterial replication. The proapoptotic proteins on the LCV, such as apoptosis-inducing factor, show a unique balance between pro and anti-apoptotic events during L. pneumophila infection. This balance is of importance due to L. pneumophila activating caspase 3, which does not lead to apoptosis, as well as determining why permissive macrophages are resistant to apoptotic stimuli during infection $[62,63,67,68]$.

Due to the essential recruitment of polyubiquitinated proteins to the LCV in L. pneumophila strain AA100/130b and the Paris strain [238, 239], we sought to determine the identity of these ubiquitinated proteins to decipher which host proteins were manipulated during infection of human macrophages. Six of the 24 ubiquitinated proteins found on the 
WT strain LCV are involved in the immune response. These ubiquitinated immune response proteins include interferon regulatory factor 7 (IRF7) and interleukin-1 receptor-associated kinase 1 (IRAK1). IRF7 is a key transcriptional regulator of type I interferon (IFN)dependent immune responses, which is critical for clearance of many DNA and RNA viruses [273]. Interestingly, the E3 ubiquitin ligase Trim21 ubiquitinates IRF7 in a proteasomal degradation manner [274]. Furthermore, IRF7 ubiquitination could also contribute to the inhibition of apoptosis during L. pneumophila infection. IRAK1 plays a role in mediating pro-apoptotic signaling in response to bacterial LPS [275]. Therefore, similar to IRF7, the ubiquitination of IRAK1 could play a significant role in intracellular immune response during infection [273]. The ankB mutant strain LCV ubiquitinated proteome contained Rab1A and Rab14. While Rab1A plays a crucial role in LCV remodeling and trafficking [78], this ubiquitination could explain an additional regulatory mechanism for LCV biogenesis and endo-lysosomal evasion. Additionally, the ankB mutant strain ubiquitinated proteome contained multiple proteins involved in ubiquitination and amino acid transport, such as the ubiquitin-conjugating enzyme E2N, cullin5, SLC1A5 and SLC3A2. The WT strain ubiquitinated proteome also contained the amino acid transporter SLC3A2 as well as the amino acid transporter SLC1A4. The ubiquitination of these amino acid transporters could alter their subcellular localization to enable their localization to the LCV. This could explain how intra-vacuolar L. pneumophila is able to transport amino acids across the LCV lumen to provide the carbon and energy essential for intracellular replication. The ubiquitinated proteome of the WT and ankB mutant strain LCV each contained $\mathrm{K}^{6}, \mathrm{~K}^{11}, \mathrm{~K}^{27}$ and $\mathrm{K}^{48}$-linked polyubiquitinated proteins. Further studies are needed to determine the chain linkages of the ubiquitinated proteins as well as characterizing the importance of the non- 
$\mathrm{K}^{48}$-linked polyubiquitinated proteins. While it is known the $\mathrm{K}^{48}$-linked polyubiquitinated proteins are essential for intracellular replication [86], the other ubiquitinated proteins could prove valuable to decipher vacuole remodeling and biogenesis.

L. pneumophila must acquire nutrients primarily in the form of amino acids from the host during infection [86, 262]. It is not known how amino acids are transported into the LCV lumen, however it is known that the eukaryotic amino acid transporter SLC1A5 is required for intracellular replication within human macrophages [85]. In the WT strain and the ankB mutant strain LCV proteomes we identified five amino acid transporters (SLC1A4, SLC1A5, SLC3A2, SLC7A5, SLC38A2). While SLC1A5 is required for L. pneumophila infection of human macrophages [85], there could be multiple amino acid transporters on the LCV that function together to bring a variety of amino acids into the LCV. The more important question is what directs these amino acid transporters to the LCV and how they anchor into the LCV membrane. A transmembrane L. pneumophila effector could bind these transporters and stabilize them on the LCV membrane allowing import of amino acids, or the transporters could fuse with the LCV by recruitment of ER vesicles. We currently favor the second possibility, since the SLC transporters are transmembrane proteins. Due to the wide array of protozoan host L. pneumophila infects, having the ability to localize multiple amino acid transporters to the LCV would be a beneficial solution to nutrient acquisition in various host.

The LCV proteome also contained two monocarboxylate transporters SLC16A1 and SLC16A3 that have the ability to transport pyruvate across the LCV membrane [276]. While amino acids are the main source of nutrients, the ankB mutant is restored for intracellular replication in human macrophages and amoeba when pyruvate is added to the culture 
medium [86]. Therefore, incorporation of these monocarboxylate transporters into the LCV membrane could provide a dual strategy for L. pneumophila acquisition of sources other than amino acids, such as pyruvate. L. pneumophila has been shown to use glucose as an intracellular energy source [92], the glucose transporters identified in the LCV proteomes (SLC2A1, SLC2A3, SLC2A13) could allow another, yet minor, source of carbon and energy to support intracellular replication. With our knowledge of L. pneumophila nutrient acquisition not well understood, this proteome analysis in human macrophages will be a tremendous resource to enable studies into more specific transporters not previously known to be localized to the LCV. While SLC1A5 is required for infection it is more likely that combinations of amino acid, monocarboxylate and glucose transporters are recruited to the LCV to provide intra-vacuolar L. pneumophila with a diverse portfolio of sources of carbon and energy. 


\section{Results Section 3. Lysine ${ }^{11}$-linked polyubiquitination of the AnkB effector of Legionella pneumophila}

\section{Ubiquitination of the L. pneumophila translocated effector AnkB}

It has been shown that eukaryotic F-box proteins undergo autoubiquitination through the direct interaction with the SCF1 E3 ubiquitin ligase complex [221, 226]. However, to our knowledge ubiquitination of F-box proteins independent of their F-box domain has not been investigated. To further investigate the function of F-box proteins we choose to further characterize the eukaryotic F-box L. pneumophila effector protein AnkB.

To determine whether AnkB undergoes ubiquitination within eukaryotic cells, we immunoprecipitated ectopically expressed Flag-tagged AnkB and analyzed by Western Blot. In addition to the native AnkB band at $\sim 23 \mathrm{kDa}$ there were two distinct bands at $\sim 37 \mathrm{kDa}$ and $\sim 75 \mathrm{kDa}$, as well as a smear of high molecular weight proteins recognized by anti-Flag antibodies (Fig. 12). No bands were detected in the lysate of cells transfected with the vector alone, which suggested that the distinct bands at $\sim 37 \mathrm{kDa}$ and $\sim 75 \mathrm{kDa}$ may correspond to the potential addition of two and six ubiquitin monomers to AnkB since 1 ubiquitin monomer is $8.5 \mathrm{kDa}$ (Fig. 12). Re-probing that membrane with antiubiquitin antibodies on AnkB showed the presence of higher molecular mass species that were ubiquitinated (Fig. 1). To ensure that ubiquitination of AnkB was not only limited to the L. pneumophila strain AA100/130b we also analyzed AnkB from the L. pneumophila Paris strain [239], which lacks the C-terminal CaaX motif [135]. We performed immunoprecipitation of Flag-tagged AnkB Paris and probed the membrane with anti-Flag antibodies. Similar to the AA100/130b-derived AnkB, the Paris strain AnkB showed 
distinct bands and a high molecular weight smear that suggested the ubiquitination of AnkB (Fig. 12).

AnkB is a bona fide F-box protein [238, 239] and its F-box domain interacts directly with the SKP1 component of the host SCF1 E3 ubiquitin ligase complex on the LCV [238, 239]. To determine if the F-box domain of AnkB was required for its ubiquitination, we transfected HEK293T cells with an AnkB variant lacking the F-box domain and immunoprecipitated the protein. Western blot analysis showed the deletion of the F-box domain of AnkB did not have an impact on its ubiquitination (Fig. 13). To ensure that deletion of the F-box domain did not have an impact on the structure of AnkB, we utilized an AnkB substitution mutant $\left(\mathrm{AnkB}^{9} \mathrm{~L}^{10} \mathrm{P} / \mathrm{AA}\right)$ within the F-box domain that does not bind to SKP1 [238]. Following immunoprecipitation of $\mathrm{AnkB}^{9} \mathrm{~L}^{10} \mathrm{P} / \mathrm{AA}$, the western blot showed that $\left(\mathrm{AnkB}^{9} \mathrm{~L}^{10} \mathrm{P} / \mathrm{AA}\right)$ was ubiquitinated similar to the native AnkB (Fig. 13). Taken together these results highly suggest that AnkB is ubiquitinated and its ubiquitination is independent of the interaction of its F-box domain with the host SCF1 E3 ubiquitin ligase complex, which indicates that AnkB is modified by a host ubiquitin ligase. 
A

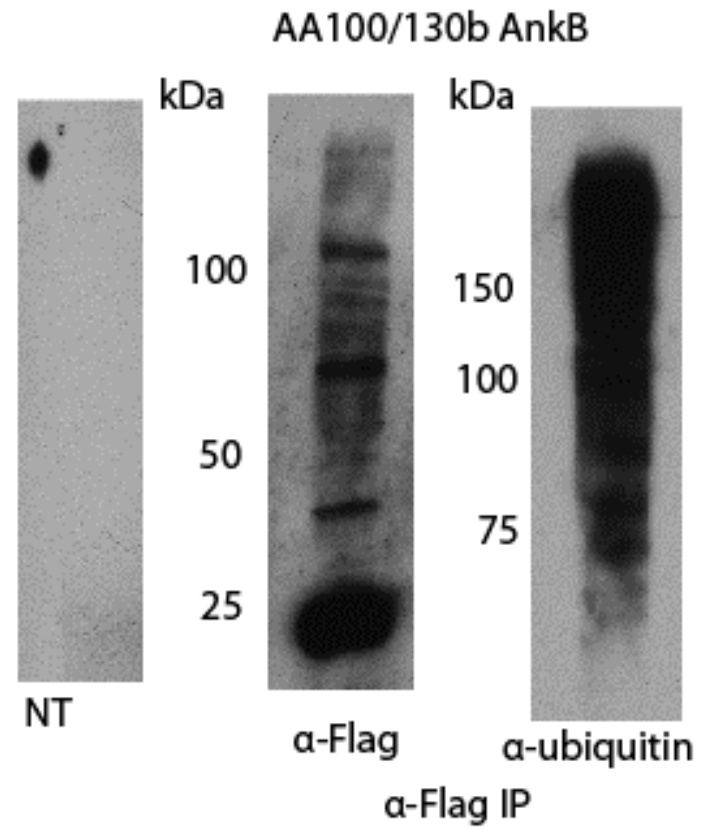

B

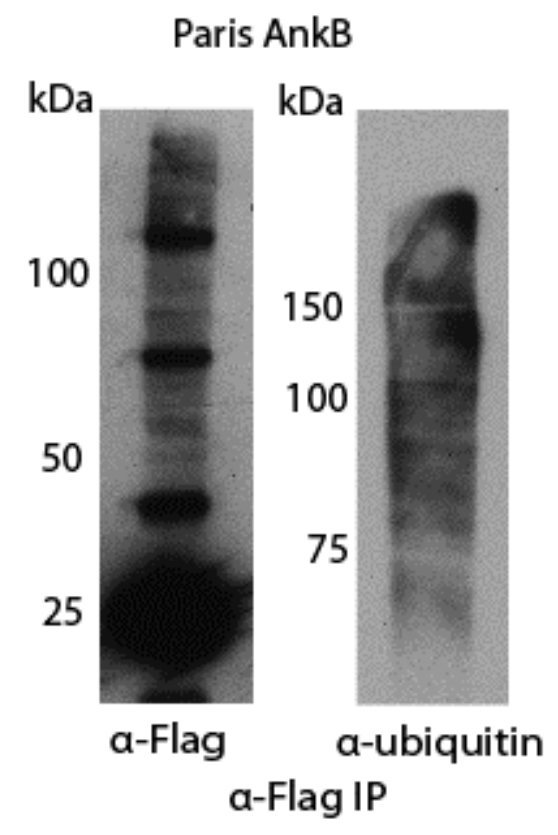

Fig. 12. Ubiquitination of ectopically expressed L. pneumophila strain AA100/130b and the Paris strain AnkB. HEK293T cells were transfected with 3X-Flag tagged AnkB from L. pneumophila strain AA100/130b or the Paris strain. Cell lysates were purified with anti-Flag agarose and analyzed by immunoblotting with anti-Flag and anti-ubiquitin antibodies. 


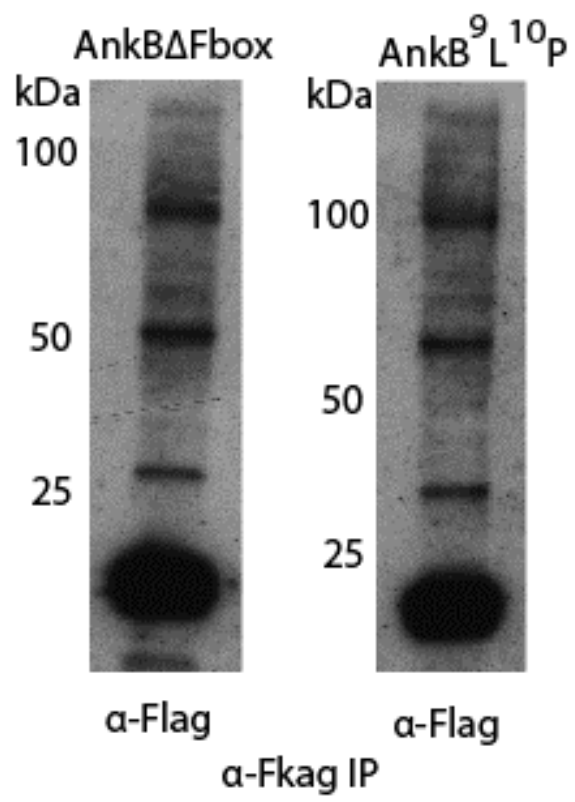

Fig. 13. Ubiquitination of AnkB variants lacking the F-box domain.

HEK293T cells were transfected with the AnkB variants $3 X F l a g$-tagged $A n k B{ }^{9} \mathrm{~L}^{10} \mathrm{P}$ or 3XFlag-tagged AnkB $\Delta$ Fbox. Cell lysates were purified with anti-Flag agarose and analyzed by immunoblotting with anti-Flag antibodies

\section{Interaction of AnkB with the E3 ubiquitin ligase Trim21}

The ubiquitination of the AnkB variant lacking the F-box domain, as well as the AnkB ${ }^{9} \mathrm{~L}^{10} \mathrm{P} / \mathrm{AA}$ substitution variant allowed us to exclude a role for the SCF1 E3 ubiquitin ligase in AnkB ubiquitination. We came to this conclusion since AnkB is unable to bind the SKP1 component in either of these AnkB flag-tag variants $\left(\mathrm{AnkB}^{9} \mathrm{~L}^{10} \mathrm{P}\right.$ and $A n k B \Delta F b o x)$, therefore it is highly unlikely that the ubiquitination of AnkB is dependent upon the SCF1 E3 ubiquitin ligase complex. In addition the targets of AnkB 
can not be ubiquitinated by AnkB in the absence of the F-box domain of AnkB, therefore the ubiquitination of the $\mathrm{AnkB}$ variants $\left(\mathrm{AnkB}^{9} \mathrm{~L}^{10} \mathrm{P}\right.$ and $\left.\mathrm{AnkB} \Delta \mathrm{Fbox}\right)$ highly suggest AnkB, and not its interacting substrates, are ubiquitinated through an E3 ubiquitin ligase complex other than the SCF1 complex. Therefore, we sought to determine the E3 ubiquitin ligase that interacts with AnkB. We immunoprecipitated Flag-tagged AnkB from HEK293T cells and performed MS analysis on the proteins that coimmunoprecipitated with AnkB. Our mass spectrometry data showed that the RINGfinger protein Ro52 (Trim21) co-purified with AnkB. To confirm AnkB directly interacts with Trim21, we co-transfected HEK293T cells with Flag-tagged AnkB and HA-tagged Trim21. Following cell lysis, Flag-tagged AnkB was co-immunoprecipitated with antiFlag antibody and the immunoprecipitate was immunoblotted with anti-HA tag antibody. A $\sim 52 \mathrm{kDa}$ band, the correct molecular mass for Trim21, co-immunoprecipitated with AnkB (Fig. 14A). When the co-immunoprecipitation was reversed through immunoprecipitation with anti-HA antibody and the immunoprecipitate was immunoblotted with anti-Flag antibodies, the correct molecular mass band corresponding to AnkB was detected (Fig. 14B). To determine if the Trim21-AnkB interaction was specific for AnkB, we utilized the L. pneumophila translocated effector protein AnkH as a control in the co-immunoprecipitation. The Flag-tagged AnkH was coimmunoprecipitated with anti-Flag antibodies and the resulting immunoprecipitate was immunoblotted with anti-HA antibodies. Unlike AnkB, Trim21 did not coimmunoprecipitate with AnkH (Fig.14A). This was confirmed by reversing the coimmunoprecipitation and Western Blot, which also showed lack of interaction of Trim21 with the AnkH control (Fig. 14B). Taken together, these results indicate AnkB 
specifically interacts with the E3 ubiquitin ligase Trim21. This is the first example of an interaction of a bacterial effector with the host Trim21 ubiquitin ligase.

A

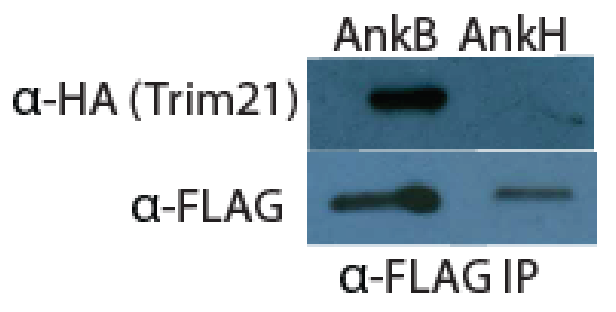

B

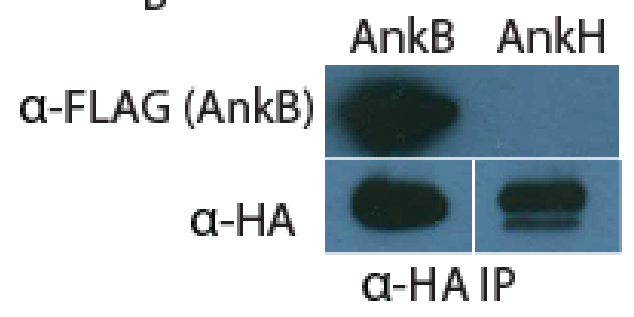

Fig. 14.AnkB directly interacts with the E3 ubiquitin ligase Trim21. (A) Flag-tagged AnkB or Flag-tagged AnkH were co-transfected with HA-tagged Trim21 in HEK293T cells. Flag-tagged AnkB or Flag-tagged AnkH were co-immunoprecipitated with antiFlag agarose and the resulting supernatants were immunoblotted with anti-HA antibodies and anti-Flag antibodies. (B) HA-tagged Trim21 was co-immunoprecipitated using antiHA agarose and the resulting supernatants were analyzed by immunoblotting with antiFlag antibodies and anti-HA antibodies.

\section{Ubiquitinated AnkB is not degraded by the proteasome}

When proteins are polyubiquitinated through $\mathrm{K}^{48}$-linked polymerization of ubiquitin are degraded by the proteasome [202]. To determine whether ubiquitination of AnkB led to its proteasomal degradation we utilized the proteasomal inhibitor MG132 
and determined the stability of AnkB in the presence or absence of the protein synthesis inhibitor cycloheximide. To ensure the MG132 proteasome inhibitor blocked proteasomal degradation, treated or untreated cells were lysed and the resulting western blot membrane of total cell lysate was probed with anti-ubiquitin and re-probed with antiactin antibodies. As expected, cells treated with MG132 had a large increase in the amount of ubiquitinated proteins compared to untreated cells (Fig. 15A). When AnkB was co-immunoprecipitated from cells left untreated or treated with MG132 there was not a difference in the amount of purified ubiquitinated AnkB (Fig. 15B). Our data showed that upon proteasomal inhibition, ubiquitinated AnkB was stable over the three hour experiment (Fig. 15C). Therefore, ubiquitination of AnkB does not result in its proteasomal degradation or shortening of its half-life (Fig. 15C). 
A

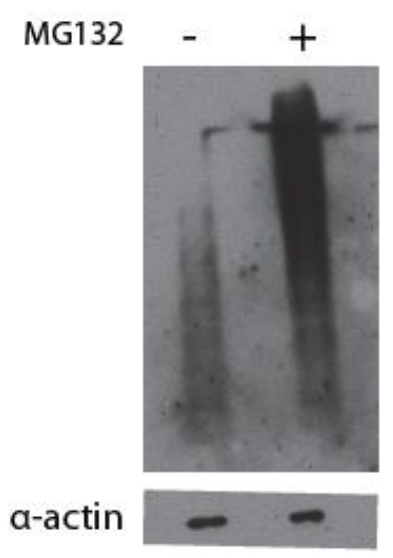

B

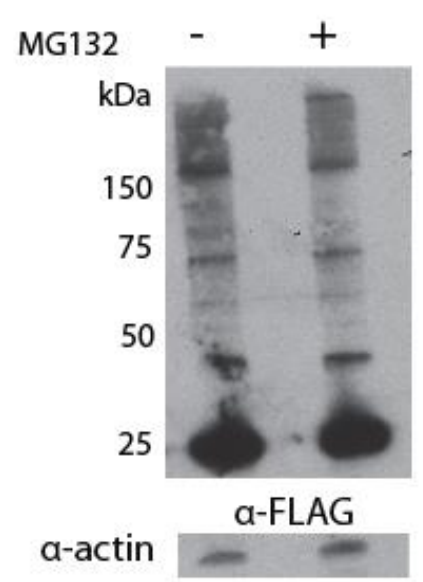

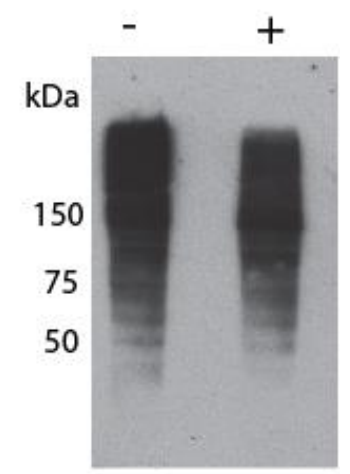

a-ubiquitin

a-FLAG IP
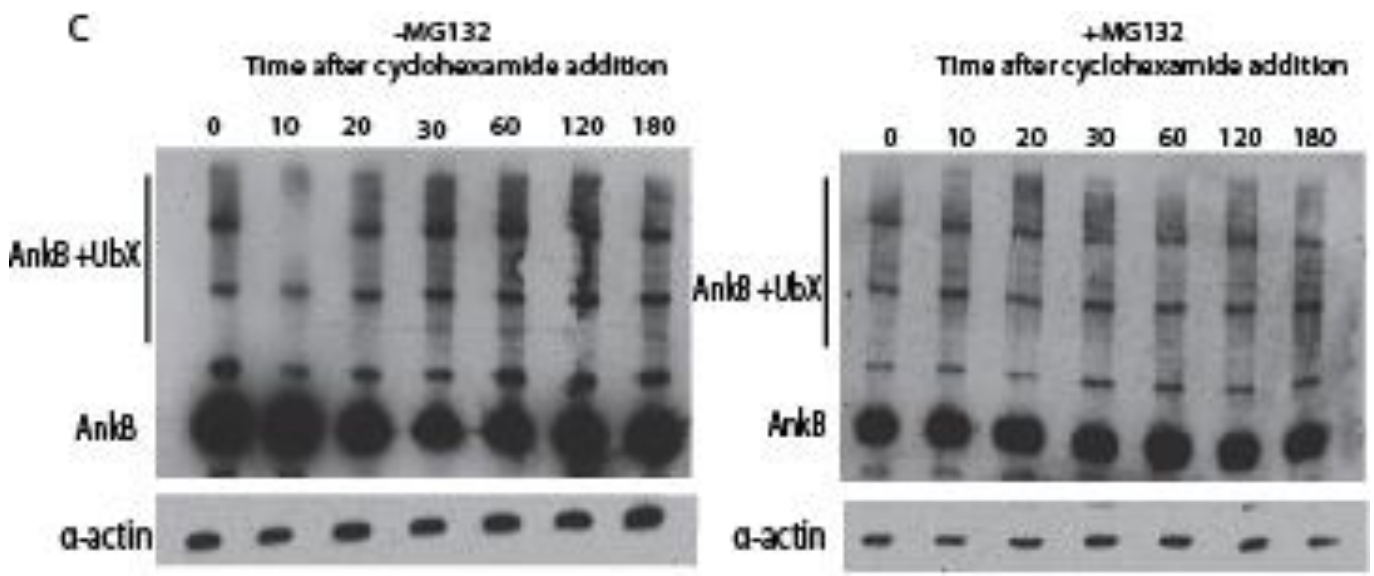

Fig.15. Ubiquitinated AnkB is not degraded by the proteasome. (A) HEK293T cells were untreated or treated with 20uM MG132 for 3 hours. Cells were lysed and the resulting immunoblot was probed with anti-ubiquitin and anti-actin antibodies. (B) HEK293T cells transfected with 3X-Flag tagged AnkB were treated with 20uM MG132 for 3 hours or left untreated. An equivalent number of cells were lysed, and Flag-tagged AnkB was immunoprecipitated using anti-Flag agarose, and subjected to immunoblotting with anti-Flag and anti-ubiquitin antibodies. (C) HEK293T cells transfected with Flag tagged AnkB were treated with the protein synthesis inhibitor cycloheximide. To inhibit 
proteasomal degradation, a subset of the cells were treated with 20uM MG132 two hours prior to cycloheximide treatment. At the indicated time points cells were lysed and equivalent amounts of protein were subjected to immunoblotting with anti-Flag and antiactin antibodies.

\section{Polyubiquitin linkages of polyubiquitinated AnkB}

Ubiquitin contains 7 lysine residues, all of which can be utilized to polymerize ubiquitin on the substrate protein [202]. To determine if AnkB was polyubiquitinated through $\mathrm{K}^{48}$ and $\mathrm{K}^{63}$-linked polyubiquitination, we utilized antibodies specific for $\mathrm{K}^{48}$-and $\mathrm{K}^{63}$-linked polyubiquitinated proteins. These are the most studied forms of polyubiquitination, therefore there are commercial antibodies available. The immunoprecipitated AnkB did not bind antibodies specific for $\mathrm{K}^{48}$ and $\mathrm{K}^{63}$-linked polyubiquitinated proteins compared to total cell lysate control (Fig. 16). When the membrane was stripped and re-probed with anti-Flag antibodies, ubiquitinated AnkB was detectable (Fig. 16). Taken together, these results highly suggest that AnkB is polyubiquitinated through lysine linkages other than $\mathrm{K}^{48}$ or $\mathrm{K}^{63}$. If $\mathrm{AnkB}$ was polyubiquitinated through $\mathrm{K}^{48}$ or $\mathrm{K}^{63}$ we would expect to see a similar high molecular weight smear that was observed with the anti-ubiquitin antibodies (15B).

Since AnkB is most likely not modified through $\mathrm{K}^{48}$ or $\mathrm{K}^{63}$-linked polyubiquitination we sought to determine the chain linkage of the ubiquitin polymers of ubiquitinated AnkB by mass spectrometry. Depending on the lysine residue within 
ubiquitin that contains the ubiquitin-ubiquitin linkage, a characteristic mass shift increase of $114 \mathrm{Da}$ (di-glycine) is observed by mass spectrometry [242]. The Flag-tagged AnkB was ectopically expressed in HEK293T cells and immunoprecipitated using anti-Flag antibody. Immunoprecipitated proteins were resolved by SDS-PAGE and visualized by Coomassie blue staining (Fig. 17). Of the several bands corresponding to AnkB with a different number of ubiquitin moieties, the most prominent band at $\sim 75 \mathrm{kDa}$ (AnkB +6 ubiquitin moieties) was analyzed by mass spectrometry to determine the modified lysine residues within AnkB and ubiquitin. Mass spectrometry analysis and the resulting spectra detected ubiquitination of AnkB on $\mathrm{K}^{67}$ in the peptide (63-75), that was generated following trypsin cleavage of the $\sim 75 \mathrm{kDa}$ band (Fig. 18A). Greater than $90 \%$ of the $\mathrm{b}$ and y ions with $+1,+2$ and +3 charges were detected, which provided clear evidence for the ubiquitination of AnkB on $\mathrm{K}^{67}$ (Fig. 18A and B). When analyzing other bands from the Coomassie stained gel by mass spectrometry we did not observe ubiquitination of AnkB, however, many of the lysine residues within AnkB were not even observed following protein digestion. Within the same $\sim 75 \mathrm{kDa}$ band, ubiquitin was ubiquitinated on $\mathrm{K}^{11}$ in the peptide (7-27) (Fig. 19). The $b$ ions with $a+1$ charge and the y ions with $a+2$ charge surrounding the modified lysine residue of ubiquitin are shown and the calculation provided direct evidence for ubiquitination of ubiquitin on $\mathrm{K}^{11}$ (Fig. 19A and B). Although phosphorylation of proteins has been shown to be a signal for protein ubiquitination [277], we did not detect phosphorylated AnkB in the MS analysis (data not shown). If the phosphorylated amino acid of AnkB was detected we could possibly substitute the phosphorylated residue to inhibit the ubiquitination of AnkB. To inhibit host cell ubiquitination we generated a substitution mutant of the ubiquitinated $\mathrm{K}$ residue 
in $\mathrm{AnkB}\left(\mathrm{AnkBK}{ }^{67} \mathrm{R}\right)$. However, following immunoprecipitation the resulting immunoblot analysis showed AnkB ubiquitination was not inhibited with this substitution (Fig. 20). As seen with many eukaryotic proteins and the SopB effector in Salmonella, ubiquitination can occur on multiple K residues within a protein [234, 278]. Taken together, AnkB is ubiquitinated on $\mathrm{K}^{67}$ through $\mathrm{K}^{11}$-linked polyubiquitination, and the functional result of the $\mathrm{K}^{11}$-linked polyubiquitination remains to be determined.

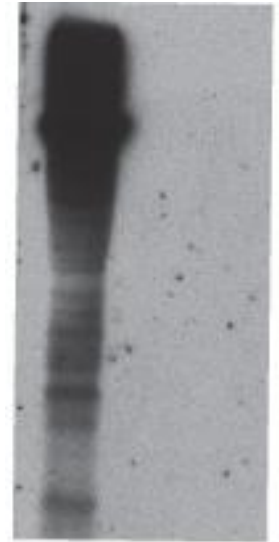

Input AnkB AnkB a-K48 a-FLAG
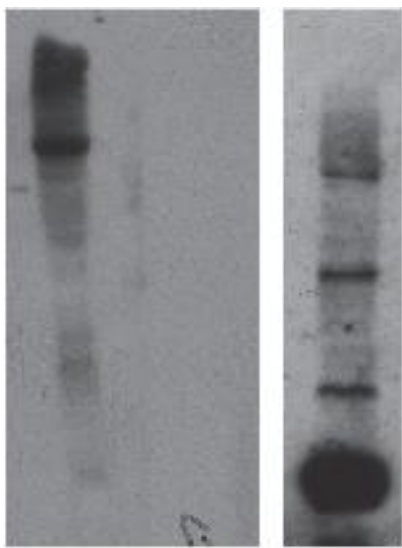

Input AnkB AnkB a-K63 a-FLAG

Fig. 16. AnkB is not polyubiquitinated through $K^{48}$ or $K^{63}$ linkages.

Immunoprecipitated Flag-tagged AnkB was analyzed by immunoblotting with antibodies specific for $\mathrm{K}^{48}$-linked polyubiquitinated proteins or antibodies specific for $\mathrm{K}^{63}$-linked polyubiquitinated proteins. Cell lysate was used as a control and anti-Flag antibodies were used to show ubiquitinated Flag-tagged AnkB. 


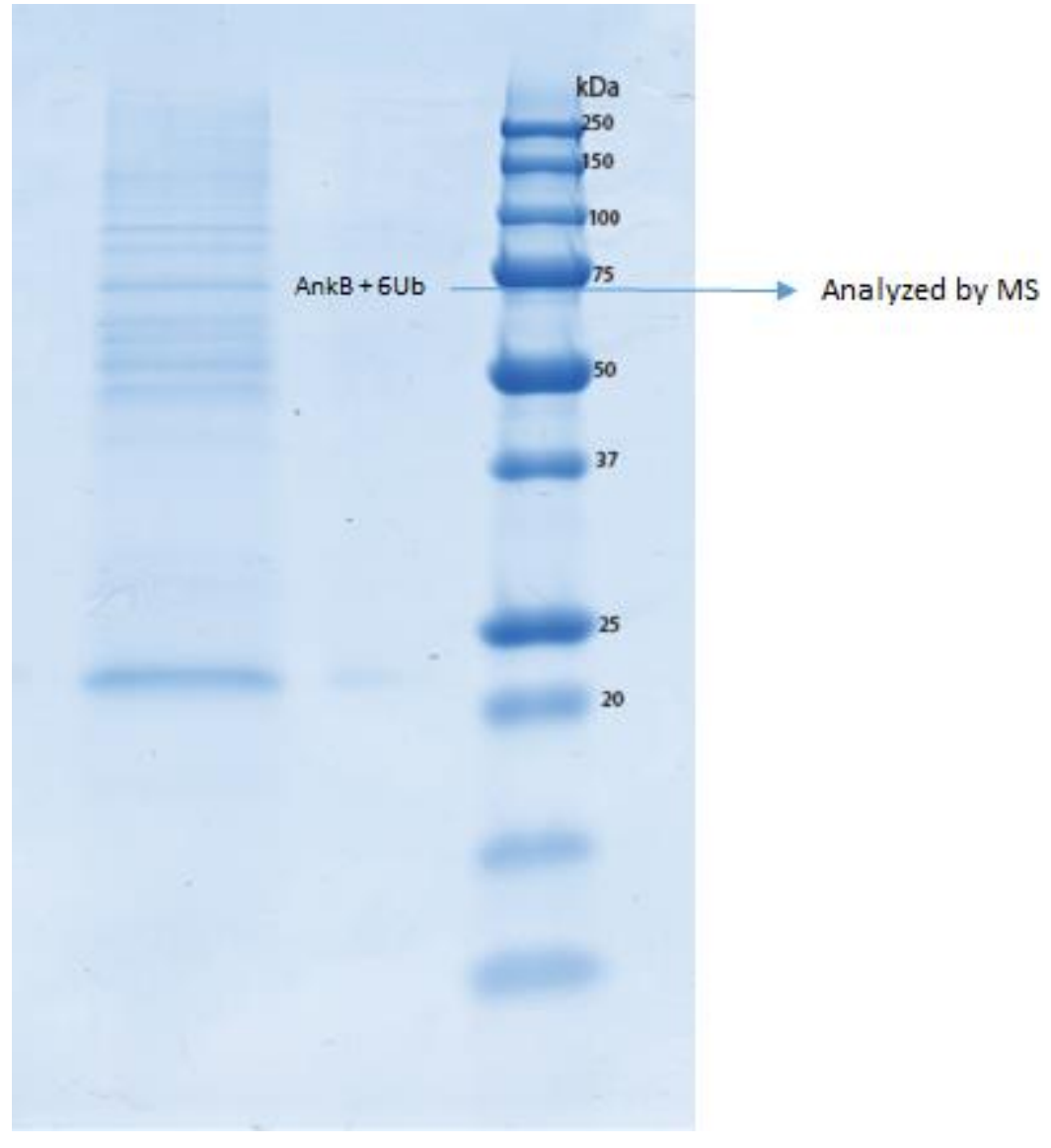

Fig. 17. Commassie stain of immunoprecipitated AnkB.

Flag-tagged AnkB was immunoprecipitated from transfected HEK293T cells and analyzed by SDS-PAGE and Commassie blue staining. 

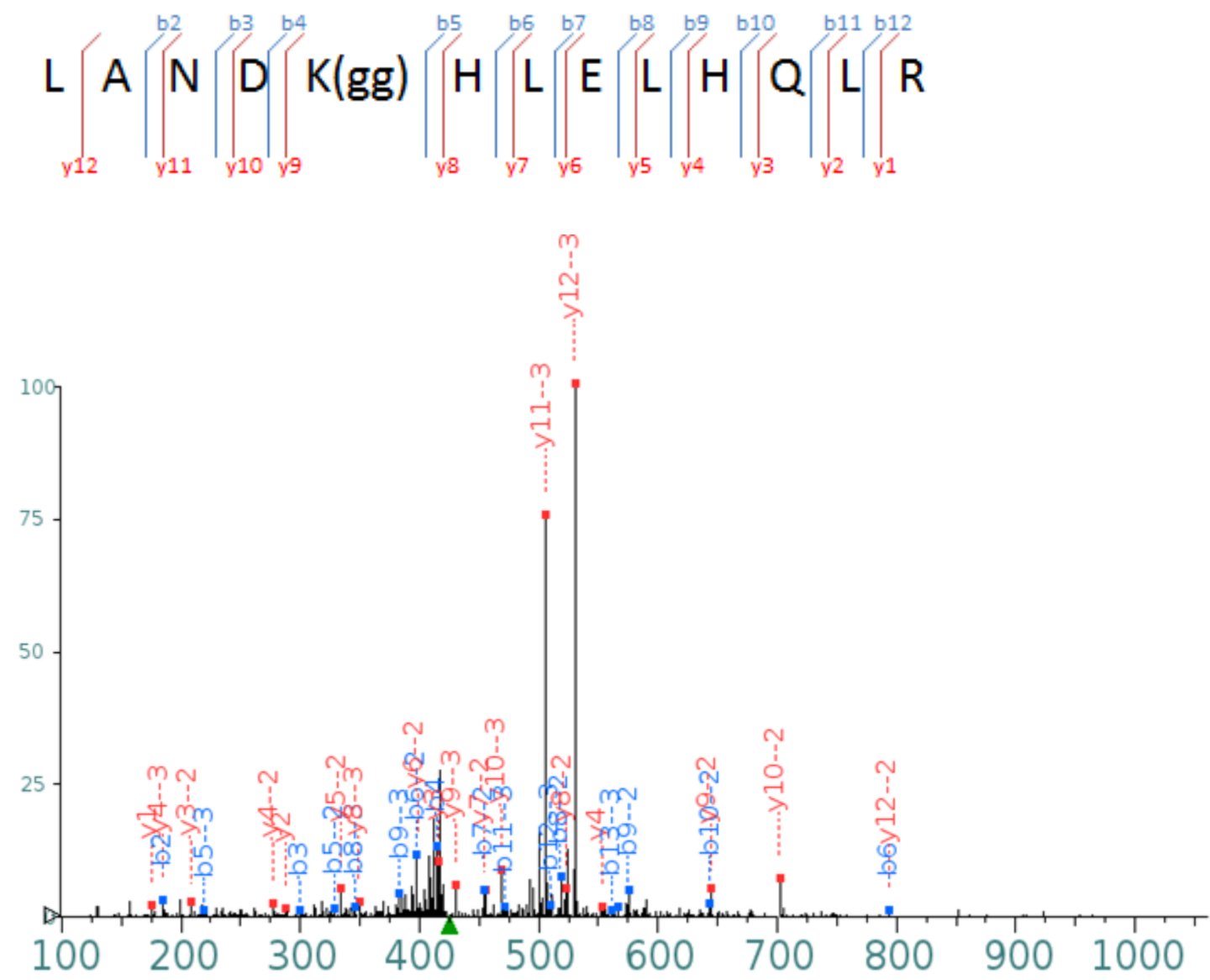

Fig. 18A

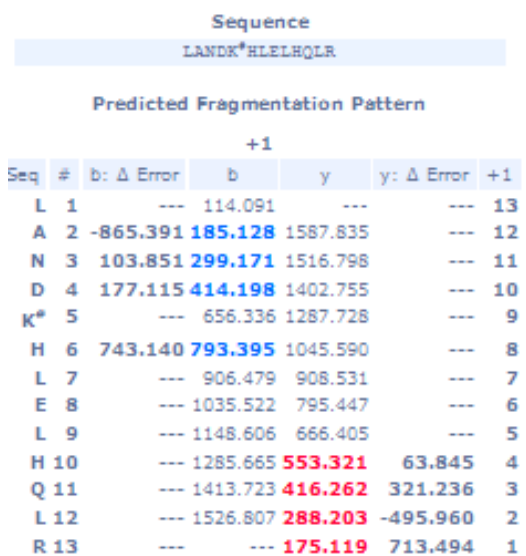

\begin{tabular}{|c|c|c|c|c|c|c|}
\hline \multicolumn{7}{|c|}{ Sequence } \\
\hline \multicolumn{7}{|c|}{ LANDK'HLELHOLR } \\
\hline \multicolumn{7}{|c|}{ Predicted Frágmentation Pattern } \\
\hline & & & +2 & & & \\
\hline Seq & $=$ & $\mathrm{b}: \Delta$ Error & b & y & $y: \Delta$ Error & +1 \\
\hline $\mathbf{L}$ & 1 & $\cdots$ & 57.549 & $\cdots$ & -.. & 13 \\
\hline A & 2 & $\ldots$ & 93.068 & 794.421 & -549.099 & 12 \\
\hline $\mathbf{N}$ & 3 & $\ldots$ & 150.089 & 758.903 & $\ldots$ & 11 \\
\hline D & 4 & $\cdots$ & 207.603 & 701.881 & 800.226 & 10 \\
\hline $\mathrm{K}^{*}$ & 5 & 2297.7213 & 328.672 & 644.368 & 299.075 & 9 \\
\hline H & 6 & 1794.3283 & 397.201 & 523.299 & 425.302 & 8 \\
\hline $\mathbf{L}$ & 7 & 233.9164 & 153.743 & 454.769 & 1842.685 & 7 \\
\hline $\mathbf{E}$ & 8 & 736.6805 & 518.265 & 398.227 & -784.157 & 6 \\
\hline $\mathbf{L}$ & 9 & 875.8115 & 574.807 & 333.706 & 1145.140 & 5 \\
\hline H 1 & & 213.6736 & 43.336 & 277.164 & 132.069 & 4 \\
\hline$Q 1$ & & $\cdots$ & 707.365 & 208.634 & 308.370 & 3 \\
\hline L 1 & & $\cdots$ & 763.907 & 144.605 & $\cdots$ & 2 \\
\hline R 1 & & $\ldots$ & $\cdots$ & 88.063 & $\cdots$ & 1 \\
\hline
\end{tabular}

\begin{tabular}{|c|c|c|c|c|c|c|}
\hline \multicolumn{7}{|c|}{ Sequence } \\
\hline \multicolumn{7}{|c|}{ LANDK' HLELHOLR } \\
\hline \multicolumn{7}{|c|}{ Predicted Fragmentation Pattern } \\
\hline & & & +3 & & & \\
\hline Seq $=$ & $=1$ & b: $\Delta$ Error & b & y & y: $\Delta$ Error & +1 \\
\hline L & 1 & - & 38.702 & $\cdots$ & - & 13 \\
\hline A & 2 & - & 62.381 & 529.950 & 597.564 & 12 \\
\hline N & 3 & - & 100.395 & 506.271 & 392.436 & 11 \\
\hline D & 4 & -. & 138.738 & 468.257 & 339.223 & 10 \\
\hline $\mathrm{K}^{*}$ & 5 & -1183.437 & 219.450 & 429.914 & 996.399 & 9 \\
\hline H & 6 & & 265.137 & 349.202 & 2482.337 & 8 \\
\hline L & 7 & & 302.8313 & 303.515 & - & 7 \\
\hline E : & 8 & 329.509 & 345.8452 & 265.821 & - & 6 \\
\hline L & 9 & -1111.774 & 383.5402 & 222.806 & - & 5 \\
\hline H 10 & & - & 429.226 & 185.112 & -774.739 & 4 \\
\hline Q 11 & & -1085.401 & 471.9131 & 139.425 & - & 3 \\
\hline L 12 & & 357.467 & 509.607 & 96.739 & - & 2 \\
\hline R 13 & & - & $\cdots$ & 59.045 & - & 1 \\
\hline
\end{tabular}

Fig. 18B 
Fig. 18. AnkB is ubiquitinated on lysine 67. The band at $\sim 74 \mathrm{kDa}$ (AnkB +6 ubiquitin) was analyzed by mass spectrometry and the resulting spectra within the ubiquitinated AnkB peptide (63-75) is shown with the b (blue lines) and y ions (red lines). (B) The fragmentation pattern of the $+1,+2$ and +3 charges of the $b$ and $y$ ions within the AnkB peptide $(63-75)$.

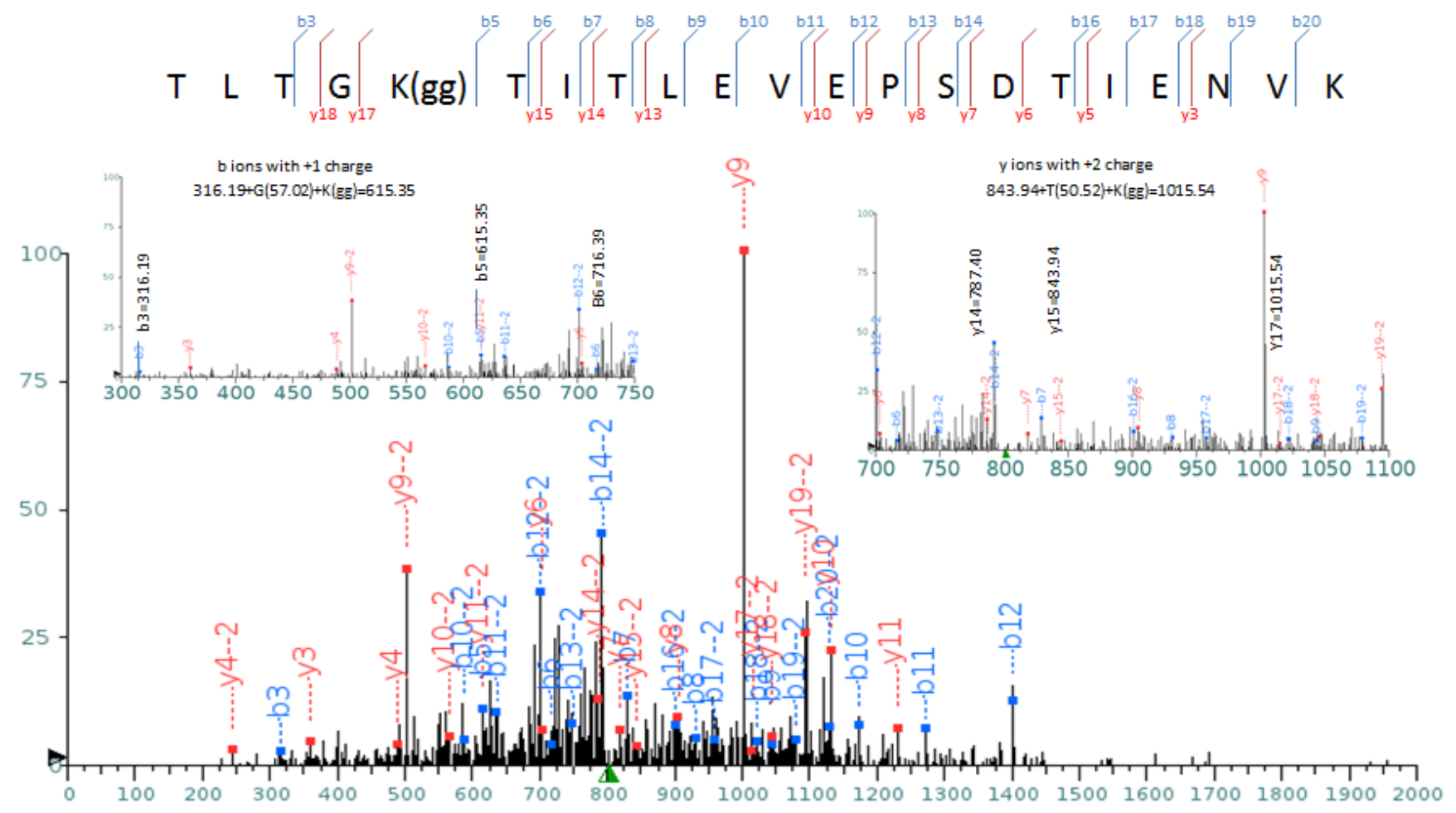

Fig. 19A 
Sequence TLTGK*TITLEVEPSTIENR:

Predicted Fragmentation Pattern

\begin{tabular}{|c|c|c|c|c|c|}
\hline & & + & & & \\
\hline Seq \# & $b: \Delta$ Error & b & $y$ & $y: \Delta$ Error & +1 \\
\hline T 1 & $\cdots$ & 102.055 & $\cdots$ & $\cdots$ & 21 \\
\hline L 2 & $\cdots$ & 215.139 & 2301.218 & $\cdots$ & 20 \\
\hline T 3 & 5.821 & 316.187 & 2186.134 & $\cdots$ & 19 \\
\hline G 4 & $\ldots$ & 373.208 & 2087.087 & $\cdots$ & 18 \\
\hline$K^{*} 5$ & 85.122 & 615.346 & 2030.065 & $\cdots$ & 17 \\
\hline T 6 & 256.643 & 716.394 & 1787.927 & $\ldots$ & 16 \\
\hline I 7 & -196.005 & 829.478 & 1686.850 & $\ldots$ & 15 \\
\hline T 8 & 806.436 & 930.525 & 1573.796 & $\cdots$ & 14 \\
\hline L 9 & -432.663 & 1043.610 & 1472.748 & $\cdots$ & 13 \\
\hline E 10 & 162.235 & 1172.652 & 1359.664 & $\cdots$ & 12 \\
\hline v 11 & 211.158 & 1271.721 & 1230.621 & 10.310 & 11 \\
\hline E 12 & 115.312 & 1400.763 & 1131.553 & 66.598 & 10 \\
\hline P 13 & $\ldots$ & 1497.816 & 1002.510 & 98.048 & 9 \\
\hline S 14 & $\cdots$ & 1584.848 & 905.457 & -1026.256 & 8 \\
\hline D 15 & $\cdots$ & 1699.875 & 818.425 & 206.895 & 7 \\
\hline T 16 & $\cdots$ & 1800.923 & 703.398 & 167.206 & 6 \\
\hline I 17 & $\cdots$ & 1914.007 & 602.351 & $\cdots$ & 5 \\
\hline E 18 & $\cdots$ & 2043.049 & 489.267 & -1862.492 & 4 \\
\hline N 19 & $\cdots$ & 2157.092 & 360.224 & 916.759 & 3 \\
\hline v 20 & $\cdots$ & 2256.161 & 246.181 & $\cdots$ & 2 \\
\hline K 21 & $\cdots$ & $\cdots$ & 147.113 & $\cdots$ & 1 \\
\hline
\end{tabular}

Sequence

TLTGK'TITLEVEPBTIENR

Predicted Fragmentation Pattern

\begin{tabular}{|c|c|c|c|c|c|}
\hline & & +2 & & & \\
\hline Seq $\#$ & $b: \Delta$ Error & b & $y$ & $y: \Delta$ Error & +1 \\
\hline T 1 & $\cdots$ & 51.531 & $\cdots$ & $\cdots$ & 21 \\
\hline L 2 & $\cdots$ & 108.073 & 1151.113 & $\cdots$ & 20 \\
\hline T 3 & $\cdots$ & 158.597 & 1094.571 & 153.093 & 19 \\
\hline G 4 & $\cdots$ & 187.108 & 1044.047 & 752.896 & 18 \\
\hline $\mathrm{K}^{*} \mathbf{5}$ & $\cdots$ & 308.177 & 1015.536 & -681.273 & 17 \\
\hline T 6 & $\cdots$ & 358.701 & 894.467 & $\cdots$ & 16 \\
\hline I 7 & $\cdots$ & 415.243 & 843.943 & 707.057 & 15 \\
\hline T 8 & $\cdots$ & 465.766 & 787.401 & .924 & 14 \\
\hline L 9 & $\cdots$ & 522.308 & 736.878 & $\cdots$ & 13 \\
\hline E 10 & 330.235 & 586.830 & 680 & $\cdots$ & 12 \\
\hline V 11 & -939.702 & 636.364 & 615.814 & -675.680 & 11 \\
\hline E 12 & 342.185 & 700.885 & 566.280 & 182.311 & 10 \\
\hline P 13 & -1205.642 & 749.412 & 501.759 & 672.194 & 9 \\
\hline S 14 & -463.667 & 792.928 & 453.232 & $\cdots$ & 8 \\
\hline D 15 & $\cdots$ & 850.441 & 409.716 & $\cdots$ & 7 \\
\hline T 16 & 365.404 & 900.965 & 352.203 & $\cdots$ & 6 \\
\hline I 17 & 429.971 & 957.507 & 301.679 & $\cdots$ & 5 \\
\hline E 18 & -372.176 & 1022.028 & 245.137 & -3746.872 & 4 \\
\hline N 19 & -149.147 & 1079.050 & 180.616 & $\cdots$ & 3 \\
\hline V 20 & 825.741 & 1128.584 & 123.594 & $\cdots$ & 2 \\
\hline K 21 & $\cdots$ & $\cdots$ & 74.060 & $\cdots$ & 1 \\
\hline
\end{tabular}

Fig. 19B

Fig. 19. AnkB is polyubiquitinated through lysine ${ }^{11}$ of ubiquitin. (A) The previously analyzed $\sim 74 \mathrm{kDa}$ ubiquitinated AnkB band was analyzed by mass spectrometry for ubiquitination of ubiquitin. The resulting spectrum of the modified ubiquitin peptide (727 ) is shown along with the $\mathrm{b}$ (blue lines) and $\mathrm{y}$ ions (red lines) detected. The $\mathrm{b}$ ions with $\mathrm{a}+1$ charge and $\mathrm{y}$ ions with $\mathrm{a}+2$ charge are shown with the mass/charge ratio calculated to show ubiquitination on lysine 11 of ubiquitin. (B) The fragmentation pattern of the $b$ and $y$ ions with $\mathrm{a}+1$ and +2 charge within the ubiquitin peptide (7-27). 


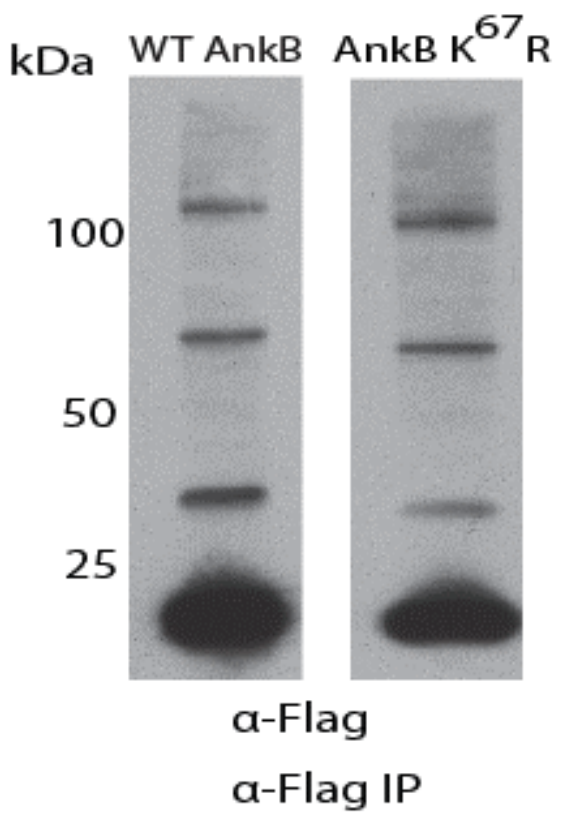

Fig. 20. Substitution of AnkB $K^{67}$ does not inhibit polyubiquitination. The

ubiquitinated lysine residue in $A n k B$ was substituted with an arginine $\left(A n k B K^{67} R\right)$ and transfected into HEK293T cells. Cell lysates were purified with anti-Flag agarose and analyzed by immunoblotting with anti-Flag antibodies. 


\section{Discussion Section 3}

Ubiquitination of eukaryotic proteins results in a myriad of biological consequences, such as proteasomal degradation, alteration in subcellular localization, signaling cascades and DNA repair [202, 209]. Intracellular bacteria have developed ingenious mechanisms to manipulate eukaryotic ubiquitination machinery for their own benefit [231, 235]. This manipulation can come from bacterial effectors that act as deubiquitinases, E3 ubiquitin ligases and F-box proteins that function identical to their eukaryotic counterpart [223, 231, 261]. F-box proteins, such as AnkB, typically cause proteasomal degradation of substrate proteins [279]. The substrates of AnkB are polyubiquitinated through $\mathrm{K}^{48}$-linkages and are degraded by the proteasomes, however, these targets are unknown [86]. While it has been shown that F-box proteins undergo autoubiquitination, which results in their proteasomal degradation, modification of F-box proteins through polyubiquitin chain linkages other than $\mathrm{K}^{48}$ has not been shown [280]. Autoubiquitination of F-box proteins is primarily a control mechanism to limit constant degradation of their substrates and unwanted protein turnover. Therefore, ubiquitination of F-box proteins resulting in proteasome-independent functions would be novel. Perhaps F-box proteins are ubiquitinated by non $\mathrm{K}^{48}$-linked polyubiquitination to interact with a specific substrate, or it could repress the downstream signaling events that occur through F-box mediated protein-protein interactions. F-box proteins regulate a wide array of cellular functions through proteasomal degradation of their substrates, so it could be easily perceived that ubiquitination of the F-box protein could alter this regulation allowing more possibilities for their cellular function.

Bacterial effectors have been shown to undergo ubiquitination during infection,

however the majority are $\mathrm{K}^{48}$-linked polyubiquitin chain linkages, which lead to proteasomal 
degradation [231, 281]. Detection of effector ubiquitination can be challenging, as a specific antibody to the effector is needed or a tag, such as HA is needed to perform immunoprecipitation. Fusing a tag to the effector can affect the translocation or alter the functions of the protein in vivo. Therefore, bacterial effectors could be modified through ubiquitination more commonly than has been shown in the literature due to the technical difficulties. The Salmonella effector SopB has been shown to be ubiquitinated through $\mathrm{K}^{63}$ linked polyubiquitination, which alters the subcellular localization during infection, while SopE and SptP are ubiquitinated and degraded by the proteasome [234, 282]. These examples highlight the ability of bacterial effector proteins to be ubiquitinated by the host cell machinery with biochemical consequences on their location, stability, or function.

The Philadelphia strain of L. pneumophila contains five F-box proteins, of which two were shown to be ubiquitinated during ectopic expression [228]. One of these F-box proteins shown to be ubiquitinated was LegAU13, which is the homologue to AnkB in the Philadelphia strain [228]. Interestingly, LegAU13 in the Philadelphia strain is not required for intracellular replication in human macrophages or amoeba [228], while AnkB in the AA100/130b strain is required for intracellular replication [131]. Furthermore, LegAU13 in the Philadelphia strain of L. pneumophila does not cause polyubiquitination of the LCV, which is mediated by another L. pneumophila effector SidC [261]. However, the SidC mediated polyubiquitination of the LCV is not required for intracellular replication. It has been shown that LegAU13 directly interacts with Skp1 and Cul1 of the SCF E3 ubiquitin ligase complex, however the targets are unknown [228]. Therefore, this diversity in a single effector protein reveals how L. pneumophila has a multitude of mechanisms that give rise to intracellular replication. Unfortunately, it was not shown if LegAU13 is ubiquitinated in the 
absence of the F-box domain, therefore it is not known if this ubiquitination is due to autoubiquitination in the Philadelphia strain. However, due to the ubiquitination of the Paris strain AnkB in a non-degradative manner, it is likely the same occurs for the Philadelphia strain AnkB as well. This raises an intriguing question as to how different $L$. pneumophila strains utilize the effector proteins they have acquired. While this dissertation focuses on AnkB, there is a multitude of effector proteins that allow L.pneumophila to avoid phagasome-lysosome fusion and the formation of the LCV which provides the platform for AnkB to function upon.

The $\mathrm{K}^{11}$-linked polyubiquitination of AnkB introduces two novel findings to cellular biology. It is the first bacterial effector protein shown to be polyubiquitinated through $\mathrm{K}^{11}$ linkages, and the first prokaryotic or eukaryotic F-box protein shown to be polyubiquitinated through $\mathrm{K}^{11}$ linkages. Interestingly, $\mathrm{K}^{11}$-linked polyubiquitination of $\mathrm{AnkB}$ does not lead to proteasomal degradation. Unlike other examples of $\mathrm{K}^{11}$-linked polyubiquitination, the stability of AnkB is not affected. Unlike other examples of $\mathrm{K}^{11}$-linked polyubiquitination that have primarily been seen by proteins involved in the cell cycle regulation through ubiquitination by the Anaphase -promoting complex E3 ubiquitin ligase, AnkB is a bacterial effector that lacks these properties [283, 284]. This atypical $\mathrm{K}^{11}$-linked ubiquitination of a bacterial effector raises the question if other effectors are modified through similar polyubiquitin chain linkages. We have observed the ubiquitination of AnkB on several different $\mathrm{K}$ residues through mass spectrometry, and the $\mathrm{K}$ residues were located in the $\mathrm{C}$ terminus and $\mathrm{N}$ terminus of the protein. It has been shown for bacterial effectors as well as eukaryotic proteins that ubiquitination can occur on multiple $\mathrm{K}$ residues and there is little specificity as to which $\mathrm{K}$ is modified [234, 278]. When two of the ubiquitinated $\mathrm{K}$ residues of 
AnkB were substituted with arginine residues, ubiquitination of AnkB still occurred (though likely on other $\mathrm{K}$ residues). Therefore, it was not possible to determine if the ubiquitination is needed for the functional aspects of AnkB or for intracellular replication of L. pneumophila. AnkB contains $19 \mathrm{~K}$ residues, therefore we did not proceed with the substitution as we were concerned that substitution of 19 residues could lead to the protein function being compromised. However, if we could determine that the ubiquitination occurs in a specific region or domain of $\mathrm{AnkB}$, this substitution procedure would be more accurate. Determining which eukaryotic E3 ubiquitin ligases are able to recognize bacterial proteins, and how this recognition can occur, should strengthen the information on effector ubiquitination. The $\mathrm{K}^{48}$ linked polyubiquitination of effectors can be explained as being a foreign protein, therefore the E3 ubiquitin ligases marks the protein for proteasomal degradation. The mechanisms underlying polyubiquitination of effector proteins independent of proteasomal degradation is nonexistent.

There is limited knowledge on ubiquitination other than $\mathrm{K}^{48}$ or $\mathrm{K}^{63}$, which includes $\left(\mathrm{K}^{6}, \mathrm{~K}^{11}, \mathrm{~K}^{27}, \mathrm{~K}^{29}, \mathrm{~K}^{33}\right)$. Importantly, the $\mathrm{K}^{11}$-linked polyubiquitination of AnkB shows another mode of ubiquitination of bacterial effector proteins independent of proteasomal degradation. The ubiquitination of AnkB could result in a-conformational change, therefore causing AnkB to release the substrates of the ankyrin domains. This type of signaling has been seen with $\mathrm{K}^{29}$-linked polyubiquitination [285]. It does not appear that this ubiquitination would affect the subcellular localization of AnkB, as it is anchored into the LCV membrane through host-mediated farnesylation immediately following translocation $[135,241]$. The ubiquitination of AnkB most likely occurs on the LCV during infection, however the timing of this is yet to be determined. Ubiquitination 
of SopB from Salmonella does not occur until $\sim 1$ hour after infection, therefore in vivo ubiquitination analysis should be evaluated at multiple time points during infection [234]. The function of AnkB during infection is to provide an increase in free cellular amino acids, which is essential to support replication. Therefore the absence of proteasomal degradation during ectopic expression of AnkB supports the hypothesis that AnkB is functional throughout the infection. Further research into the mechanisms of this $\mathrm{K}^{11}$ linked polyubiquitination will answer unknown questions regarding this mode of polyubiquitination. Therefore, while the function is unknown, this represents novel polyubiquitination of an F-box protein, and could present valuable information on the function of F-box proteins 


\section{CONCLUSIONS AND FUTURE DIRECTIONS}

This project has determined the initial kinetics and mechanisms of the

L. pneumophila effector AnkB upon intimate contact of extracellular bacteria with the eukaryotic host. AnkB is the only one of seven eukaryotic-like ankyrin proteins in L. pneumophila to be translocated from attached extracellular bacteria. This shows an unique ability for a bacterium to prioritize the translocation of certain effectors, probably the effector proteins that are needed for early aspects of intracellular replication. We conclude that AnkB is translocated from attached extracellular bacteria to generate a surplus of amino acids that prevents differentiation from the replicative phase to the transmissive phase and will power subsequent intracellular replication. This pre-programming of the nutrient sources in the host cell by AnkB shows a level of sophistication L. pneumophila has obtained to generate a favorable nutrient rich environment within human macrophages and amoeba.

The recruitment of the farnesylation and ubiquitination machinery is dependent on the Dot/Icm secretion system; AnkB appears to play a critical role in this recruitment as the ankB mutant strain is less efficient at recruitment compared to the WT strain. While

L. pneumophila is the only intracellular pathogen known to recruit host cell farnesylation and polyubiquitination machineries to the point of extracellular bacterial attachment, undoubtedly, other intracellular pathogens manipulate host cellular processes during the initial infection process to establish a suitable niche for intracellular replication. 
The polyubiquitinated proteins on the LCV have a wide range of cellular processes, including immune response and nutrient transport. L. pneumophila encodes at least 2 E3 ubiquitin ligases, which could potentially ubiquitinate some of the immune response proteins included in the ubiquitinated proteome, causing their proteasomal degradation and thereby influencing the immune response of the cell. To determine the substrates of AnkB is an ongoing process that will identify specific eukaryotic proteins that are degraded by the proteasomes to generate a surplus of amino acids. Ankyrin domains have the ability to bind more than one interacting protein. Therefore it is unknown how many substrates AnkB has or if the substrates are proteins expressed in high abundance. The ubiquitinated proteome can be utilized in RNAi knockdown experiments of the identified proteins, which can help distinguish which ubiquitinated proteins are required for intracellular replication. The unknown ubiquitinated targets of AnkB are most likely in the ubiquitinated proteome of the WT strain and therefore we have reduced the amount of possible binding partners from the $\sim 250$ proteins localized to WT strain LCV's and excluded from the ankB mutant strain LCV. The $\mathrm{K}^{48}$-linked polyubiquitinated substrates of AnkB are degraded by the proteasomes, therefore another possibility is that proteins found on the ankB mutant strain LCV and excluded on the WT strain LCV could be the AnkB substrates. However, due to the published confocal microscopy data showing the cloud of polyubiquitinated proteins surrounding the LCV throughout infection, we favor the former possibility. The E2 ubiquitinconjugating enzymes and the E3 ubiquitin ligases in the complete LCV proteome can help decipher the complexity of the LCV ubiquitination and perhaps determine additional host cell ubiquitination machinery that is required for infection, which was previously shown for the SCF1 E3 ubiquitin ligase complex. Knockdown experiments using RNAi on the E3 ubiquitin 
ligases and the E2 ubiquitin-conjugating enzymes can determine which are needed and possibly manipulated by L. pneumophila during infection. Furthermore, the identified E3 ubiquitin ligases can polyubiquitinate L. pneumophila effectors leading to their proteasomal degradation, altered sub-cellular localization or function.

To date we have not been able to inhibit the ubiquitination of AnkB by substituting the ubiquitinated lysine residue with an arginine residue. Therefore, we have not been able to determine the effect of the lysine ${ }^{11}$-linked polyubiquitination of AnkB. However, the polyubiquitination of AnkB does not result in its proteasomal degradation. As AnkB is the first F-box protein shown to be modified through lysine ${ }^{11}$-linked polyubiquitination, determining the mechanism could add valuable evidence into the field of lysine ${ }^{11}$ polyubiquitination. Lysine ${ }^{11}$-linked polyubiquitination of AnkB could possible disrupt the binding between the ankyrin domains of AnkB and their target proteins or possibly cause some downstream signaling events to occur. Future studies will be to substitute multiple lysine residues in AnkB with arginine residues, and perform the same immunoprecipitation experiments to determine if lack of ubiquitination changes the function of AnkB. This substitution method was performed with the Salmonella effector SopB, where they noticed SopB was polyubiquitinated through $\mathrm{K}^{63}$ and the result was an altered subcellular localization[234]. Understanding the mechanism of the lysine ${ }^{11}$-linked polyubiquitination will provide information on effector protein polyubiquitination, which is a field with only a few examples. While AnkB specifically interacts with Trim21 it has not been experimentally verified that Trim 21 ubiquitinates AnkB. Using siRNA against Trim21 did not abolish the ubiquitination of AnkB, therefore we could not conclude Trim21 ubiquitinates AnkB (data not shown). However, technical issues with anti-Trim21 antibodies 
possibly played a role in determining efficient knockdown. Therefore, future experiments would look into the AnkB-Trim21 interaction and determine if Trim21 is the E3 ubiquitin ligase responsible for the ubiquitination of AnkB. This can be achieved by immunoprecipitating AnkB from Trim21 knockout cells and detecting ubiquitination of AnkB by Western Blot or more specific mass spectrometry. To determine if Trim21 is a substrate of AnkB we can perform co-immunoprecipitation experiments with an AnkB variant lacking the ankyrin domains and probe the Western Blot membrane with anti-Trim21. These future experiments will help solidify the mechanism AnkB utilizes during intracellular infection to provide the gratuitous supply of amino acids. 


\section{REFERENCES}

1. McDade, J.E., et al., Legionnaires' disease: isolation of a bacterium and demonstration of its role in other respiratory disease. N Engl J Med, 1977. 297(22): p. 1197-203.

2. $\quad$ Fraser, D.W., et al., Legionnaires' disease: description of an epidemic of pneumonia. N Engl J Med, 1977. 297(22): p. 1189-97.

3. $\quad$ Fry, N.K., et al., The use of $16 \mathrm{~S}$ ribosomal RNA analyses to investigate the phylogeny of the family Legionellaceae. J Gen Microbiol, 1991. 137(5): p. 121522.

4. $\quad$ Fry, N.K., et al., Direct amplification and sequencing of the $16 S$ ribosomal DNA of an intracellular Legionella species recovered by amoebal enrichment from the sputum of a patient with pneumonia. FEMS Microbiol Lett, 1991. 67(2): p. 165-8.

5. Palusinska-Szysz, M. and M. Cendrowska-Pinkosz, Pathogenicity of the family Legionellaceae. Arch Immunol Ther Exp (Warsz), 2009. 57(4): p. 279-90.

6. Brenner, D.J., Classification of the legionellae. Semin Respir Infect, 1987. 2(4): p. 190-205.

7. Benson, R.F. and B.S. Fields, Classification of the genus Legionella. Semin Respir Infect, 1998. 13(2): p. 90-9.

8. Fields, B.S., R.F. Benson, and R.E. Besser, Legionella and Legionnaires' disease: 25 years of investigation. Clin Microbiol Rev, 2002. 15(3): p. 506-26.

9. Den Boer, J.W. and E.P. Yzerman, Diagnosis of Legionella infection in Legionnaires' disease. Eur J Clin Microbiol Infect Dis, 2004. 23(12): p. 871-8.

10. Marston, B.J., H.B. Lipman, and R.F. Breiman, Surveillance for Legionnaires' disease. Risk factors for morbidity and mortality. Arch Intern Med, 1994. 154(21): p. 2417-22.

11. O'Connor, B.A., et al., Does using potting mix make you sick? Results from a Legionella longbeachae case-control study in South Australia. Epidemiol Infect, 2007. 135(1): p. 34-9.

12. Yu, V.L., et al., Distribution of Legionella species and serogroups isolated by culture in patients with sporadic community-acquired legionellosis: an international collaborative survey. J Infect Dis, 2002. 186(1): p. 127-8.

13. Abu Kwaik, Y., et al., Invasion of protozoa by Legionella pneumophila and its role in bacterial ecology and pathogenesis. Appl Environ Microbiol, 1998. 64(9): p. 3127-33.

14. Fields, B.S., The molecular ecology of legionellae. Trends Microbiol, 1996. 4(7): p. 286-90.

15. Breiman, R.F., et al., Role of air sampling in investigation of an outbreak of legionnaires' disease associated with exposure to aerosols from an evaporative condenser. J Infect Dis, 1990. 161(6): p. 1257-61. 
16. Steinert, M., U. Hentschel, and J. Hacker, Legionella pneumophila: an aquatic microbe goes astray. FEMS Microbiol Rev, 2002. 26(2): p. 149-62.

17. Den Boer, J.W., et al., A large outbreak of Legionnaires' disease at a flower show, the Netherlands, 1999. Emerg Infect Dis, 2002. 8(1): p. 37-43.

18. Winn, W.C., Jr., Legionnaires disease: historical perspective. Clin Microbiol Rev, 1988. 1(1): p. 60-81.

19. Chastre, J., et al., Pulmonary fibrosis following pneumonia due to acute Legionnaires' disease. Clinical, ultrastructural, and immunofluorescent study. Chest, 1987. 91(1): p. 57-62.

20. Kaufmann, A.F., et al., Pontiac fever: isolation of the etiologic agent (Legionella pneumophilia) and demonstration of its mode of transmission. Am J Epidemiol, 1981. 114(3): p. 337-47.

21. Tossa, P., et al., Pontiac fever: an operational definition for epidemiological studies. BMC Public Health, 2006. 6: p. 112.

22. Burnsed, L.J., et al., A large, travel-associated outbreak of legionellosis among hotel guests: utility of the urine antigen assay in confirming Pontiac fever. Clin Infect Dis, 2007. 44(2): p. 222-8.

23. Cosentini, R., et al., Community-acquired pneumonia: role of atypical organisms. Monaldi Arch Chest Dis, 2001. 56(6): p. 527-34.

24. Luck, P.C. and M. Steinert, [Pathogenesis, diagnosis and therapy of Legionella infections]. Bundesgesundheitsblatt Gesundheitsforschung Gesundheitsschutz, 2006. 49(5): p. 439-49.

25. Sabria, M. and M. Campins, Legionnaires' disease: update on epidemiology and management options. Am J Respir Med, 2003. 2(3): p. 235-43.

26. Guyot, S., et al., Legionella pneumophila aortitis in a heart transplant recipient. Transpl Infect Dis, 2007. 9(1): p. 58-9.

27. Mathys, W., et al., Fatal nosocomial Legionnaires' disease after heart transplantation: clinical course, epidemiology and prevention strategies for the highly immunocompromized host. J Hosp Infect, 1999. 43(3): p. 242-6.

28. Carratala, J., et al., Risk factors for nosocomial Legionella pneumophila pneumonia. Am J Respir Crit Care Med, 1994. 149(3 Pt 1): p. 625-9.

29. Fliermans, C.B., et al., Ecological distribution of Legionella pneumophila. Appl Environ Microbiol, 1981. 41(1): p. 9-16.

30. Doebbeling, B.N. and R.P. Wenzel, The epidemiology of Legionella pneumophila infections. Semin Respir Infect, 1987. 2(4): p. 206-21.

31. Costerton, J.W., et al., Microbial biofilms. Annu Rev Microbiol, 1995. 49: p. 71145.

32. Stickler, D., Biofilms. Curr Opin Microbiol, 1999. 2(3): p. 270-5.

33. Kilvington, S. and J. Price, Survival of Legionella pneumophila within cysts of Acanthamoeba polyphaga following chlorine exposure. J Appl Bacteriol, 1990. 68(5): p. 519-25.

34. Rowbotham, T.J., Current views on the relationships between amoebae, legionellae and man. Isr J Med Sci, 1986. 22(9): p. 678-89.

35. Barker, J., H. Scaife, and M.R. Brown, Intraphagocytic growth induces an antibiotic-resistant phenotype of Legionella pneumophila. Antimicrob Agents Chemother, 1995. 39(12): p. 2684-8. 
36. Barker, J., et al., Relationship between Legionella pneumophila and Acanthamoeba polyphaga: physiological status and susceptibility to chemical inactivation. Appl Environ Microbiol, 1992. 58(8): p. 2420-5.

37. Cirillo, J.D., S. Falkow, and L.S. Tompkins, Growth of Legionella pneumophila in Acanthamoeba castellanii enhances invasion. Infect Immun, 1994. 62(8): p. 3254-61.

38. Cirillo, J.D., et al., Intracellular growth in Acanthamoeba castellanii affects monocyte entry mechanisms and enhances virulence of Legionella pneumophila. Infect Immun, 1999. 67(9): p. 4427-34.

39. Garduno, R.A., E. Garduno, and P.S. Hoffman, Surface-associated hsp60 chaperonin of Legionella pneumophila mediates invasion in a HeLa cell model. Infect Immun, 1998. 66(10): p. 4602-10.

40. O'Brien, S.J. and R.S. Bhopal, Legionnaires' disease: the infective dose paradox. Lancet, 1993. 342(8862): p. 5-6.

41. Rowbotham, T.J., Preliminary report on the pathogenicity of Legionella pneumophila for freshwater and soil amoebae. J Clin Pathol, 1980. 33(12): p. 1179-83.

42. abu Kwaik, Y., B.S. Fields, and N.C. Engleberg, Protein expression by the protozoan Hartmannella vermiformis upon contact with its bacterial parasite Legionella pneumophila. Infect Immun, 1994. 62(5): p. 1860-6.

43. Payne, N.R. and M.A. Horwitz, Phagocytosis of Legionella pneumophila is mediated by human monocyte complement receptors. J Exp Med, 1987. 166(5): p. 1377-89.

44. King, C.H., et al., Effects of cytochalasin D and methylamine on intracellular growth of Legionella pneumophila in amoebae and human monocyte-like cells. Infect Immun, 1991. 59(3): p. 758-63.

45. Harb, O.S., et al., Heterogeneity in the attachment and uptake mechanisms of the Legionnaires' disease bacterium, Legionella pneumophila, by protozoan hosts. Appl Environ Microbiol, 1998. 64(1): p. 126-32.

46. Venkataraman, C., et al., Identification of a Gal/GalNAc lectin in the protozoan Hartmannella vermiformis as a potential receptor for attachment and invasion by the Legionnaires' disease bacterium. J Exp Med, 1997. 186(4): p. 537-47.

47. Venkataraman, C., et al., Identification of putative cytoskeletal protein homologues in the protozoan host Hartmannella vermiformis as substrates for induced tyrosine phosphatase activity upon attachment to the Legionnaires' disease bacterium, Legionella pneumophila. J Exp Med, 1998. 188(3): p. 505-14.

48. Bozue, J.A. and W. Johnson, Interaction of Legionella pneumophila with Acanthamoeba castellanii: uptake by coiling phagocytosis and inhibition of phagosome-lysosome fusion. Infect Immun, 1996. 64(2): p. 668-73.

49. Horwitz, M.A., Phagocytosis of the Legionnaires' disease bacterium (Legionella pneumophila) occurs by a novel mechanism: engulfment within a pseudopod coil. Cell, 1984. 36(1): p. 27-33.

50. Gao, L.Y., O.S. Harb, and Y. Abu Kwaik, Utilization of similar mechanisms by Legionella pneumophila to parasitize two evolutionarily distant host cells, mammalian macrophages and protozoa. Infect Immun, 1997. 65(11): p. 4738-46. 
51. Horwitz, M.A., Formation of a novel phagosome by the Legionnaires' disease bacterium (Legionella pneumophila) in human monocytes. J Exp Med, 1983. 158(4): p. 1319-31.

52. Abu Kwaik, Y., The phagosome containing Legionella pneumophila within the protozoan Hartmannella vermiformis is surrounded by the rough endoplasmic reticulum. Appl Environ Microbiol, 1996. 62(6): p. 2022-8.

53. Horwitz, M.A. and S.C. Silverstein, Legionnaires' disease bacterium (Legionella pneumophila) multiples intracellularly in human monocytes. J Clin Invest, 1980. 66(3): p. 441-50.

54. Nash, T.W., D.M. Libby, and M.A. Horwitz, Interaction between the legionnaires' disease bacterium (Legionella pneumophila) and human alveolar macrophages. Influence of antibody, lymphokines, and hydrocortisone. J Clin Invest, 1984. 74(3): p. 771-82.

55. Tilney, L.G., et al., How the parasitic bacterium Legionella pneumophila modifies its phagosome and transforms it into rough ER: implications for conversion of plasma membrane to the ER membrane. J Cell Sci, 2001. 114(Pt 24): p. 4637-50.

56. Kagan, J.C. and C.R. Roy, Legionella phagosomes intercept vesicular traffic from endoplasmic reticulum exit sites. Nat Cell Biol, 2002. 4(12): p. 945-54.

57. Swanson, M.S. and R.R. Isberg, Association of Legionella pneumophila with the macrophage endoplasmic reticulum. Infect Immun, 1995. 63(9): p. 3609-20.

58. Horwitz, M.A., The Legionnaires' disease bacterium (Legionella pneumophila) inhibits phagosome-lysosome fusion in human monocytes. J Exp Med, 1983. 158(6): p. 2108-26.

59. Horwitz, M.A. and F.R. Maxfield, Legionella pneumophila inhibits acidification of its phagosome in human monocytes. J Cell Biol, 1984. 99(6): p. 1936-43.

60. Sturgill-Koszycki, S. and M.S. Swanson, Legionella pneumophila replication vacuoles mature into acidic, endocytic organelles. J Exp Med, 2000. 192(9): p. 1261-72.

61. Gao, L.Y. and Y.A. Kwaik, The mechanism of killing and exiting the protozoan host Acanthamoeba polyphaga by Legionella pneumophila. Environ Microbiol, 2000. 2(1): p. 79-90.

62. Gao, L.Y. and Y. Abu Kwaik, Activation of caspase 3 during Legionella pneumophila-induced apoptosis. Infect Immun, 1999. 67(9): p. 4886-94.

63. Gao, L.Y. and Y. Abu Kwaik, Apoptosis in macrophages and alveolar epithelial cells during early stages of infection by Legionella pneumophila and its role in cytopathogenicity. Infect Immun, 1999. 67(2): p. 862-70.

64. Byrne, B. and M.S. Swanson, Expression of Legionella pneumophila virulence traits in response to growth conditions. Infect Immun, 1998. 66(7): p. 3029-34.

65. Zhu, W., et al., Induction of caspase 3 activation by multiple Legionella pneumophila Dot/Icm substrates. Cell Microbiol, 2013.

66. Molmeret, M., et al., Activation of caspase-3 by the Dot/Icm virulence system is essential for arrested biogenesis of the Legionella-containing phagosome. Cell Microbiol, 2004. 6(1): p. 33-48.

67. Abu-Zant, A., et al., Anti-apoptotic signalling by the Dot/Icm secretion system of L. pneumophila. Cell Microbiol, 2007. 9(1): p. 246-64. 
68. Abu-Zant, A., et al., Incomplete activation of macrophage apoptosis during intracellular replication of Legionella pneumophila. Infect Immun, 2005. 73(9): p. 5339-49.

69. Monroe, K.M., S.M. McWhirter, and R.E. Vance, Identification of host cytosolic sensors and bacterial factors regulating the type I interferon response to Legionella pneumophila. PLoS Pathog, 2009. 5(11): p. e1000665.

70. Kirby, J.E., et al., Evidence for pore-forming ability by Legionella pneumophila. Mol Microbiol, 1998. 27(2): p. 323-36.

71. Alli, O.A., et al., Temporal pore formation-mediated egress from macrophages and alveolar epithelial cells by Legionella pneumophila. Infect Immun, 2000. 68(11): p. 6431-40.

72. Molmeret, M., et al., The C-terminus of IcmT is essential for pore formation and for intracellular trafficking of Legionella pneumophila within Acanthamoeba polyphaga. Mol Microbiol, 2002. 43(5): p. 1139-50.

73. Urwyler, S., et al., Proteome analysis of Legionella vacuoles purified by magnetic immunoseparation reveals secretory and endosomal GTPases. Traffic, 2009. 10(1): p. 76-87.

74. Hoffmann, C., et al., Functional analysis of novel Rab GTPases identified in the proteome of purified Legionella-containing vacuoles from macrophages. Cell Microbiol, 2013.

75. Shevchuk, O., et al., Proteomic analysis of Legionella-containing phagosomes isolated from Dictyostelium. Int J Med Microbiol, 2009. 299(7): p. 489-508.

76. Salins, S., et al., Differential induction of gamma interferon in Legionella pneumophila-infected macrophages from BALB/c and $A / J$ mice. Infect Immun, 2001. 69(6): p. 3605-10.

77. Gebran, S.J., et al., Differences and similarities in permissive A/J versus nonpermissive BALB/c murine macrophages infected with Legionella pneumophila: the role of iron. FEMS Immunol Med Microbiol, 1994. 9(1): p. 7-14.

78. Kagan, J.C., et al., Legionella subvert the functions of Rab1 and Sec22b to create a replicative organelle. J Exp Med, 2004. 199(9): p. 1201-11.

79. Nagai, H., et al., A bacterial guanine nucleotide exchange factor activates ARF on Legionella phagosomes. Science, 2002. 295(5555): p. 679-82.

80. Weber, S.S., et al., Legionella pneumophila exploits PI(4)P to anchor secreted effector proteins to the replicative vacuole. PLoS Pathog, 2006. 2(5): p. e46.

81. Haneburger, I. and H. Hilbi, Phosphoinositide lipids and the Legionella pathogen vacuole. Curr Top Microbiol Immunol, 2013. 376: p. 155-73.

82. Finsel, I., et al., The Legionella effector RidL inhibits retrograde trafficking to promote intracellular replication. Cell Host Microbe, 2013. 14(1): p. 38-50.

83. Huang, L., et al., The E Block motif is associated with Legionella pneumophila translocated substrates. Cell Microbiol, 2011. 13(2): p. 227-45.

84. Tesh, M.J. and R.D. Miller, Amino acid requirements for Legionella pneumophila growth. J Clin Microbiol, 1981. 13(5): p. 865-9.

85. Wieland, H., et al., Intracellular multiplication of Legionella pneumophila depends on host cell amino acid transporter SLC1A5. Mol Microbiol, 2005. 55(5): p. 1528-37. 
86. Price, C.T., et al., Host proteasomal degradation generates amino acids essential for intracellular bacterial growth. Science, 2011. 334(6062): p. 1553-7.

87. Chien, M., et al., The genomic sequence of the accidental pathogen Legionella pneumophila. Science, 2004. 305(5692): p. 1966-8.

88. Eylert, E., et al., Isotopologue profiling of Legionella pneumophila: role of serine and glucose as carbon substrates. J Biol Chem, 2010. 285(29): p. 22232-43.

89. Al-Quadan, T., C.T. Price, and Y. Abu Kwaik, Exploitation of evolutionarily conserved amoeba and mammalian processes by Legionella. Trends Microbiol, 2012. 20(6): p. 299-306.

90. Price, C.T., A.M. Richards, and Y. Abu Kwaik, Nutrient generation and retrieval from the host cell cytosol by intra-vacuolar Legionella pneumophila. Front Cell Infect Microbiol, 2014. 4: p. 111.

91. Schunder, E., et al., Amino Acid Uptake and Metabolism of Legionella pneumophila Hosted by Acanthamoeba castellanii. J Biol Chem, 2014.

92. Harada, E., et al., Glucose metabolism in Legionella pneumophila: dependence on the Entner-Doudoroff pathway and connection with intracellular bacterial growth. J Bacteriol, 2010. 192(11): p. 2892-9.

93. Garduno, R.A., et al., Intracellular growth of Legionella pneumophila gives rise to a differentiated form dissimilar to stationary-phase forms. Infect Immun, 2002. 70(11): p. 6273-83.

94. Hammer, B.K. and M.S. Swanson, Co-ordination of legionella pneumophila virulence with entry into stationary phase by ppGpp. Mol Microbiol, 1999. 33(4): p. 721-31.

95. Molofsky, A.B. and M.S. Swanson, Differentiate to thrive: lessons from the Legionella pneumophila life cycle. Mol Microbiol, 2004. 53(1): p. 29-40.

96. Hammer, B.K., E.S. Tateda, and M.S. Swanson, A two-component regulator induces the transmission phenotype of stationary-phase Legionella pneumophila. Mol Microbiol, 2002. 44(1): p. 107-18.

97. Molofsky, A.B. and M.S. Swanson, Legionella pneumophila CsrA is a pivotal repressor of transmission traits and activator of replication. Mol Microbiol, 2003. 50(2): p. 445-61.

98. Heuner, K., et al., Influence of the alternative sigma(28) factor on virulence and flagellum expression of Legionella pneumophila. Infect Immun, 2002. 70(3): p. 1604-8.

99. Schulz, T., et al., FliA expression analysis and influence of the regulatory proteins RpoN, FleQ and FliA on virulence and in vivo fitness in Legionella pneumophila. Arch Microbiol, 2012. 194(12): p. 977-89.

100. Hales, L.M. and H.A. Shuman, Legionella pneumophila contains a type II general secretion pathway required for growth in amoebae as well as for secretion of the Msp protease. Infect Immun, 1999. 67(7): p. 3662-6.

101. Liles, M.R., V.K. Viswanathan, and N.P. Cianciotto, Identification and temperature regulation of Legionella pneumophila genes involved in type IV pilus biogenesis and type II protein secretion. Infect Immun, 1998. 66(4): p. 1776-82.

102. Rossier, O. and N.P. Cianciotto, Type II protein secretion is a subset of the PilDdependent processes that facilitate intracellular infection by Legionella pneumophila. Infect Immun, 2001. 69(4): p. 2092-8. 
103. Vogel, J.P., et al., Conjugative transfer by the virulence system of Legionella pneumophila. Science, 1998. 279(5352): p. 873-6.

104. Cianciotto, N.P., Many substrates and functions of type II secretion: lessons learned from Legionella pneumophila. Future Microbiol, 2009. 4(7): p. 797-805.

105. Johnson, T.L., et al., Type II secretion: from structure to function. FEMS Microbiol Lett, 2006. 255(2): p. 175-86.

106. Rossier, O., J. Dao, and N.P. Cianciotto, The type II secretion system of Legionella pneumophila elaborates two aminopeptidases, as well as a metalloprotease that contributes to differential infection among protozoan hosts. Appl Environ Microbiol, 2008. 74(3): p. 753-61.

107. Aragon, V., et al., Secreted enzymatic activities of wild-type and pilD-deficient Legionella pneumophila. Infect Immun, 2000. 68(4): p. 1855-63.

108. Banerji, S., et al., Characterization of the major secreted zinc metalloproteasedependent glycerophospholipid:cholesterol acyltransferase, PlaC, of Legionella pneumophila. Infect Immun, 2005. 73(5): p. 2899-909.

109. Aragon, V., S. Kurtz, and N.P. Cianciotto, Legionella pneumophila major acid phosphatase and its role in intracellular infection. Infect Immun, 2001. 69(1): p. 177-85.

110. Cianciotto, N.P., Type II secretion: a protein secretion system for all seasons. Trends Microbiol, 2005. 13(12): p. 581-8.

111. Rossier, O., S.R. Starkenburg, and N.P. Cianciotto, Legionella pneumophila type II protein secretion promotes virulence in the A/J mouse model of Legionnaires' disease pneumonia. Infect Immun, 2004. 72(1): p. 310-21.

112. Berger, K.H. and R.R. Isberg, Two distinct defects in intracellular growth complemented by a single genetic locus in Legionella pneumophila. Mol Microbiol, 1993. 7(1): p. 7-19.

113. Marra, A., et al., Identification of a Legionella pneumophila locus required for intracellular multiplication in human macrophages. Proc Natl Acad Sci U S A, 1992. 89(20): p. 9607-11.

114. Horwitz, M.A., Characterization of avirulent mutant Legionella pneumophila that survive but do not multiply within human monocytes. J Exp Med, 1987. 166(5): p. 1310-28.

115. Brand, B.C., A.B. Sadosky, and H.A. Shuman, The Legionella pneumophila icm locus: a set of genes required for intracellular multiplication in human macrophages. Mol Microbiol, 1994. 14(4): p. 797-808.

116. Berger, K.H., J.J. Merriam, and R.R. Isberg, Altered intracellular targeting properties associated with mutations in the Legionella pneumophila dotA gene. Mol Microbiol, 1994. 14(4): p. 809-22.

117. Segal, G., M. Purcell, and H.A. Shuman, Host cell killing and bacterial conjugation require overlapping sets of genes within a 22-kb region of the Legionella pneumophila genome. Proc Natl Acad Sci U S A, 1998. 95(4): p. 1669-74.

118. Segal, G., J.J. Russo, and H.A. Shuman, Relationships between a new type IV secretion system and the icm/dot virulence system of Legionella pneumophila. Mol Microbiol, 1999. 34(4): p. 799-809. 
119. Christie, P.J., Type IV secretion: intercellular transfer of macromolecules by systems ancestrally related to conjugation machines. Mol Microbiol, 2001. 40(2): p. 294-305.

120. Christie, P.J. and J.P. Vogel, Bacterial type IV secretion: conjugation systems adapted to deliver effector molecules to host cells. Trends Microbiol, 2000. 8(8): p. 354-60.

121. Voth, D.E., L.J. Broederdorf, and J.G. Graham, Bacterial Type IV secretion systems: versatile virulence machines. Future Microbiol, 2012. 7(2): p. 241-57.

122. Vincent, C.D., et al., Identification of the core transmembrane complex of the Legionella Dot/Icm type IV secretion system. Mol Microbiol, 2006. 62(5): p. 1278-91.

123. Zhu, W., et al., Comprehensive identification of protein substrates of the Dot/Icm type IV transporter of Legionella pneumophila. PLoS One, 2011. 6(3): p. e17638.

124. Luo, Z.Q. and R.R. Isberg, Multiple substrates of the Legionella pneumophila Dot/Icm system identified by interbacterial protein transfer. Proc Natl Acad Sci U S A, 2004. 101(3): p. 841-6.

125. Ensminger, A.W. and R.R. Isberg, Legionella pneumophila Dot/Icm translocated substrates: a sum of parts. Curr Opin Microbiol, 2009. 12(1): p. 67-73.

126. Hubber, A. and C.R. Roy, Modulation of host cell function by Legionella pneumophila type IV effectors. Annu Rev Cell Dev Biol, 2010. 26: p. 261-83.

127. Ninio, S., et al., The Legionella IcmS-IcmW protein complex is important for Dot/Icm-mediated protein translocation. Mol Microbiol, 2005. 55(3): p. 912-26.

128. Campodonico, E.M., L. Chesnel, and C.R. Roy, A yeast genetic system for the identification and characterization of substrate proteins transferred into host cells by the Legionella pneumophila Dot/Icm system. Mol Microbiol, 2005. 56(4): p. 918-33.

129. Habyarimana, F., et al., Role for the Ankyrin eukaryotic-like genes of Legionella pneumophila in parasitism of protozoan hosts and human macrophages. Environ Microbiol, 2008. 10(6): p. 1460-74.

130. Bardill, J.P., J.L. Miller, and J.P. Vogel, IcmS-dependent translocation of SdeA into macrophages by the Legionella pneumophila type IV secretion system. Mol Microbiol, 2005. 56(1): p. 90-103.

131. Al-Khodor, S., et al., A Dot/Icm-translocated ankyrin protein of Legionella pneumophila is required for intracellular proliferation within human macrophages and protozoa. Mol Microbiol, 2008. 70(4): p. 908-23.

132. Charpentier, X., et al., Chemical genetics reveals bacterial and host cell functions critical for type IV effector translocation by Legionella pneumophila. PLoS Pathog, 2009. 5(7): p. e1000501.

133. Ahuja, N., P. Kumar, and R. Bhatnagar, The adenylate cyclase toxins. Crit Rev Microbiol, 2004. 30(3): p. 187-96.

134. Conover, G.M., et al., The Legionella pneumophila LidA protein: a translocated substrate of the Dot/Icm system associated with maintenance of bacterial integrity. Mol Microbiol, 2003. 48(2): p. 305-21.

135. Price, C.T., et al., Exploitation of conserved eukaryotic host cell farnesylation machinery by an F-box effector of Legionella pneumophila. J Exp Med, 2010. 207(8): p. 1713-26. 
136. Liu, Y. and Z.Q. Luo, The Legionella pneumophila effector SidJ is required for efficient recruitment of endoplasmic reticulum proteins to the bacterial phagosome. Infect Immun, 2007. 75(2): p. 592-603.

137. Cazalet, C., et al., Evidence in the Legionella pneumophila genome for exploitation of host cell functions and high genome plasticity. Nat Genet, 2004. 36(11): p. 1165-73.

138. de Felipe, K.S., et al., Evidence for acquisition of Legionella type IV secretion substrates via interdomain horizontal gene transfer. J Bacteriol, 2005. 187(22): p. 7716-26.

139. Bender, J., et al., Phospholipase PlaB of Legionella pneumophila represents a novel lipase family: protein residues essential for lipolytic activity, substrate specificity, and hemolysis. J Biol Chem, 2009. 284(40): p. 27185-94.

140. Bruggemann, H., C. Cazalet, and C. Buchrieser, Adaptation of Legionella pneumophila to the host environment: role of protein secretion, effectors and eukaryotic-like proteins. Curr Opin Microbiol, 2006. 9(1): p. 86-94.

141. de Felipe, K.S., et al., Legionella eukaryotic-like type IV substrates interfere with organelle trafficking. PLoS Pathog, 2008. 4(8): p. e1000117.

142. Gomez-Valero, L., et al., Comparative and functional genomics of legionella identified eukaryotic like proteins as key players in host-pathogen interactions. Front Microbiol, 2011. 2: p. 208.

143. Machner, M.P. and R.R. Isberg, Targeting of host Rab GTPase function by the intravacuolar pathogen Legionella pneumophila. Dev Cell, 2006. 11(1): p. 47-56.

144. Murata, T., et al., The Legionella pneumophila effector protein DrrA is a Rab1 guanine nucleotide-exchange factor. Nat Cell Biol, 2006. 8(9): p. 971-7.

145. Derre, I. and R.R. Isberg, LidA, a translocated substrate of the Legionella pneumophila type IV secretion system, interferes with the early secretory pathway. Infect Immun, 2005. 73(7): p. 4370-80.

146. Chavrier, P. and B. Goud, The role of ARF and Rab GTPases in membrane transport. Curr Opin Cell Biol, 1999. 11(4): p. 466-75.

147. Derre, I. and R.R. Isberg, Legionella pneumophila replication vacuole formation involves rapid recruitment of proteins of the early secretory system. Infect Immun, 2004. 72(5): p. 3048-53.

148. Creasey, E.A. and R.R. Isberg, The protein SdhA maintains the integrity of the Legionella-containing vacuole. Proc Natl Acad Sci U S A, 2012. 109(9): p. 34816.

149. Banga, S., et al., Legionella pneumophila inhibits macrophage apoptosis by targeting pro-death members of the Bcl2 protein family. Proc Natl Acad Sci U S A, 2007. 104(12): p. 5121-6.

150. Laguna, R.K., et al., A Legionella pneumophila-translocated substrate that is required for growth within macrophages and protection from host cell death. Proc Natl Acad Sci U S A, 2006. 103(49): p. 18745-50.

151. Harding, C.R., et al., The Dot/Icm Effector SdhA Is Necessary for Virulence of Legionella pneumophila in Galleria mellonella and A/J Mice. Infect Immun, 2013. 81(7): p. 2598-605.

152. Zhu, W., et al., Induction of caspase 3 activation by multiple Legionella pneumophila Dot/Icm substrates. Cell Microbiol, 2013. 15(11): p. 1783-95. 
153. Fontana, M.F., et al., Secreted bacterial effectors that inhibit host protein synthesis are critical for induction of the innate immune response to virulent Legionella pneumophila. PLoS Pathog, 2011. 7(2): p. e1001289.

154. Shen, X., et al., Targeting eEF1A by a Legionella pneumophila effector leads to inhibition of protein synthesis and induction of host stress response. Cell Microbiol, 2009. 11(6): p. 911-26.

155. Asrat, S., A.S. Dugan, and R.R. Isberg, The frustrated host response to Legionella pneumophila is bypassed by MyD88-dependent translation of pro-inflammatory cytokines. PLoS Pathog, 2014. 10(7): p. e1004229.

156. Ivanov, S.S. and C.R. Roy, Pathogen signatures activate a ubiquitination pathway that modulates the function of the metabolic checkpoint kinase mTOR. Nat Immunol, 2013. 14(12): p. 1219-28.

157. Cunha, S.R. and P.J. Mohler, Ankyrin protein networks in membrane formation and stabilization. J Cell Mol Med, 2009. 13(11-12): p. 4364-76.

158. Baines, A.J., Evolution of spectrin function in cytoskeletal and membrane networks. Biochem Soc Trans, 2009. 37(Pt 4): p. 796-803.

159. Al-Khodor, S., et al., Functional diversity of ankyrin repeats in microbial proteins. Trends Microbiol, 2010. 18(3): p. 132-9.

160. Mosavi, L.K., et al., The ankyrin repeat as molecular architecture for protein recognition. Protein Sci, 2004. 13(6): p. 1435-48.

161. Blanie, S., et al., $M N F$, an ankyrin repeat protein of myxoma virus, is part of a native cellular SCF complex during viral infection. Virol J, 2010. 7: p. 56.

162. Low, C., et al., Structural insights into an equilibrium folding intermediate of an archaeal ankyrin repeat protein. Proc Natl Acad Sci U S A, 2008. 105(10): p. 3779-84.

163. Bork, P., Hundreds of ankyrin-like repeats in functionally diverse proteins: mobile modules that cross phyla horizontally? Proteins, 1993. 17(4): p. 363-74.

164. Voronin, D.A. and E.V. Kiseleva, [Functional role of proteins containing ankyrin repeats]. Tsitologiia, 2007. 49(12): p. 989-99.

165. Bernal-Quiros, M., et al., BANK1 and BLK act through phospholipase $C$ gamma 2 in B-cell signaling. PLoS One, 2013. 8(3): p. e59842.

166. Dhyani, A., et al., ANKHD1 regulates cell cycle progression and proliferation in multiple myeloma cells. FEBS Lett, 2012. 586(24): p. 4311-8.

167. Kohl, A., et al., Designed to be stable: crystal structure of a consensus ankyrin repeat protein. Proc Natl Acad Sci U S A, 2003. 100(4): p. 1700-5.

168. Binz, H.K., et al., Crystal structure of a consensus-designed ankyrin repeat protein: implications for stability. Proteins, 2006. 65(2): p. 280-4.

169. Becerra, C., et al., Ankyrin repeat-containing proteins in Arabidopsis: characterization of a novel and abundant group of genes coding ankyrintransmembrane proteins. Gene, 2004. 340(1): p. 111-21.

170. Mosavi, L.K., D.L. Minor, Jr., and Z.Y. Peng, Consensus-derived structural determinants of the ankyrin repeat motif. Proc Natl Acad Sci U S A, 2002. 99(25): p. 16029-34.

171. Main, E.R., S.E. Jackson, and L. Regan, The folding and design of repeat proteins: reaching a consensus. Curr Opin Struct Biol, 2003. 13(4): p. 482-9. 
172. Howell, M.L., et al., AnkB, a periplasmic ankyrin-like protein in Pseudomonas aeruginosa, is required for optimal catalase $B($ KatB) activity and resistance to hydrogen peroxide. J Bacteriol, 2000. 182(16): p. 4545-56.

173. Wu, M., et al., Phylogenomics of the reproductive parasite Wolbachia pipientis wMel: a streamlined genome overrun by mobile genetic elements. PLoS Biol, 2004. 2(3): p. E69.

174. Voth, D.E., et al., The Coxiella burnetii ankyrin repeat domain-containing protein family is heterogeneous, with C-terminal truncations that influence Dot/Icmmediated secretion. J Bacteriol, 2009. 191(13): p. 4232-42.

175. Rikihisa, Y. and M. Lin, Anaplasma phagocytophilum and Ehrlichia chaffeensis type IV secretion and Ank proteins. Curr Opin Microbiol, 2010. 13(1): p. 59-66.

176. Habyarimana, F., et al., Molecular characterization of the Dot/Icm-translocated AnkH and AnkJ eukaryotic-like effectors of Legionella pneumophila. Infect Immun, 2010. 78(3): p. 1123-34.

177. Al-Khodor, S., T. Al-Quadan, and Y. Abu Kwaik, Temporal and differential regulation of expression of the eukaryotic-like ankyrin effectors of Legionella pneumophila. Environ Microbiol Rep, 2010. 2(5): p. 677-84.

178. Zhang, F.L. and P.J. Casey, Protein prenylation: molecular mechanisms and functional consequences. Annu Rev Biochem, 1996. 65: p. 241-69.

179. Casey, P.J., Biochemistry of protein prenylation. J Lipid Res, 1992. 33(12): p. 1731-40.

180. Omer, C.A. and J.B. Gibbs, Protein prenylation in eukaryotic microorganisms: genetics, biology and biochemistry. Mol Microbiol, 1994. 11(2): p. 219-25.

181. Gelb, M.H., et al., Therapeutic intervention based on protein prenylation and associated modifications. Nat Chem Biol, 2006. 2(10): p. 518-28.

182. Maltese, W.A., Posttranslational modification of proteins by isoprenoids in mammalian cells. Faseb j, 1990. 4(15): p. 3319-28.

183. Wollack, J.W., et al., Multifunctional prenylated peptides for live cell analysis. J Am Chem Soc, 2009. 131(21): p. 7293-303.

184. Boyartchuk, V.L., M.N. Ashby, and J. Rine, Modulation of Ras and a-factor function by carboxyl-terminal proteolysis. Science, 1997. 275(5307): p. 1796-800.

185. Hrycyna, C.A. and S. Clarke, Modification of eukaryotic signaling proteins by $C$ terminal methylation reactions. Pharmacol Ther, 1993. 59(3): p. 281-300.

186. Epstein, W.W., et al., Quantitation of prenylcysteines by a selective cleavage reaction. Proc Natl Acad Sci U S A, 1991. 88(21): p. 9668-70.

187. Gao, J., J. Liao, and G.Y. Yang, CAAX-box protein, prenylation process and carcinogenesis. Am J Transl Res, 2009. 1(3): p. 312-25.

188. Hancock, J.F., et al., A CAAX or a CAAL motif and a second signal are sufficient for plasma membrane targeting of ras proteins. Embo j, 1991. 10(13): p. 4033-9.

189. Wedegaertner, P.B., P.T. Wilson, and H.R. Bourne, Lipid modifications of trimeric $G$ proteins. J Biol Chem, 1995. 270(2): p. 503-6.

190. Evanko, D.S., M.M. Thiyagarajan, and P.B. Wedegaertner, Interaction with Gbetagamma is required for membrane targeting and palmitoylation of Galpha(s) and Galpha(q). J Biol Chem, 2000. 275(2): p. 1327-36. 
191. Wright, L.P. and M.R. Philips, Thematic review series: lipid posttranslational modifications. CAAX modification and membrane targeting of Ras. J Lipid Res, 2006. 47(5): p. 883-91.

192. Roskoski, R., Jr., Protein prenylation: a pivotal posttranslational process. Biochem Biophys Res Commun, 2003. 303(1): p. 1-7.

193. Tsubaki, M., et al., Nitrogen-containing bisphosphonates induce apoptosis of hematopoietic tumor cells via inhibition of Ras signaling pathways and Bimmediated activation of the intrinsic apoptotic pathway. Biochem Pharmacol, 2013. 85(2): p. 163-72.

194. Appels, N.M., J.H. Beijnen, and J.H. Schellens, Development of farnesyl transferase inhibitors: a review. Oncologist, 2005. 10(8): p. 565-78.

195. Al-Quadan, T., et al., Anchoring of bacterial effectors to host membranes through host-mediated lipidation by prenylation: a common paradigm. Trends Microbiol, 2011. 19(12): p. 573-9.

196. Amaya, M., A. Baranova, and M.L. van Hoek, Protein prenylation: a new mode of host-pathogen interaction. Biochem Biophys Res Commun, 2011. 416(1-2): p. 1-6.

197. Reinicke, A.T., et al., A Salmonella typhimurium effector protein SifA is modified by host cell prenylation and S-acylation machinery. J Biol Chem, 2005. 280(15): p. 14620-7.

198. Boucrot, E., et al., Salmonella typhimurium SifA effector protein requires its membrane-anchoring $C$-terminal hexapeptide for its biological function. J Biol Chem, 2003. 278(16): p. 14196-202.

199. Price, C.T., et al., Host-mediated post-translational prenylation of novel dot/icmtranslocated effectors of legionella pneumophila. Front Microbiol, 2010. 1: p. 131.

200. Ivanov, S.S., et al., Lipidation by the host prenyltransferase machinery facilitates membrane localization of Legionella pneumophila effector proteins. J Biol Chem, 2010. 285(45): p. 34686-98.

201. Grillari, J., R. Grillari-Voglauer, and P. Jansen-Durr, Post-translational modification of cellular proteins by ubiquitin and ubiquitin-like molecules: role in cellular senescence and aging. Adv Exp Med Biol, 2010. 694: p. 172-96.

202. Kerscher, O., R. Felberbaum, and M. Hochstrasser, Modification of proteins by ubiquitin and ubiquitin-like proteins. Annu Rev Cell Dev Biol, 2006. 22: p. 15980.

203. Behrends, C. and J.W. Harper, Constructing and decoding unconventional ubiquitin chains. Nat Struct Mol Biol, 2011. 18(5): p. 520-8.

204. Pickart, C.M., Mechanisms underlying ubiquitination. Annu Rev Biochem, 2001. 70: p. 503-33.

205. Yang, W.L., X. Zhang, and H.K. Lin, Emerging role of Lys-63 ubiquitination in protein kinase and phosphatase activation and cancer development. Oncogene, 2010. 29(32): p. 4493-503.

206. Bhoj, V.G. and Z.J. Chen, Ubiquitylation in innate and adaptive immunity. Nature, 2009. 458(7237): p. 430-7.

207. Mailand, N., et al., RNF8 ubiquitylates histones at DNA double-strand breaks and promotes assembly of repair proteins. Cell, 2007. 131(5): p. 887-900. 
208. Iwai, K., Diverse roles of the ubiquitin system in NF-kappaB activation. Biochim Biophys Acta, 2013.

209. Welchman, R.L., C. Gordon, and R.J. Mayer, Ubiquitin and ubiquitin-like proteins as multifunctional signals. Nat Rev Mol Cell Biol, 2005. 6(8): p. 599609.

210. Walczak, H., K. Iwai, and I. Dikic, Generation and physiological roles of linear ubiquitin chains. BMC Biol, 2012. 10: p. 23.

211. Mukhopadhyay, D. and H. Riezman, Proteasome-independent functions of ubiquitin in endocytosis and signaling. Science, 2007. 315(5809): p. 201-5.

212. Thrower, J.S., et al., Recognition of the polyubiquitin proteolytic signal. Embo j, 2000. 19(1): p. 94-102.

213. Goto, E., et al., Contribution of lysine 11-linked ubiquitination to MIR2-mediated major histocompatibility complex class I internalization. J Biol Chem, 2010. 285(46): p. 35311-9.

214. Schulman, B.A. and J.W. Harper, Ubiquitin-like protein activation by E1 enzymes: the apex for downstream signalling pathways. Nat Rev Mol Cell Biol, 2009. 10(5): p. 319-31.

215. Ye, Y. and M. Rape, Building ubiquitin chains: E2 enzymes at work. Nat Rev Mol Cell Biol, 2009. 10(11): p. 755-64.

216. Ardley, H.C. and P.A. Robinson, E3 ubiquitin ligases. Essays Biochem, 2005. 41: p. $15-30$.

217. Chhangani, D., A.P. Joshi, and A. Mishra, E3 ubiquitin ligases in protein quality control mechanism. Mol Neurobiol, 2012. 45(3): p. 571-85.

218. Jackson, P.K. and A.G. Eldridge, The SCF ubiquitin ligase: an extended look. Mol Cell, 2002. 9(5): p. 923-5.

219. Jackson, P.K., et al., The lore of the RINGs: substrate recognition and catalysis by ubiquitin ligases. Trends Cell Biol, 2000. 10(10): p. 429-39.

220. Zheng, N., et al., Structure of the Cul1-Rbx1-Skp1-F boxSkp2 SCF ubiquitin ligase complex. Nature, 2002. 416(6882): p. 703-9.

221. Skowyra, D., et al., F-box proteins are receptors that recruit phosphorylated substrates to the SCF ubiquitin-ligase complex. Cell, 1997. 91(2): p. 209-19.

222. Bai, C., et al., SKP1 connects cell cycle regulators to the ubiquitin proteolysis machinery through a novel motif, the F-box. Cell, 1996. 86(2): p. 263-74.

223. Price, C.T. and Y.A. Kwaik, Exploitation of Host Polyubiquitination Machinery through Molecular Mimicry by Eukaryotic-Like Bacterial F-Box Effectors. Front Microbiol, 2010. 1: p. 122.

224. Correa, R.L., et al., The Role of F-Box Proteins during Viral Infection. Int J Mol Sci, 2013. 14(2): p. 4030-49.

225. Binder, B.M., et al., The Arabidopsis EIN3 binding F-Box proteins EBF1 and EBF2 have distinct but overlapping roles in ethylene signaling. Plant Cell, 2007. 19(2): p. 509-23.

226. Jin, J., et al., Systematic analysis and nomenclature of mammalian F-box proteins. Genes Dev, 2004. 18(21): p. 2573-80.

227. Gagne, J.M., et al., The F-box subunit of the SCF E3 complex is encoded by a diverse superfamily of genes in Arabidopsis. Proc Natl Acad Sci U S A, 2002. 99(17): p. 11519-24. 
228. Ensminger, A.W. and R.R. Isberg, E3 ubiquitin ligase activity and targeting of BAT3 by multiple Legionella pneumophila translocated substrates. Infect Immun, 2010. 78(9): p. 3905-19.

229. Kubori, T., A. Hyakutake, and H. Nagai, Legionella translocates an E3 ubiquitin ligase that has multiple U-boxes with distinct functions. Mol Microbiol, 2008. 67(6): p. 1307-19.

230. Kubori, T., et al., Legionella metaeffector exploits host proteasome to temporally regulate cognate effector. PLoS Pathog, 2010. 6(12): p. e1001216.

231. Ashida, H., M. Kim, and C. Sasakawa, Exploitation of the host ubiquitin system by human bacterial pathogens. Nat Rev Microbiol, 2014. 12(6): p. 399-413.

232. Zhang, Y., et al., Recognition and ubiquitination of Salmonella type III effector SopA by a ubiquitin E3 ligase, HsRMA1. J Biol Chem, 2005. 280(46): p. 38682-8.

233. Zhang, Y., et al., The inflammation-associated Salmonella SopA is a HECT-like E3 ubiquitin ligase. Mol Microbiol, 2006. 62(3): p. 786-93.

234. Patel, J.C., et al., Diversification of a Salmonella virulence protein function by ubiquitin-dependent differential localization. Cell, 2009. 137(2): p. 283-94.

235. Anderson, D.M. and D.W. Frank, Five mechanisms of manipulation by bacterial effectors: a ubiquitous theme. PLoS Pathog, 2012. 8(8): p. e1002823.

236. Anderson, D.M., et al., Identification of the major ubiquitin-binding domain of the Pseudomonas aeruginosa ExoU A2 phospholipase. J Biol Chem, 2013. 288(37): p. 26741-52.

237. Dorer, M.S., et al., RNA interference analysis of Legionella in Drosophila cells: exploitation of early secretory apparatus dynamics. PLoS Pathog, 2006. 2(4): p. e34.

238. Price, C.T., et al., Molecular mimicry by an F-box effector of Legionella pneumophila hijacks a conserved polyubiquitination machinery within macrophages and protozoa. PLoS Pathog, 2009. 5(12): p. e1000704.

239. Lomma, M., et al., The Legionella pneumophila F-box protein Lpp2082 (AnkB) modulates ubiquitination of the host protein parvin $B$ and promotes intracellular replication. Cell Microbiol, 2010. 12(9): p. 1272-91.

240. Price, C.T., et al., Indispensable role for the eukaryotic-like ankyrin domains of the ankyrin B effector of Legionella pneumophila within macrophages and amoebae. Infect Immun, 2010. 78(5): p. 2079-88.

241. Bruckert, W.M., C.T. Price, and Y. Abu Kwaik, Rapid nutritional remodeling of the host cell upon attachment of Legionella pneumophila. Infect Immun, 2014. 82(1): p. 72-82.

242. Kim, W., et al., Systematic and quantitative assessment of the ubiquitin-modified proteome. Mol Cell, 2011. 44(2): p. 325-40.

243. Rappsilber, J., M. Mann, and Y. Ishihama, Protocol for micro-purification, enrichment, pre-fractionation and storage of peptides for proteomics using StageTips. Nat Protoc, 2007. 2(8): p. 1896-906.

244. Huttlin, E.L., et al., A tissue-specific atlas of mouse protein phosphorylation and expression. Cell, 2010. 143(7): p. 1174-89.

245. Eng, J.K., A.L. McCormack, and J.R. Yates, An approach to correlate tandem mass spectral data of peptides with amino acid sequences in a protein database. $\mathbf{J}$ Am Soc Mass Spectrom, 1994. 5(11): p. 976-89. 
246. Elias, J.E. and S.P. Gygi, Target-decoy search strategy for increased confidence in large-scale protein identifications by mass spectrometry. Nat Methods, 2007. 4(3): p. 207-14.

247. Beausoleil, S.A., et al., A probability-based approach for high-throughput protein phosphorylation analysis and site localization. Nat Biotechnol, 2006. 24(10): p. 1285-92.

248. Ekins, S., et al., Pathway mapping tools for analysis of high content data. Methods Mol Biol, 2007. 356: p. 319-50.

249. van Leeuwen, D.M., et al., Transcriptomic network analysis of micronucleirelated genes: a case study. Mutagenesis, 2011. 26(1): p. 27-32.

250. Price, C.T., et al., Amoeba host-Legionella synchronization of amino acid auxotrophy and its role in bacterial adaptation and pathogenic evolution. Environ Microbiol, 2014. 16(2): p. 350-8.

251. Al-Khodor, S., T. Al-Quadan, and Y. Abu Kwaik, Temporal and differential regulation of expression of the eukaryotic-like ankyrin effectors of $L$. pneumophila. Environ Microbiol Rep, 2010. 2(5): p. 677-684.

252. Molmeret, M., et al., Temporal and spatial trigger of post-exponential virulenceassociated regulatory cascades by Legionella pneumophila after bacterial escape into the host cell cytosol. Environ Microbiol, 2010. 12(3): p. 704-15.

253. Santic, M., et al., Acquisition of the vacuolar ATPase proton pump and phagosome acidification are essential for escape of Francisella tularensis into the macrophage cytosol. Infect Immun, 2008. 76(6): p. 2671-7.

254. Santic, M., et al., A Francisella tularensis pathogenicity island protein essential for bacterial proliferation within the host cell cytosol. Cell Microbiol, 2007. 9(10): p. 2391-403.

255. Al-Quadan, T.P., et al., Anchoring bacterial effectors to host membranes through host-mediated prenylation: A common paradigm. Trends Microbiol, 2011. in press.

256. Price, C.T., et al., Host-mediated post-translational prenylation of novel Dot/Icmtranslocated effectors of Legionella pneumophila. Front Microbio, 2010. 1: p. doi: 10.3389/fmicb.2010.00131.

257. Hubber, A., et al., The machinery at endoplasmic reticulum-plasma membrane contact sites contributes to spatial regulation of multiple Legionella effector proteins. PLoS Pathog, 2014. 10(7): p. e1004222.

258. Nagai, H., et al., A C-terminal translocation signal required for Dot/Icmdependent delivery of the Legionella RalF protein to host cells. Proc Natl Acad Sci U S A, 2005. 102(3): p. 826-31.

259. Hicks, S.W., et al., Subcellular targeting of Salmonella virulence proteins by host-mediated S-palmitoylation. Cell Host Microbe, 2011. 10(1): p. 9-20.

260. Chen, Z. and C.M. Pickart, A 25-kilodalton ubiquitin carrier protein (E2) catalyzes multi-ubiquitin chain synthesis via lysine 48 of ubiquitin. J Biol Chem, 1990. 265(35): p. 21835-42.

261. Hsu, F., et al., The Legionella effector SidC defines a unique family of ubiquitin ligases important for bacterial phagosomal remodeling. Proc Natl Acad Sci U S A, 2014. 111(29): p. 10538-43. 
262. Abu Kwaik, Y. and D. Bumann, Microbial quest for food in vivo: 'nutritional virulence' as an emerging paradigm. Cell Microbiol, 2013. 15(6): p. 882-90.

263. Mills, S.D. and B.B. Finlay, Isolation and characterization of Salmonella typhimurium and Yersinia pseudotuberculosis-containing phagosomes from infected mouse macrophages: Y. pseudotuberculosis traffics to terminal lysosomes where they are degraded. Eur J Cell Biol, 1998. 77(1): p. 35-47.

264. Bruckert, W.M. and Y. Abu Kwaik, The complete and ubiquitinated proteome of the Legionella-containing vacuole within human macrophages. J Proteome Res, 2014.

265. Akhter, A., et al., Caspase-11 promotes the fusion of phagosomes harboring pathogenic bacteria with lysosomes by modulating actin polymerization. Immunity, 2012. 37(1): p. 35-47.

266. Bartke, T., et al., Dual role of BRUCE as an antiapoptotic IAP and a chimeric E2/E3 ubiquitin ligase. Mol Cell, 2004. 14(6): p. 801-11.

267. Zhong, Q., et al., Mule/ARF-BP1, a BH3-only E3 ubiquitin ligase, catalyzes the polyubiquitination of Mcl-1 and regulates apoptosis. Cell, 2005. 121(7): p. 108595.

268. Christensen, D.E., P.S. Brzovic, and R.E. Klevit, E2-BRCA1 RING interactions dictate synthesis of mono- or specific polyubiquitin chain linkages. Nat Struct Mol Biol, 2007. 14(10): p. 941-8.

269. Gudjonsson, T., et al., TRIP12 and UBR5 suppress spreading of chromatin ubiquitylation at damaged chromosomes. Cell, 2012. 150(4): p. 697-709.

270. Han, S., et al., The E3 ubiquitin ligase protein associated with Myc (Pam) regulates mammalian/mechanistic target of rapamycin complex 1 (mTORC1) signaling in vivo through $N$ - and C-terminal domains. J Biol Chem, 2012. 287(36): p. 30063-72.

271. Chen, T., et al., mUbiSiDa: a comprehensive database for protein ubiquitination sites in mammals. PLoS One, 2014. 9(1): p. e85744.

272. Yamamoto, Y., et al., Growth of Legionella pneumophila in thioglycolate-elicited peritoneal macrophages from A/J mice. Infect Immun, 1988. 56(2): p. 370-5.

273. Sgarbanti, M., et al., IRF-7: new role in the regulation of genes involved in adaptive immunity. Ann N Y Acad Sci, 2007. 1095: p. 325-33.

274. Young, J.A., et al., Fas-associated death domain (FADD) and the E3 ubiquitinprotein ligase TRIM21 interact to negatively regulate virus-induced interferon production. J Biol Chem, 2011. 286(8): p. 6521-31.

275. Bannerman, D.D., et al., Divergence of bacterial lipopolysaccharide proapoptotic signaling downstream of IRAK-1. J Biol Chem, 2002. 277(10): p. 804853.

276. Halestrap, A.P., The SLC16 gene family - structure, role and regulation in health and disease. Mol Aspects Med, 2013. 34(2-3): p. 337-49.

277. Kong, S.K. and P.B. Chock, Protein ubiquitination is regulated by phosphorylation. An in vitro study. J Biol Chem, 1992. 267(20): p. 14189-92.

278. Hou, D., et al., Activation-dependent ubiquitination of a $T$ cell antigen receptor subunit on multiple intracellular lysines. J Biol Chem, 1994. 269(19): p. 14244-7. 
279. Zhou, P. and P.M. Howley, Ubiquitination and degradation of the substrate recognition subunits of SCF ubiquitin-protein ligases. Mol Cell, 1998. 2(5): p. 571-80.

280. Galan, J.M. and M. Peter, Ubiquitin-dependent degradation of multiple F-box proteins by an autocatalytic mechanism. Proc Natl Acad Sci U S A, 1999. 96(16): p. 9124-9.

281. Angot, A., et al., Exploitation of eukaryotic ubiquitin signaling pathways by effectors translocated by bacterial type III and type IV secretion systems. PLoS Pathog, 2007. 3(1): p. e3.

282. Kubori, T. and J.E. Galan, Temporal regulation of salmonella virulence effector function by proteasome-dependent protein degradation. Cell, 2003. 115(3): $\mathrm{p}$. $333-42$.

283. Wickliffe, K.E., et al., K11-linked ubiquitin chains as novel regulators of cell division. Trends Cell Biol, 2011. 21(11): p. 656-63.

284. Matsumoto, M.L., et al., K11-linked polyubiquitination in cell cycle control revealed by a K11 linkage-specific antibody. Mol Cell, 2010. 39(3): p. 477-84.

285. Fei, C., et al., Smurfl-mediated Lys29-linked nonproteolytic polyubiquitination of axin negatively regulates Wnt/beta-catenin signaling. Mol Cell Biol, 2013. 33(20): p. 4095-105. 


\section{CURRICULUM VITAE}

\begin{tabular}{|c|c|c|c|}
\hline \multicolumn{2}{|l|}{$\begin{array}{l}\text { NAME } \\
\text { William M. Bruckert }\end{array}$} & \multicolumn{2}{|c|}{$\begin{array}{l}\text { POSITION TITLE } \\
\text { PhD student }\end{array}$} \\
\hline INSTITUTION AND LOCATION & DEGREE & YEAR(S) & FIELD OF STUDY \\
\hline $\begin{array}{l}\text { Middle Tennessee State University. } \\
\text { Murfreesboro, TN }\end{array}$ & B.S. & $2003-2008$ & Biology and Chemistry \\
\hline University of Louisville. Louisville, $\mathrm{KY}$ & M.S. & 2008-2010 & Microbiology \\
\hline University of Louisville. Louisville, KY & Ph.D & 2010-2014 & Microbiology \\
\hline
\end{tabular}

\section{Work Experience}

$\mathrm{Ph}$.D. Graduate Student. University of Louisville 2008-2014. I currently work in the laboratory of Dr. Yousef Abu-Kwaik, studying the Legionella pneumophila translocated effector AnkB.

Undergraduate Research Student. Middle Tennessee State University. I worked in the laboratory of Dr. Mary Farone, studying Legionella-like amoebal pathogens obtained from government water towers.

\section{Presentations}

Midwest Microbial Pathogenesis Conference. August 2013

Poster. Nutritional Preparation of the Host Cell by Extracellular Legionella pneumophila

Research Louisville. September 2013

Poster. Nutritional Preparation of the Host Cell by Extracellular Legionella pneumophila

Midwest Microbial Pathogenesis Conference. September 2014

Poster. The complete and ubiquitinated proteome of the Legionella-containing vacuole within human macrophages

\section{Talks at scientific meetings}

Midwest Microbial Pathogenesis Conference. August 2013

Title. Nutritional Preparation of the Host Cell by Extracellular Legionella pneumophila 


\section{Publications.}

1. Bruckert, W.M., C.T. Price, and Y. Abu Kwaik, Rapid nutritional remodeling of the host cell upon attachment of Legionella pneumophila. Infect Immun, 2014. 82(1): p. $72-$ 82 .

2. Bruckert, W.M. and Y. Abu Kwaik. The complete and ubiquitinated proteome of the Legionella-containing vacuole within human macrophages. Journal of Proteome Research, 2014.

3. Bruckert, W.M. and Y. Abu Kwaik. Lysine ${ }^{11}$-linked polyubiquitination of the AnkB effector of Legionella pneumophila. Submitted 